\title{
Dynamic compressive behaviour of cellular materials: A review of phenomenon, mechanism and modelling
}

DOI:

10.1016/j.ijimpeng.2017.10.006

\section{Document Version}

Accepted author manuscript

Link to publication record in Manchester Research Explorer

\section{Citation for published version (APA):}

Sun, Y., \& Li, Q. M. (2018). Dynamic compressive behaviour of cellular materials: A review of phenomenon, mechanism and modelling. International Journal of Impact Engineering, 112, 74-115.

https://doi.org/10.1016/j.ijimpeng.2017.10.006

\section{Published in:}

International Journal of Impact Engineering

\section{Citing this paper}

Please note that where the full-text provided on Manchester Research Explorer is the Author Accepted Manuscript or Proof version this may differ from the final Published version. If citing, it is advised that you check and use the publisher's definitive version.

\section{General rights}

Copyright and moral rights for the publications made accessible in the Research Explorer are retained by the authors and/or other copyright owners and it is a condition of accessing publications that users recognise and abide by the legal requirements associated with these rights.

\section{Takedown policy}

If you believe that this document breaches copyright please refer to the University of Manchester's Takedown Procedures [http://man.ac.uk/04Y6Bo] or contact uml.scholarlycommunications@manchester.ac.uk providing relevant details, so we can investigate your claim.

\section{OPEN ACCESS}




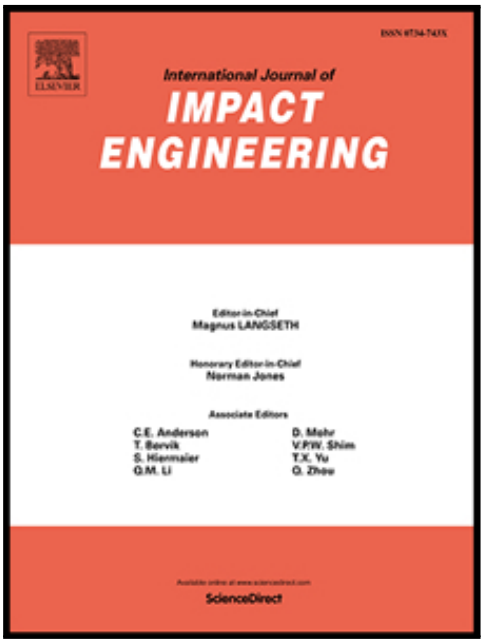

Dynamic compressive behaviour of cellular materials: a review of phenomenon, mechanism and modelling

Yongle Sun, Q.M. Li

PII:

DOI:

Reference:

To appear in:

Received date:

Revised date:

Accepted date:
S0734-743X(17)30166-5

10.1016/j.ijimpeng.2017.10.006

IE 3002

International Journal of Impact Engineering

28 February 2017

9 October 2017

11 October 2017

Please cite this article as: Yongle Sun , Q.M. Li , Dynamic compressive behaviour of cellular materials: a review of phenomenon, mechanism and modelling, International Journal of Impact Engineering (2017), doi: 10.1016/j.ijimpeng.2017.10.006

This is a PDF file of an unedited manuscript that has been accepted for publication. As a service to our customers we are providing this early version of the manuscript. The manuscript will undergo copyediting, typesetting, and review of the resulting proof before it is published in its final form. Please note that during the production process errors may be discovered which could affect the content, and all legal disclaimers that apply to the journal pertain. 


\section{Highlights}

- Dynamic plastic properties, deformation modes, constitutive relations and shock states are described

- Experimental observations in the quasi-static, transitional dynamic and shock regimes are presented

- Mechanisms associated with inertia, enclosed gas and microscopic strain-rate sensitivity of base material are elucidated

- Mesoscopic modelling and its applications are discussed with regard to idealised and realistic cell structures

- Macroscopic continuum-based modelling for compression-dominated Toading is summarised and commented 


\title{
Dynamic compressive behaviour of cellular materials: a review of phenomenon, mechanism and modelling
}

\author{
Yongle $\operatorname{Sun}^{1 \S}$, Q.M. Li ${ }^{1,2 *}$ \\ ${ }^{1}$ School of Mechanical, Aerospace and Civil Engineering, The University of Manchester, \\ Sackville Street, Manchester M13 9PL, UK \\ ${ }^{2}$ State Key Laboratory of Explosion Science and Technology, Beijing Institute of \\ Technology, Beijing 100081, PR China
}

\begin{abstract}
Dynamic compressive behaviour of cellular materials is crucial to their applications in energy absorption, ballistic mitigation and blast/impact protection. The recent research progress in this subject has led to an improved understanding of the experimental, analytical and numerical observations. This review focuses on the aspects of phenomena, mechanisms and modelling on the concerned subject. Attention is paid to linking macroscopic dynamic compressive behaviour with the subscale influential factors. The characteristics of cellular materials at different spatial scales and their compressive behaviours at different loading rates are introduced, based on experimental observations in the quasi-static, transitional dynamic and shock regimes of compression. Then a comprehensive discussion about the roles of the micro- and meso-scale mechanisms in the dynamic compressive behaviour is presented. Finally, important modelling approaches and results are reviewed and commented. The main conclusions are: (1) the strain-rate sensitivity of cellular materials is closely associated with base material properties (both quasi-static and dynamic ones) and cell structure; (2) the compaction shock in cellular materials has mesoscopic structural causes and its formation leads to unique deformation mode, load transmission and stress-strain states; (3) shock initiation requires sufficient loading rate or intensity, and its critical condition can be described based on impact velocity; (4) cell-based modelling is useful for the identification and examination of the underpinning mechanisms, while continuum-based modelling is necessary for the analysis of structures made of cellular materials. Outstanding issues on the subject of the dynamic compressive behaviour of cellular materials are also addressed.
\end{abstract}

Keywords: Foam; Honeycomb; Wood; Strain-rate effect; Shock; Dynamic compressive behaviour; Multiscale analysis; Modelling

\footnotetext{
$\S$ Correspondence: yongle.sun@manchester.ac.uk; sunyongletl@gmail.com

* Corresponding author. Email address: Qingming.Li@manchester.ac.uk (Q.M. Li)
} 


\section{Nomenclature}

\begin{tabular}{|c|c|}
\hline$a$ & Acceleration \\
\hline$d$ & Equivalent diameter of cell \\
\hline$h$ & Current height (or length) of sample \\
\hline$h_{\mathrm{a}}, h_{\mathrm{b}}$ & $\begin{array}{l}\text { Height (or length) of material ahead of and behind shock front, } \\
\text { respectively, in the current configuration }\end{array}$ \\
\hline$l$ & Length of cell edge \\
\hline$p_{0, p} p$ & Initial and current pressure of enclosed gas, respectively \\
\hline$q_{\mathrm{a},} q_{\mathrm{b}}$ & Heat flux density ahead of and behind shock front, respect \\
\hline$t$ & Time \\
\hline$u$ & Displacement \\
\hline$w$ & Thickness of cell wall/strut \\
\hline$D$ & Hardening parameter in dynamic stress-strain relation \\
\hline E & Elastic modulus \\
\hline$E_{\mathrm{p}}$ & Hardening modulus in linear hardening plastic model \\
\hline$H$ & Initial height (or length) of a sample \\
\hline$H_{\mathrm{a}}, H_{\mathrm{b}}$ & $\begin{array}{l}\text { Height (or length) of material ahead of and behind shock front, } \\
\text { respectively, in the original configuration }\end{array}$ \\
\hline$K$ & Hardening parameter in quasi-static stress-strain relation \\
\hline$P, P_{\mathrm{m}}$ & Current and maximum pressure, respectively, during blast loading \\
\hline$T_{\mathrm{m}}$ & Melting temperature $Y$ \\
\hline$T_{0}, T$ & Initial and eurrent temperature, respectively \\
\hline$U_{\mathrm{a}}, U_{\mathrm{b}}$ & $\begin{array}{l}\text { Internal energy density ahead of and behind shock } \\
\text { front, respectively }\end{array}$ \\
\hline \multicolumn{2}{|r|}{ Energy absorption in the plateau stage } \\
\hline$V_{0}$, & and current velocity, respectively \\
\hline & y of material ahead of and behind shock front, respectively \\
\hline$V$ & Impact velocity \\
\hline & Critical impact velocity for shock initiation \\
\hline & Reference velocity in linear Hugoniot relation \\
\hline & Propagation velocity of shock front \\
\hline & Strain \\
\hline$\dot{\varepsilon}^{\prime}$ & Strain-rate \\
\hline$\varepsilon_{\mathrm{a}}, \varepsilon_{\mathrm{b}}$ & Strain ahead of and behind shock front, respectively \\
\hline$\dot{\varepsilon}_{\mathrm{a}-\mathrm{c}}, \dot{\varepsilon}_{\mathrm{i}-\mathrm{c}}$ & Critical strain-rate for an adiabatic process and an isothermal \\
\hline & process of internal gas pressurisation, respectively \\
\hline$\varepsilon_{\mathrm{c} 0}$ & Strain at the onset of cell collapse, when shock is absent \\
\hline
\end{tabular}




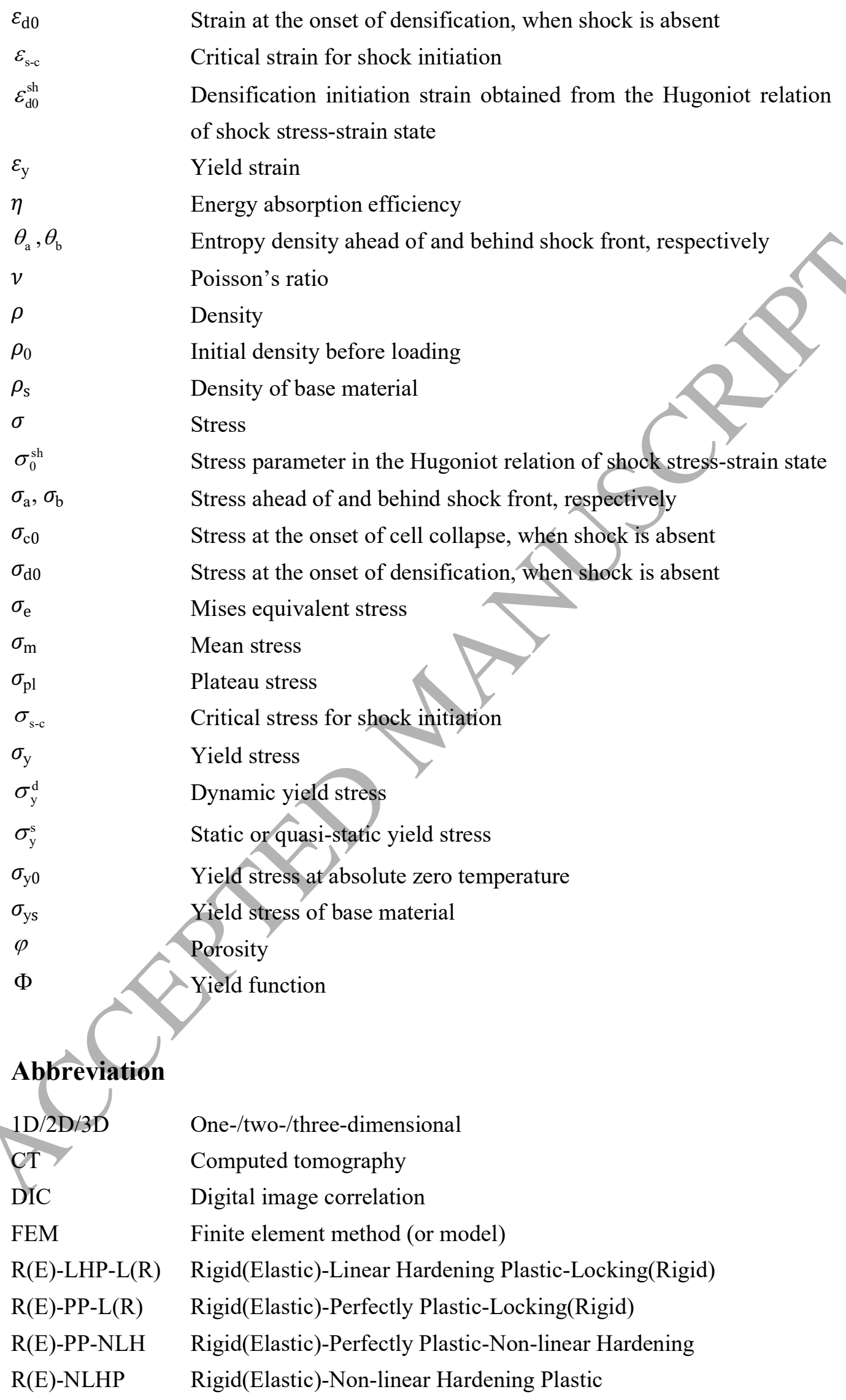




$\begin{array}{ll}\text { R(E)-S-H } & \text { Rigid(Elastic)-Softening-Hardening } \\ \text { RD } & \text { Relative density } \\ \text { RVE } & \text { Representative volume element } \\ \text { SEM } & \text { Scanning electron microscopy } \\ \text { SHPB } & \text { Split Hopkinson pressure bar }\end{array}$

\section{Introduction}

\subsection{Cellular materials at different spatial/temporal scales}

Nature materials often exhibit certain optimised structure and functionality realised by the evolution over millions of years. Many natural materials, such as wood, coral and cancellous bone, are composed of unique cell structures which enable them to possess unusual properties such as ultra-low density and multi-functionality. This enlightens and motivates humans to produce various synthetic materials similar to natural cellular materials in order to meet the continuing demands of weight reduction and function integration in engineering structures. The common feature of cellular materials is the interconnected network of cells to fill the internal space, thereby optimising the load resistance with reduced density. Because of the cell structure, a cellular material can have very high porosity (usually exceeding $70 \%$ and even as high as $99.7 \%$ [1]) and thus much lower density than that of the base material. Cellular materials are also distinct from porous materials which are composed of isolated pores and have relatively low porosity $(<70 \%)$ [1]. Cellular materials can be categorised according to their cell morphology and topology. One widely accepted classification is based on the cell connection, i.e. cells are open or closed, or have semi-openness. Another classification is based on the way in which cells are arranged. For instance, 2D cellular materials (e.g. honeycombs) have cells parallelly arrayed in one direction (i.e. out-of-plane direction), while $3 \mathrm{D}$ céllular materials (e.g. foams) have cells connecting each other by sharing struts (i.e. edges of open cells) or walls (i.e. faces and edges of closed cells) in the whole space occupied.

Cellular materials are attractive for many engineering applications with regard to their mechanical, thermal, acoustic and electromagnetic properties, among which the applications for energy absorption and load attenuation have been continuously increased in transport, aerospace, defence, building and biomedical industries [1-11]. When they are used to absorb energy or mitigate impact/blast loads, their compressive properties, under both quasi-static and dynamic conditions, are of particular importance. In addition, compression tests are used most widely to determine the yield surface and its strain-rate sensitivity in constitutive models 
for cellular materials. The research efforts on the topic of the compressive behaviour of cellular materials, especially those made in the past twenty years, have led to a body of good knowledge for engineering analysis and design. This review summarises and comments on the recent research progress in the dynamic compressive behaviour of cellular materials.

A cellular material consists of at least two phases (i.e. a solid and a gas) and its structural characteristics are manifested at different spatial scales. At least four spatial scales can be distinguished in a cellular material, as shown in Fig. 1. At the macro-scale, the cellular material is characterised by its overall geometrical and physical properties, and the continuum assumption is normally adopted. A macroscopic description of a cellular material implies that a sufficient number of cells are involved. In most cases, a spatial scale on or above the order of $10^{-2} \mathrm{~m}$ can be regarded as macro-scale, but the lower bound may vary in a wide length range depending on cell size (e.g. equivalent diameter, $d$, of cell). The meso-scale defined here is the spatial scale (typically $10^{-4}-10^{-2} \mathrm{~m}$ ) at which the individual cells, which are constructed by the base material and the enclosed gas, are arranged and connected in space. With further reducing the dimension, the micro-scale typically covering $10^{-6}-10^{-4} \mathrm{~m}$ describes how the base material itself is constituted. The nano-scale $\left(<10^{-6} \mathrm{~m}\right)$ may be important when the cell size is extremely small or the base material itself is highly porous, but the nanoscopic behaviour is less frequently encountered in the current engineering applications, and thus, will not be discussed here.

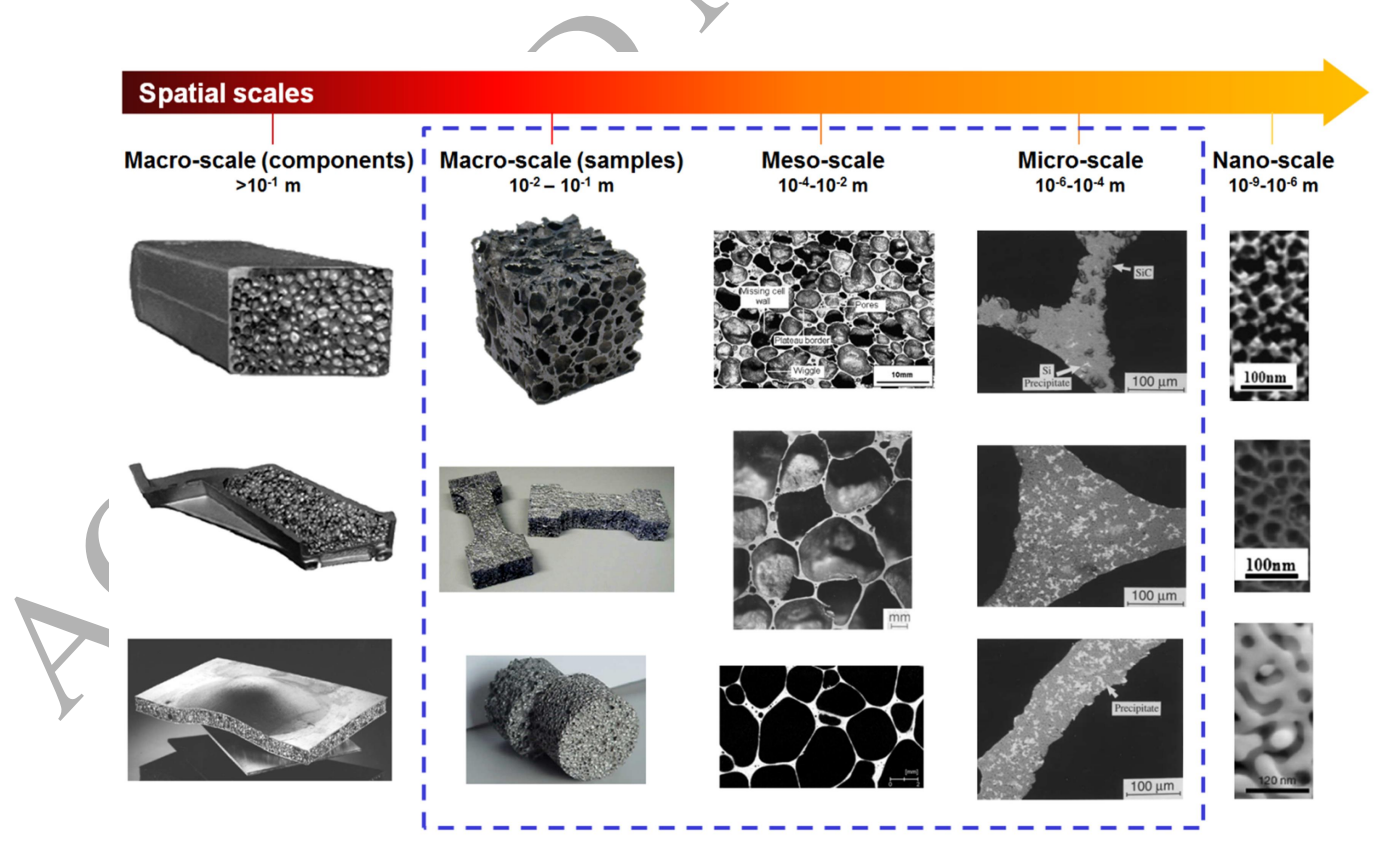

Fig. 1. Structural features of cellular materials (metal foams taken as an example) at different spatial scales. Illustration images are adapted from Refs. [4, 5, 12-17]. 
To guide material optimisation and engineering analysis, the micro- and meso-scale mechanisms controlling the macroscopic material properties have to be understood. Fig. 2 demonstrates the distinctive characteristics and properties of a cellular material at different spatial scales. The base material may exhibit significant micro-structural heterogeneity, but normally it needs to be homogenised to obtain the "bulk" base material properties in order to simplify the material modelling at the meso-scale. The mesoscopic morphology and topology of cells, and their deformation modes, as well as the homogenised properties of the base material and enclosed gas, play important roles in determining the macroscopic material properties (e.g. plastic strength and its strain-rate sensitivity).

The links between the mechanical behaviours at different spatial scales are illustrated in Fig. 2. The multiscale behaviour of a cellular material can be realised from the deformations undergone by the cellular material, individual cell and base material. A macroscopic global strain (or nominal strain) is defined with respect to the gauge length over the representative volume element (RVE) (or a whole sample in a material test), while a macroscopic local strain is equivalent to the mesoscopic global strain defined as a measure of the average deformation experienced by each cell (or a row of cells if one-dimensional simplification is adopted). Such a conceptual equivalence between the macroscopic local strain and mesoscopic global strain for cellular materials has been applied in experimental strain mapping using digital image correlation $[18,19]$, and in numerical strain calculations based on the cross-sectional displacement $[20,21]$ and finite element nodal distance in a cell neighbourhood $[22,23]$. Similarly, the mesoscopic local strain and the microscopic global strain are equivalent, and they are both a measure of the deformation of the base material [21, 24-26]. This conceptual equivalence, again, acts as the link between the meso-scale and micro-scale. When the deformation of an individual phase or grain in the base material is examined, the microscopic local strain measure should be resorted to, which is beyond the scope of this review. It should be noted that the strain or strain-rate at each spatial scale, as discussed throughout this review, refers to the global one averaged over the whole gauge length at that scale, unless otherwise stated. Furthermore, engineering strain measure is adopted. If no specification of the spatial scale is made, macroscopic strain or strain-rate is implied. Similar convention is applied to stress. 


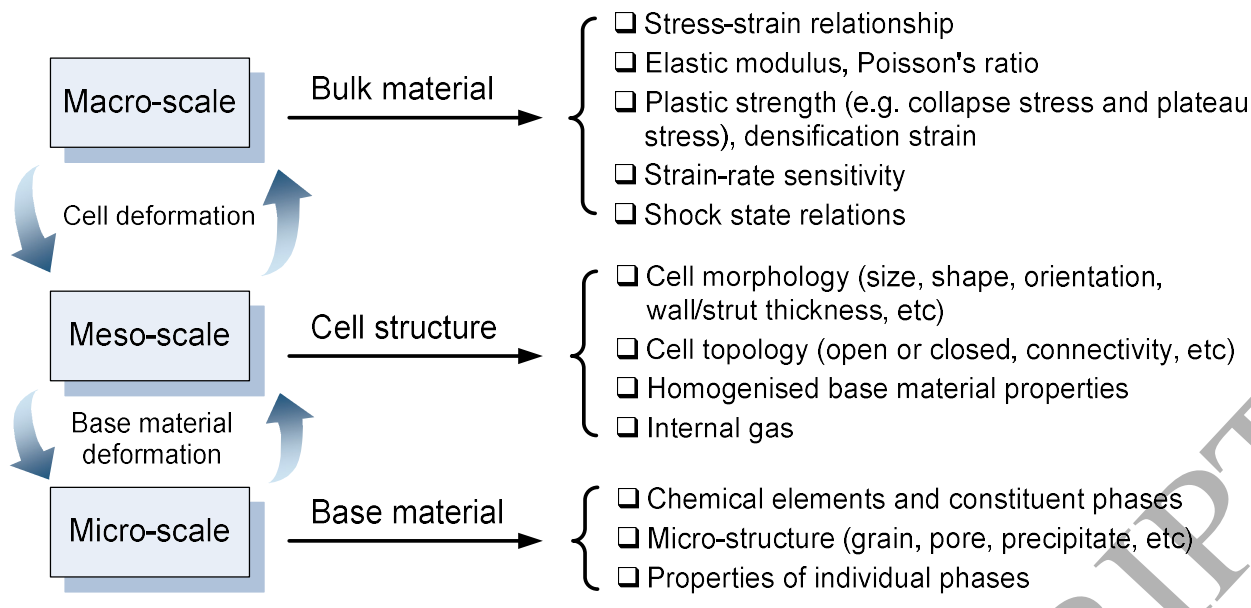

Fig. 2. Characteristics and properties of cellular materials at different spatial scales and their links.

Mesoscopic structural parameters are necessary to quantify the cell characteristics which distinguish cellular materials from other engineering materials. The most important structural parameter is relative density [1], which indicates the proportion of solid content in the cell structure and is expressed as

$$
\mathrm{RD}=\frac{\rho}{\rho_{\mathrm{s}}}=1-\varphi
$$

where $\rho$ is the bulk density, $\rho_{\mathrm{s}}$ is the density of the base material, and $\varphi$ is the porosity. The relative density $\rho / \rho_{\mathrm{s}}$ describes the original cell structure of a cellular material, unless otherwise stated. The bulk density can be measured by the total mass of a specimen divided by the bulk volume. The density of the base material can be estimated by a rule-of-mixtures method if the chemical composition or constituent phases are known. The relative density also can be obtained using geometrical parameters of cells. For example, the relative density of a honeycomb with/regular hexagonal cells can be derived as

$$
\frac{\rho}{\rho_{\mathrm{s}}}=\frac{2}{\sqrt{3}} \frac{w}{l}\left(1-\frac{1}{2 \sqrt{3}} \frac{w}{l}\right)
$$

where $w$ and $l$ are the thickness and length of cell wall, respectively. Formulae to predict or estimate relative densities of cellular materials are given by Gibson and Ashby [1]. Compared to porous materials, cellular materials contain many interconnected cells rather than isolated pores, and thus have much lower relative density. There exists a transition from cellular materials to porous materials when the relative density exceeds approximately $30 \%$ [1].

Other important mesoscopic structural parameters include the dimension, curvature, 
corrugation, orientation, anisotropy and connectivity of the cells or their struts/walls, and so on. Gibson and Ashby [1,27] systematically analysed and demonstrated the correlations between bulk properties and relative density of cellular materials (mainly honeycombs and foams). In the subsequent studies, it has been shown that the morphology [28-35], topology [36-39] and defects [40-46] of cell structure can also significantly affect the bulk properties.

When different temporal scales are considered, the macroscopic compression of cellular materials can be divided into three regimes in terms of the macroscopic loading rate (strain-rate or loading speed), i.e. quasi-static, transitional dynamic and shock regimes, as shown in Fig. 3. Note that the compressive behaviour at very high velocity $(>1000 \mathrm{~m} / \mathrm{s})$ is not included here. Also note that, despite the widely observed shock compression in the vast majority of cellular materials subjected to high speed impact (Sections 2.3.1 and 3.2.1), exception has been reported for dynamic out-of-plane compression of aluminium honeycombs, for which shock effect has not been observed in experiments with an impact speed up to 45 $\mathrm{m} / \mathrm{s}$ [13, 47, 48], nor in cell-based numerical simulations with an impact speed up to $200 \mathrm{~m} / \mathrm{s}$ [49]. Karagiozova and Alves [49] thus stated that "the honeycombs out-of-plane dynamic compression does not pertain to the shock wave phenomenon". However, it should be noted that honeycombs only have one cell in the out-of-plane direction, unlike other cellular materials such as foam and wood. Honeycombs resemble parallelly arrayed tubes, for which the dynamic load transmission during axial high-speed impact is controlled by the stress wave propagation in the tube walls $[50,51]$ rather than cell-wall interaction (e.g. contact and compaction) in foams $[52,53]$. The mesoscopic mechanism of load transmission in cellular materials during high speed impact is still an open topic. In the subsequent discussion on shock, the dynamic out-of-plane compression of honeycombs will be excluded.

In the quasi-static and transitional dynamic regimes, a cellular material is compressed under macroscopic force equilibrium (or macro-inertia effect is insignificant) and the nominal strain-rate (i.e. loading speed divided by sample gauge length) is commonly used as a measure of the overall deformation rate for the description of constitutive behaviour (see Section 2.2). However, in the shock regime the force balance is violated due to the macro-inertial force in axial direction, and the shock propagation leads to discontinuity of macroscopic physical quantities. Consequently, the loading speed, which is correlated with particle velocities within the cellular material, is more appropriate to characterise the compression process [54, 55]. Although not recommended, the nominal strain-rate is occasionally used even when shock occurs, for the convenience of comparison between different compression regimes, but in such a case, the loading speed must be also given for 
the specific length of the sample tested, since the shock behaviour essentially depends on loading speed rather than nominal strain-rate.

Equally importantly, the microscopic strain-rate experienced by the base material can be significantly different from the macroscopic strain-rate experienced by the cellular material, due to nonuniform deformation (e.g. shear bands and crush bands) in the cell structure. Localised deformation is common in cellular materials (see Section 3.2.1). In quasi-static and transitional dynamic regimes, localised large deformation can be correlated with a stress plateau in the stress-strain curve (see Sections 2.1, 2.2 and 3.2.1). However, relatively uniform large deformation could be also produced if the cellular material/e.g. composite metal foam $[56,57])$ has certain mesoscopic stabilising mechanism. In the shock regime, localised and progressive crushing initiates from loading end, and the stress in the crushed zone is significantly larger than that in the uncrushed zone, due to the strong inertia effect (see Sections 2.3 and 3.2.1). Despite the recognised deformation localisation in many cellular materials, the nominal strain and stress are commonly used to characterise the compressive properties. This is acceptable in quasi-static and transitional dynamic regimes, since the internal force equilibrium (or the continuity of macroscopic stress) is established and the localised deformation is randomly distributed in the sample, so that the homogenised stress and strain can be used for engineering analysis. By contrast, the local stresses and strains in the crushed and uncrushed zones, separated by shock front, should be used in the shock regime, and further discussion about this issue will be presented in Sections 2.3, 3.2.1, 4.1.2 and 4.2.1.

The dynamic compression of cellular materials may also involve micro-inertia apart from macro-inertia, which must be distinguished when evaluating the inertia effects. Micro-inertia arises from the acceleration of actual material points in individual cell wall or strut, which is associated with cell deformation (particularly for Type-II structure deformation, as discussed in Section 3.1, see Fig. 15 and Ref. [58]). Macro-inertia in axial direction appears when the axial acceleration of a macroscopic point (e.g. mass centre of a representative volume) of a cellular material is not zero. The evaluation of macro-inertia is based on the macroscopic deformation mode, and the macro-inertia can be represented by the averaged macroscopic density. However, a uniaxial compression of a cellular material actually produces mixed deformation modes (e.g. bending and stretch/contraction) of the base material in the cell structure, potentially leading to amplified transverse micro-inertial force that may affect the deformation process and the axial load resistance of the cellular material. If we simply refer the micro-inertia to the transverse inertia $[26,59]$, the conventional continuum mechanics 
approach is usually not able to capture the micro-inertia effect, partially because most of cellular materials have small macroscopic plastic Poisson's ratio (close to zero [60-62]) but the walls/struts inside the cell structure actually undergo significant transverse motion (lack of constraint due to high porosity) under macroscopic compression. As micro-inertia is sensitive to cell structural characteristics, the evaluation of its effect must consider the cell deformation at the meso-scale. The dynamic compressive properties of a cellular material might change due to micro-inertia effect $[48,63]$, even when macro-inertia has negligible contribution to the compressive resistance of the cellular material.

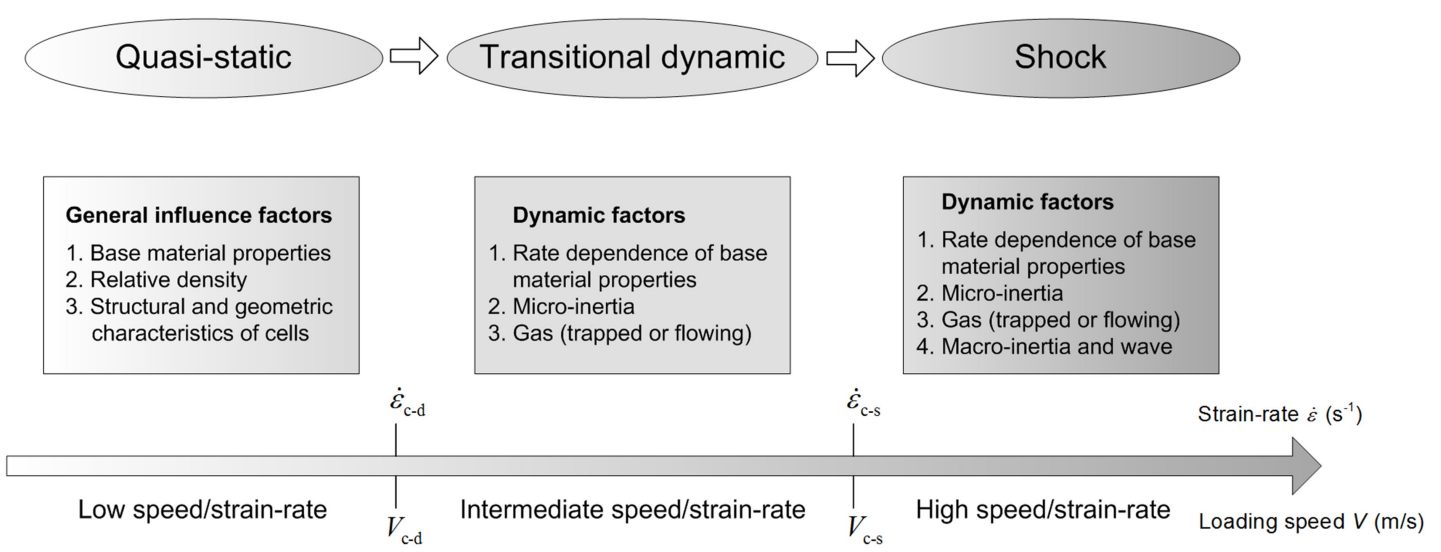

Fig. 3. Compression regimes at different loading rates (strain-rates or loading speeds).

The quasi-static compressive behaviour is normally regarded as the reference case when evaluating the effects of dynamic factors at intermediate and high loading rates. It is generally agreed $[1,64,65]$ that the strain-rate sensitivity of base material, micro-inertia, internal gas, as well as macro-inertia and structural shock wave, are key factors to affect dynamic compressive behaviour. It has been also recognised that distinctive cell deformations can occur in different compression regimes [54, 66-69]. In particular, when shock initiates, the cell deformation is dominated by the propagation of a macroscopically planar discontinuity wave front, which is distinct from the structure-sensitive cell deformation in the quasi-static and transitional dynamic regimes. It may be expected that critical conditions exist between different compression regimes. However, such conditions may not be easily identified if there is no distinct boundary between two considered regimes. For instance, the quasi-static compression is broadly similar to the transitional dynamic compression with regard to cell deformation, due to the lack of macro-inertia effect. By contrast, the shock leads to unique cell deformation and distinctive shock stress-strain states, and hence it is more feasible to 
identify the critical condition for shock initiation.

Microscopic and mesoscopic models are important for identifying mechanisms and quantifying the effects of different small-scale factors. Moreover, exactly the same samples can be used in compression simulations for quasi-static, transitional dynamic and shock regimes, thereby avoiding the uncertainty caused by mesoscopic structural difference between nominal "same" samples used in experiments (this is particularly an issue for stochastic cellular materials with large cells). On the other hand, a macroscopic continuum-based model is needed for structural analysis and design. Such homogenised models represent the averaged responses of a collection of mesoscopic structures of a cellular material, instead of their individual responses in a mesoscopic model. However, it is preferable/ that the continuum-based models are underpinned by the understanding of the micro-and meso-scale mechanisms, rather than merely empirical or phenomenological. The/mechanism-based understanding provides robustness to the predictive capability of analytical and numerical models, which motivates the authors to present a review with the balance between observations, mechanisms and modelling.

\subsection{Objective, scope and outline}

This paper is aimed to review the observations, mechanisms and modelling reported in the literature and furthermore to present a consolidated understanding of the dynamic compressive behaviour of cellular materials. The strain-rate sensitivity of cellular materials has caused much controversy and confusion in the past $[54,55,59,70,71]$. The supposed load attenuation function of cellular materials is also questionable in high speed impact or intensive blast loading [72-77]. Recent progress in research has led to a clearer picture and a better understanding of the experimental and numerical observations. In this review, the dynamic mechanisms operating at the micro- and meso-scale are elucidated on the basis of experimental evidence and cell-based modelling results. The summary of modelling methods is focused on/dynamic compression problems while, as an extension, macroscopic constitutive modelling for isotropic cellular materials subjected to multiaxial or combined loading is also discussed.

The cellular materials examined here are mainly mesoscopic bending-dominated or buckling-prone materials, including foams, honeycombs, wood and other idealised virtual cellular solids (e.g. Kelvin and Voronoi foams), which are used primarily for the purpose of energy absorption and load attenuation. Mesoscopic stretch-dominated cellular materials (e.g. architected lattices) possess better initial stiffness and strength, and their macroscopic properties can be correlated with relative density linearly, unlike the nonlinear correlation of 
bending-dominated cellular materials $[36,37,78,79]$. Stretch-dominated cellular materials are preferable in lightweight structure construction wherein weight reduction and load bearing are most important. However, they are less suitable for energy absorption or load attenuation, as they can be susceptible to significant post-yield softening which leads to a compressive stress-strain curve with high peak stress but much lower plateau stress [39, 78, 80]. Despite the difference in the initial compressive response, at large compressive strains the bendingand stretch-dominated cellular materials actually exhibit similar mesoscopic deformation mode (e.g. buckling collapse and crushing). Shock can also occur in both bending- and stretch-dominated cellular materials, controlled by similar mesoscopic mechanism [81]. Therefore, most of the discussion and conclusions about large-deformation compressive behaviour and the modelling methods presented in this review are also applicable to stretch-dominated cellular materials, despite that the results and analyses of bending-dominated cellular materials are mainly used to obtain the conclusions.

Exceptional compressive behaviours associated with functionally graded [82-84] and hierarchical [85-87] cellular materials, which involve tailored architectures, are not discussed here, and neither is the hypervelocity compressive behaviour $[9,10]$. Large deformation plastic behaviour in compression-dominated loading (mainly dynamic uniaxial compression, with reference to quasi-static case; multiaxial compression or combined loading is briefly discussed in Section 4.2.2) is of major interest here, which is most important for energy absorption and load attenuation, although the elastic properties of cellular materials can be also sensitive to strain-rate [88].

This review paper is divided into five sections, which are devoted to general background (Section 1), experimental observations of macroscopic compressive behaviour (Section 2), micro- and meso-scale examination of mechanisms (Section 3), methodology and application of modelling (Section 4), and concluding remarks (Section 5).

\section{Macroscopic compressive behaviour}

\subsection{Quasi-static regime}

\subsubsection{Stress-strain relation}

Fig. 4 shows typical stress-strain curves of metallic foam, honeycomb and packed hollow sphere structure under quasi-static compression [13]. The tested samples in Fig. 4 have a sufficient number of cells, which avoid sample size effect [89-91], and thus they are representative for the measurement of macroscopic mechanical properties. The compression 
exhibits three distinctive stages: (1) the initial stage wherein stress increases approximately linearly with strain until reaching a local maximum; (2) the subsequent plateau stage wherein stress remains relatively constant over a large strain range (note that it is this unique plateau stage that differentiates cellular solids from porous and fully dense solids); (3) the final densification stage wherein the stress rises rapidly. It should be noted that appreciable stress oscillation may occur in the plateau stage (Fig. 4), which indicates unstable mesoscopic deformations associated with base material properties and cell structure. Similar stress-strain relations also exist for wood [92], polymer foams [93, 94], ceramic foams [95], concrete foams $[96,97]$, and so on.

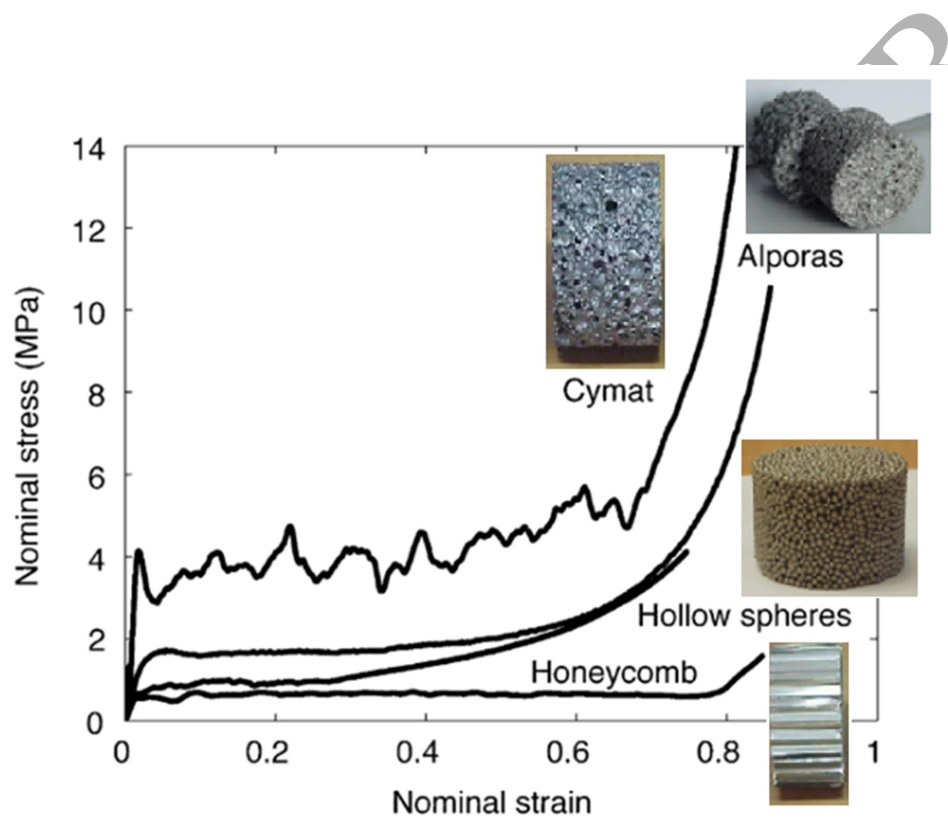

Fig. 4. Quasi-static compressive stress-strain curves of closed-cell aluminium Cymat foam $\left(235 \mathrm{~kg} / \mathrm{m}^{3}\right)$ and Alporas foam $\left(245 \mathrm{~kg} / \mathrm{m}^{3}\right)$, aluminium honeycomb $\left(38 \mathrm{~kg} / \mathrm{m}^{3}\right.$, out-of-plane compression) and packed nickel hollow spheres $\left(219 \mathrm{~kg} / \mathrm{m}^{3}\right)$. [adapted from Ref. 13]

\subsubsection{Plastic properties}

In the initial compression stage, the collapse initiation stress, $\sigma_{\mathrm{c} 0}$, is usually used to indicate the yield strength, which can be obtained as the first peak stress or the stress at which the tangent modulus is the first minimum. Although an offset yield stress (e.g. $0.3 \%$ offset yield point [60]) can be also used to determine the initial yield strength of a cellular material, it requires the knowledge of elastic modulus which may vary with strain and needs considerable extra effort to obtain accurate measurement $[96,98,99]$.

The collapse initiation strain, $\varepsilon_{\mathrm{c} 0}$, can be unambiguously identified as the strain 
corresponding to $\sigma_{\mathrm{c} 0}$, as shown in Fig. 5, indicating the start of the plateau stage. By contrast, a variety of methods have been proposed to determine the densification initiation strain $\varepsilon_{\mathrm{d} 0}$ [100]. Tan et al. [101] first proposed an energy absorption efficiency method, based on the stress-strain curve obtained in a quasi-static compression test, to determine the strain at the onset of densification. Subsequently, Li et al. [100] experimentally investigated the compressive stress-strain curves of various foams using different methods to determine $\varepsilon_{\mathrm{d} 0}$ and concluded that the energy absorption efficiency method is advantageous as it can give unique and consistent results.

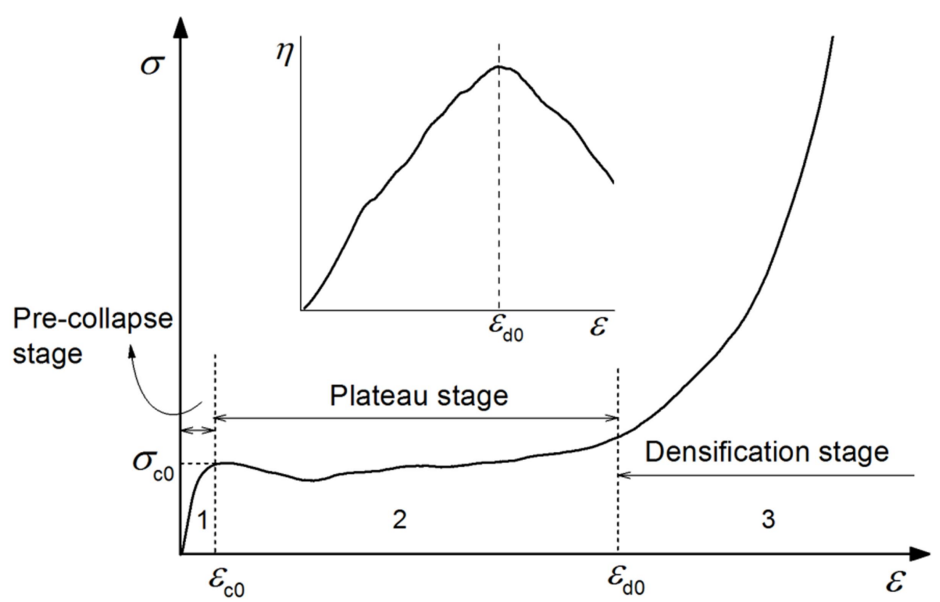

Fig. 5. Schematic diagram of stress-strain curve illustrating the three compression stages (inset showing $\eta-\varepsilon$ curve) [96].)

The energy absorption efficiency is defined as

$$
\eta(\varepsilon)=\frac{1}{\sigma(\varepsilon)} \int_{0}^{\varepsilon} \sigma(\varepsilon) \mathrm{d} \varepsilon
$$

where $\sigma$ and $\varepsilon$ are the nominal compressive stress and strain, respectively. The densification stage begins (or the plateau stage ends), when $\eta$ reaches the maximum, i.e.

$$
\left.\frac{\mathrm{d} \eta(\varepsilon)}{\mathrm{d} \varepsilon}\right|_{\varepsilon=\varepsilon_{\mathrm{d} 0}}=0
$$

where $\varepsilon_{\mathrm{d} 0}$ is the densification initiation strain (Fig. 5). This definition of densification initiation strain has been widely accepted and increasingly used by researchers for various types of cellular materials $[96,102-106]$. It is also noteworthy that the densification initiation 
strain can be derived if the stress-strain equation is known. For instance, when a power law, e.g. $\sigma=\sigma_{\mathrm{c} 0}+k \varepsilon^{n}$, is adopted, we have

$$
\varepsilon_{\mathrm{d} 0}=\left\{\frac{(n+1) \sigma_{\mathrm{c} 0}}{2 k}\left[(n-2)-\sqrt{(n-2)^{2}-\frac{4}{n+1}}\right]\right\}^{1 / n}
$$

The valid condition of the above equation is that the exponent $n$ is not smaller than three, which suggests that the energy absorption efficiency method is applicable only when $n$ has relatively large values. For most of cellular materials (e.g. foams and honeycombs, as shown in Fig. 4), the stress-strain curve has an overall increasing slope and a rapid change of the slope from the plateau stage to the densification stage. Ideally, the slope change is abrupt, e.g. from a very small value to a large value. This justifies the use of a single parameter, i.e. densification initiation strain, to characterise the boundary (or transition) between the plateau stage and densification stage.

In the literature, another term "densification strain" is álso widely used. In most cases, the "densification strain" has the same meaning as the "densification initiation strain", particularly when it is used in an analytical model based on a simplified stress-strain relation [100]. However, the "densification strain" was originally defined by Gibson and Ashby [1] to indicate a limiting strain at which "the opposing walls of the cells crush together and the cell wall material itself is compressed". In other words, the "densification strain" indicates the completion of densification. However, in the practical application of cellular materials and the related engineering design, the onset of densification is more important than the completion of densification. Therefore, only the "densification initiation strain" is discussed in this review.

Densification initiation strain is an important parameter in analytical models wherein the compressive responses in the plateau stage and the densification stage are treated separately (see examples in Section 4.2.1). It is also used as a material parameter to characterise the crushability of a cellular material in terms of the maximum compressive deformation attainable in the plateau stage, in both quasi-static and dynamic cases $[34,69,92,104,107$, 108]. In addition, this parameter is usually required for the determination of the average stress in the plateau stage, i.e. plateau stress, as well as the energy absorption capacity of a cellular material.

The plateau stress can be obtained by the energy equivalence in the plateau stage, viz.

$$
\sigma_{\mathrm{pl}}=\frac{\int_{\varepsilon_{\mathrm{c} 0}}^{\varepsilon_{\mathrm{d} 0}} \sigma(\varepsilon) \mathrm{d} \varepsilon}{\varepsilon_{\mathrm{d} 0}-\varepsilon_{\mathrm{c} 0}}
$$


The calculation of energy absorption normally neglects the pre-collapse and densification stages, as well as elastic energy. Therefore, the plastic energy can be obtained by

$$
U_{\mathrm{pl}}=\int_{\varepsilon_{\mathrm{c} 0}}^{\varepsilon_{\mathrm{d} 0}} \sigma(\varepsilon) \mathrm{d} \varepsilon=\sigma_{\mathrm{pl}}\left(\varepsilon_{\mathrm{d} 0}-\varepsilon_{\mathrm{c} 0}\right)
$$

\subsection{Transitional dynamic regime}

\subsubsection{Strain-rate effect on stress-strain relation}

In the transitional dynamic regime, a macroscopic force balance is established in the cellular material throughout the compression. Standard test methods for intermediate strain-rates, e.g. split Hopkinson pressure bar (SHPB) technique, are widely used to determine dynamic constitutive stress-strain relations of materials. However, the standard SHPB method has several remarkable drawbacks when it is applied to cellular materials. First, the pressure bar has limited diameter but the tested sample must be large enough to avoid size effect [89]; this issue causes either experimental difficulty associated with the requirement of large diameter pressure bars or data scattering when the pressure bar diameter is insufficient $[54,65$, $109,110]$. Second, the wave impedance of the tested sample is usually too low to produce strong signals for metallic pressure bars, and consequently, additional difficulty has to be overcome when low-impedance polymer or hollow metallic pressure bars are used to ensure measurement accuracy [54, 65, 110-113]. Third, the interest in the dynamic crushing stress throughout the plateau stage implies that large deformation of the tested cellular material should be produced by the pressure bar, which requires that the striking bar and the output pressure bar must be sufficiently long to avoid multiple loading-unloading [111]. Fourth, some test setups are incapable of acquiring sufficient information to verify the force equilibrium assumed in a SHPB test for the transitional dynamic regime [54, 65, 109, 114]. Therefore, it is suggested that the SHPB test results for cellular materials should be interpreted with caution, and the experimental uncertainty should be addressed. Other prevalent test methods, such as pendulum and drop hammer, generally have more limitations than SHPB $[58,115,116]$. Recently, some experiment-based inverse methods using digital image correlation technique have been applied to obtain the stress-strain relation within a foam sample, e.g. Lagrangian analysis method [109, 117] and non-parametric analysis method [118], which may overcome the limitation of traditional SHPB method.

On the other hand, Deshpande and Fleck [64] pointed out that cellular materials like aluminium foams are highly heterogeneous and have significant scatters of compressive 
properties, and thus the tested samples can be regarded as strain-rate sensitive only when the increase in dynamic strength is larger than the scatter band (20\% adopted in their work). Indeed, great scatters of compressive properties are widely observed in both quasi-static and dynamic compression tests $[119,120]$, and therefore, sufficient tests should be done to show both the average and the standard deviation at various strain-rates.

The aforementioned experimental uncertainties should be considered when making conclusions from some experimental test results. Nevertheless, most of the reported experimental observations have confirmed that the compressive strength of closed-cell aluminium Alporas foam is sensitive to strain-rate and thus this specific foam is selected as an example here to demonstrate the strain-rate effect. The mechanisms responsible for the rate dependence of Alporas foam will be discussed in Section 3.

Shen et al. [107] used a High Rate Instron Test System to achieve a constant strain-rate up to $220 \mathrm{~s}^{-1}$ in compression tests, and the stress-strain curyes of Alporas foam at different strain-rates are shown in Fig. 6. These results are particularly valuable to examine the strain-rate effect on the whole stress-strain relation, while other dynamic compression tests such as those using SHPB and pendulum normally have difficulty to attain the densification stage due to the shorter loading pulse. From Fig. 6, it is clearly seen that the compressive stress is enhanced and the densification initiation strain is somewhat reduced when the strain-rate increases.

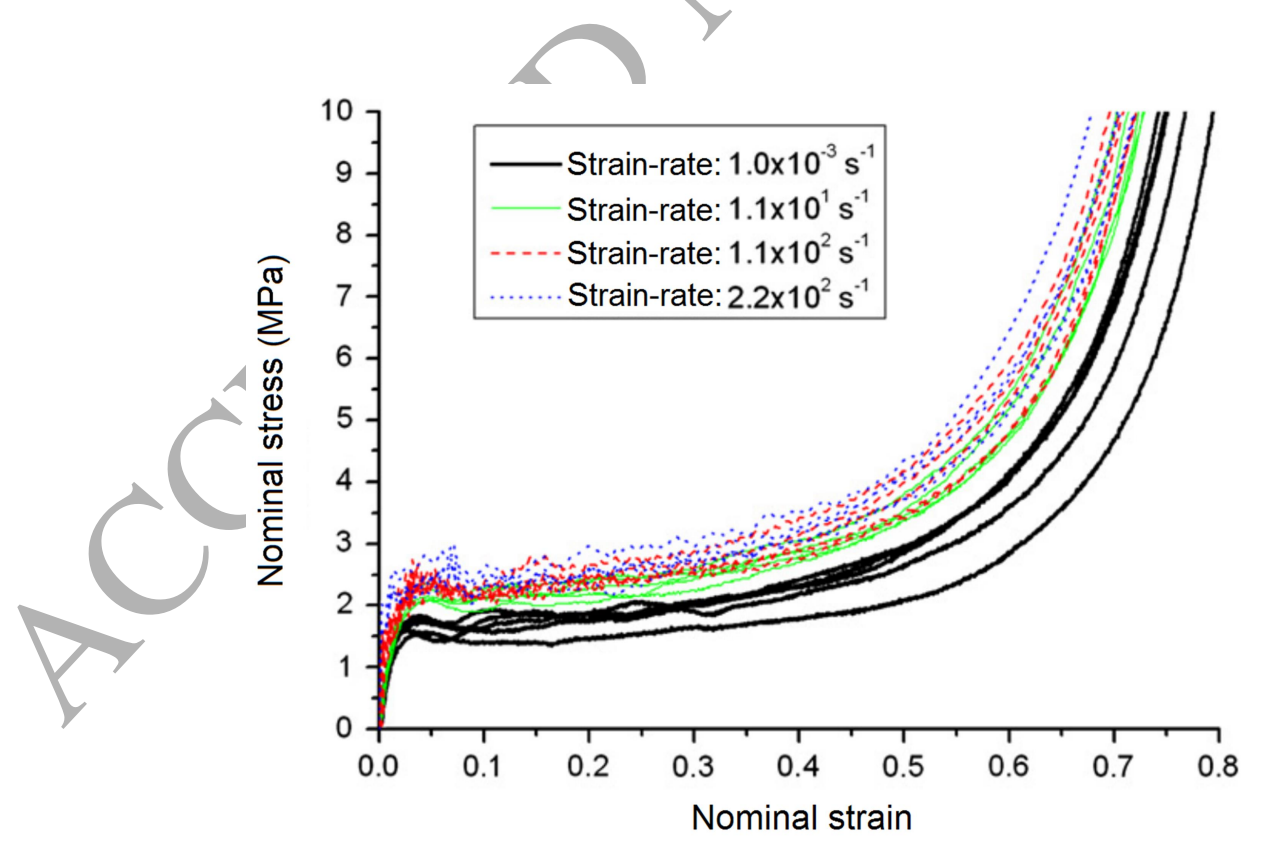

Fig. 6. Strain-rate effect on the compressive stress-strain relation of closed-cell aluminium Alporas foam [107]. 


\subsubsection{Strain-rate effect on plastic properties}

Extensive experimental tests have been conducted to examine the strain-rate effect on the plastic properties of cellular materials in the past two decades. However, it still lacks a general conclusion on the strain-rate sensitivity of cellular materials, and it is unclear whether some conflicting observations (discussed later) are caused by the limitation of experimental technique (see Section 2.2.1) or the inherent difference of dynamic compressive behaviour between different types of cellular materials.

Fig. 7 shows the dynamic compressive strength normalised by the quasi-static value for different types of cellular materials. The $20 \%$ scatter band, as suggested by Deshpande and Fleck [64], is included to consider the uncertainty in experiments. The strain-rate hardening is evident for the Balsa wood (loaded along grain) and the steel foam, but it is complicated for PVC and aluminium foams. The established mechanisms explain that the wood can be strengthened by micro-inertia (see Section 3.1.2), and the strain-rate sensitivity of steel foam is mainly due to the strong rate dependence of its base material, i.e. steel (See Section 3.1.1). However, these mechanisms cannot give convincible explanation about the dynamic compressive strength of PVC foams and aluminium foams. It appears that the PVC foams and the open-cell aluminium foams with higher densities are sensitive to strain-rate, but those with low densities are strain-rate insensitive. Despite the strain-rate insensitivity of some low-density polymer foams, most of polymer foams have been experimentally observed to be strain-rate sensitive $[29,61,94,121-125]$. It is also interesting to note that the strain-rate effect appears more pronounced at strain-rates above $1000 \mathrm{~s}^{-1}$, as shown in Fig. 7 and in Ref. [124]. However, cellular materials are generally susceptible to shock at moderate impact speeds (e.g. 40-60 m/s $[13,69,126])$, which can be comparable to the nominal strain-rates in the order of $10^{3} \mathrm{~s}^{-1}$, depending on the sample length and shock properties. Further discussion about this issue can be found in Section 2.3.

The closed-cell aluminium Alporas foam is evidently sensitive to strain-rate, see Fig. 6 and Fig. 7. By contrast, for another closed-cell aluminium foam (Cymat), Montanini [115] observed that it has a discernible rate-dependence of peak stress, but its plateau stress is insensitive to strain-rate. Therefore, it is necessary to further examine the strain-rate sensitivity in terms of plateau stress which represents the average of the stresses in the plateau stage. 


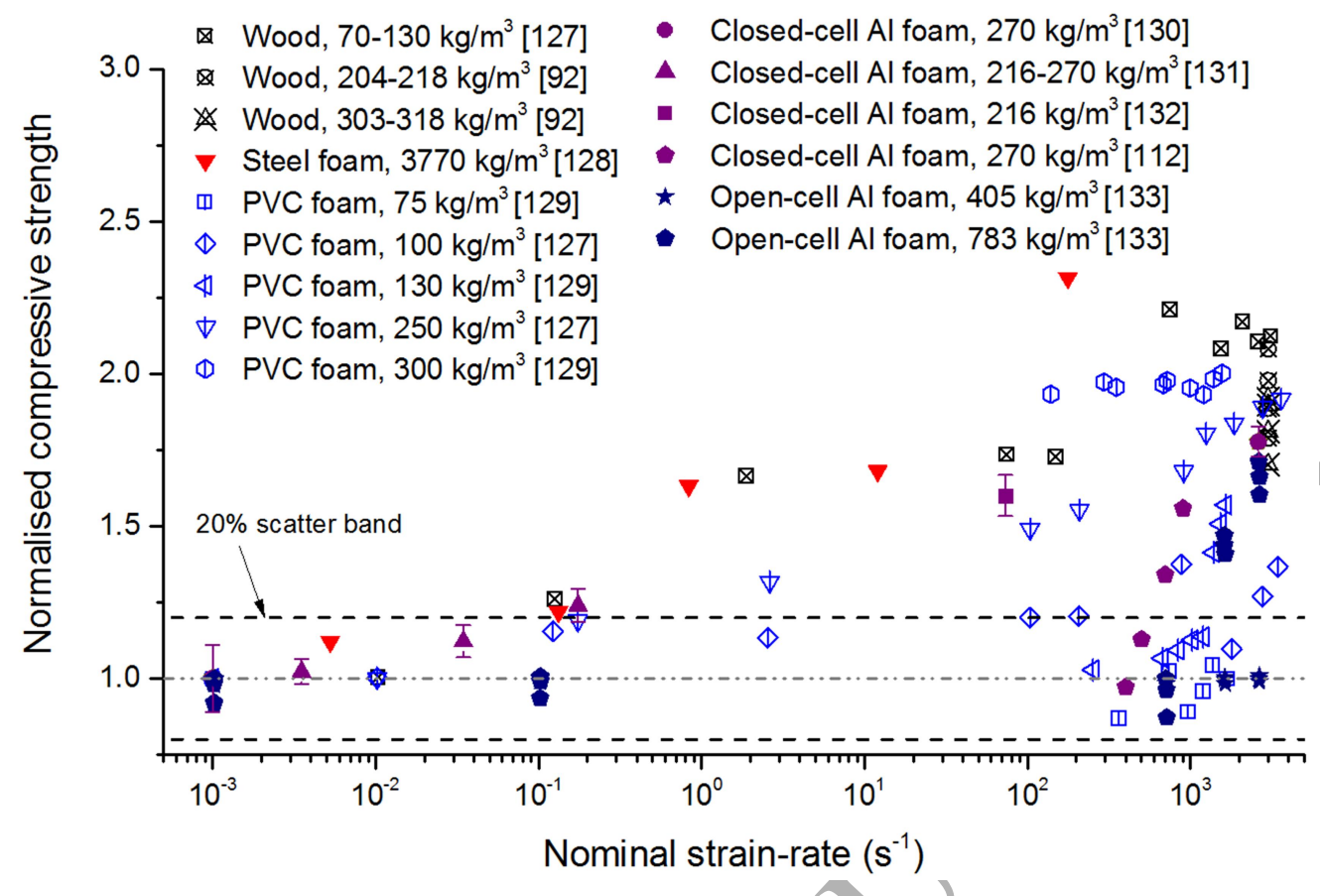

Fig. 7. Dynamic compressive strength normalised by the quasi-static compressive strength obtained at a nominal strain-rate of $0.001 \mathrm{~s}^{-1}[92,112,127-133]$. Note that the compressive strength is defined as collapse initiation stress or first peak stress, except the data of Ref. [127] where the stress at a nominal strain of 0.15 is used. Wood (Balsa) is loaded along grain.

Fig. 8 shows the plateau stress normalised by the relative density and the edge thickness-to-length ratio for closed-cell aluminium foams and aluminium honeycombs, respectively. It can be clearly seen that the plateau stress generally increases with strain-rate for Alporas foam, but it is insensitive to strain-rate for Alulight foam. Similar rate dependence has been observed for SCHUNK foam [115], IFAM foam [65] (Peroni et al. [134] observed an insignificant rate dependence of IFAM foam) and some non-commercial foams [135], while similar rate independence has been observed for Hydro foam [111], Cymat foam [115] and some non-commercial foams [136]. It implies that the cell structure, which may vary from each other among different types of foams manufactured by different processes, have a significant effect on the strain-rate sensitivity. This issue will be further discussed in Section 3. For aluminium honeycombs subjected to out-of-plane compression, the strain-rate hardening of plateau stress is evident, which has been consistently observed by different researchers [47, $65,137-139]$. Similar to foams, the cell structure of honeycombs affects the magnitude of this rate dependence (see mechanisms in Section 3). By contrast, the in-plane compressive strengths of aluminium honeycombs were observed to be insensitive to strain-rate [139]. 
(a)
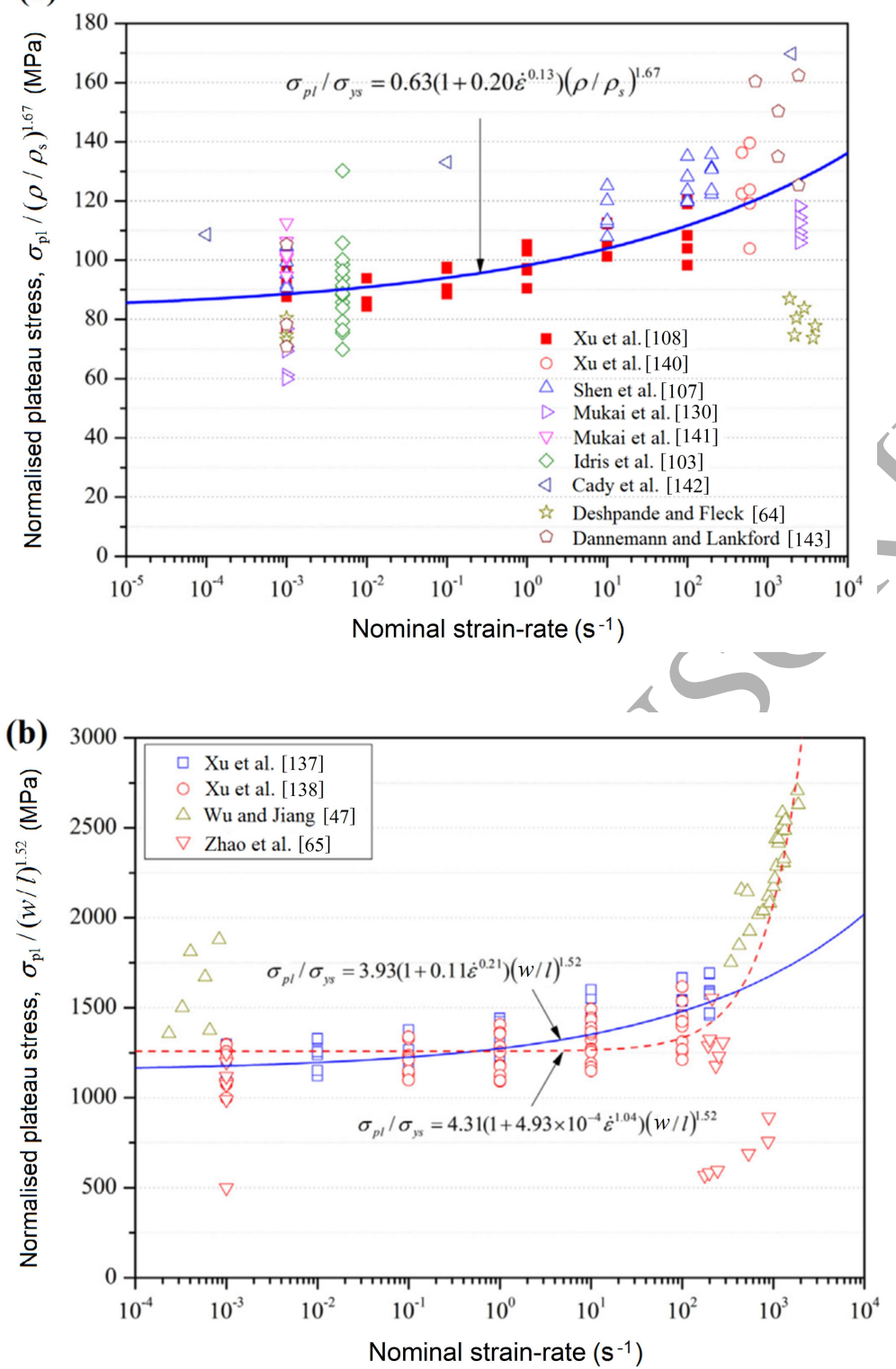

Fig. 8. Normalised plateau stress versus nominal strain-rate [108]: (a) closed-cell aluminium foams (note that the data reported by Deshpande and Fleck [64] are for Alulight foam, while all other data are for Alporas foam) [64, 103, 107, 130, 140-143]; (b) aluminium honeycombs (out-of-plane compression) [47, 65, 137, 138].

Densification initiation strain is another important parameter for the characterisation of plastic properties. However, some conflicting experimental observations have been reported for the strain-rate sensitivity of the densification initiation strain. Han et al. [144], Shen et al. [107], and Vural and Ravichandran [92] observed that the densification initiation strain decreases with increasing strain-rate for open-cell aluminium foam, closed-cell aluminium 
foam and balsa wood, respectively. Both Shen et al. [107] and Vural and Ravichandran [92] attributed this decrease in densification initiation strain to the less packing efficiency under dynamic compressive deformation. By contrast, Barnes et al. [69] and Tan et al. [145] reported that the densification initiation strain of open-cell aluminium foams increases with increasing strain-rate, which are consistent with the modelling results of Zou et al. [20]. However, Wang et al. [104] and $\mathrm{Xu}$ et al. [108] found that the densification initiation strain is almost independent of strain-rate according to their experimental results for closed-cell aluminium foams. The above inconsistency is probably caused by the inherent difference of the cell structures of the cellular materials tested or the different definitions of the densification initiation strain adopted. Based on energy absorption efficiency method, see Eq. (4), Sun and Li [34] found that the dynamic structural influence factor tends to increase the densification initiation strain of a $2 \mathrm{D}$ cellular solid, while the internal gas effect can lead to a decrease in it. The competition between these two mechanisms is dependent on both strain-rate and cell morphology.

The energy absorption capacity can be evaluated by calculating the dissipated energy per unit volume up to the densification initiation strain. However, when shock occurs, the energy absorption is complicated due to the strong discontinuity within the sample and the dependence of local stress-strain states on the loading speed, which will be discussed in next section. For many cellular materials compressed in the transitional dynamic regime, more energy can be dissipated when strain-rate increases [104, 107, 112, 131, 146], which are usually associated with the increases in plateau stress and/or densification initiation strain with the increase of strain-rate.

\subsection{Shock regime}

\subsubsection{Shock state and constitutive relation}

When the loading speed is sufficiently high, compaction shock occurs in most of cellular materials, and strong macro-inertia effect begins to play a dominant role in the dynamic compressive response. When shock occurs, the nominal stress-strain relation is unable to describe the constitutive behaviour of cellular materials and the nominal strain-rate should not be used to characterise dynamic behaviour, since the axial inertia leads to a significant difference between the stresses and strains across the shock front. This has been demonstrated in experiments and cell-based simulations, i.e. for a tested sample there is significant difference between the stresses measured at the loading and supporting ends, as well as the deformations observed in the crushed and uncrushed zones separated by the shock front. 
Therefore, it is impossible to establish stress equilibrium and strain homogeneity throughout the sample and measure the constitutive stress-strain relation. In order to obtain the stress and strain states under shock, the physical quantities of cellular materials must be determined locally [54, 69, 147-149].

The compaction shock discussed here pertains to a sudden loading (e.g. impact or blast loading) on a cellular material. Both constant speed loading and attenuating impact/blast are considered in this review, with emphasis on stress-strain state and constitutive relation in this section and dynamic response process in Section 4.2.1. The compaction shock is associated with a loading speed higher than a critical impact velocity (or equivalently, a critical blast pressure), which will be discussed in more details in Section 2.3.2. Other types of Shock [71, $109,150]$ represented by discontinuity but having stress/strain at the similar level of plateau stress/strain could also occur in cellular materials, but their existence requires more experimental and numerical evidence and thus they are not diseussed here. Although the continuum-based shock theory can be applied to most of cellular materials with a degree of success (see Section 4.2.1 for typical analyses), it should be noted that the shock in cellular materials is distinct from the shock in fully dense solids. Shock in cellular materials arises from mesoscopic structural mechanisms (e.g. flexural deformation, progressive cell collapse and compaction, see Section 3.2.1), and therefore, it is termed as "structural shock" [54, 55]. It is also noteworthy that the compaction shock in a cellular material can be initiated at a relatively low loading speed (e.g. 40-60 m/s $\lceil 13,69,126]$ ), and the shock speed in the cellular material is very close to the material particle velocity behind the shock front [25].

Reid and his collaborators pioneered in the experimental and theoretical studies in "structural shock" for energy absorbing structures and materials. "Structural shock" was initially used for $1 \mathrm{D}$ ring systems $[151,152]$, which was then extended to cellular materials like wood [153, 154] and foams [54, 55, 145]. Experimental setups used to generate compaction shock in cellular materials are shown in Table 1. In 1997, Reid and Peng [153] employed direct impact tests to investigate the dynamic compressive properties of five species of wood with impact velocity up to $300 \mathrm{~m} / \mathrm{s}$ and found significant strength enhancement measured at the impact end, which was attributed to shock enhancement. Subsequently, Tan et al. [54, 101] studied the dynamic compressive strength of closed-cell aluminium Hydro/Cymat foams using direct impact experimental setups with impact velocity ranging from 10 to $210 \mathrm{~m} / \mathrm{s}$, and they observed an approximately quadratic increase in dynamic plateau stress with impact velocity. They also showed that a critical impact velocity exists for a steady shock. More recently, Tan et al. [145] investigated the impact response of open-cell 
aluminium Duocel foam by the same experimental procedure and observed similar stress increase. In parallel, Lopatnikov et al. [148] and Radford et al. [147] employed a modified Taylor impact test, so-called Taylor cylinder-Hopkinson bar test, to investigate the dynamic compressive properties of closed-cell aluminium foams, and they also observed the shock enhancement of compressive strength.

Table 1 Impact experimental setups to generate compaction shock in cellular materials

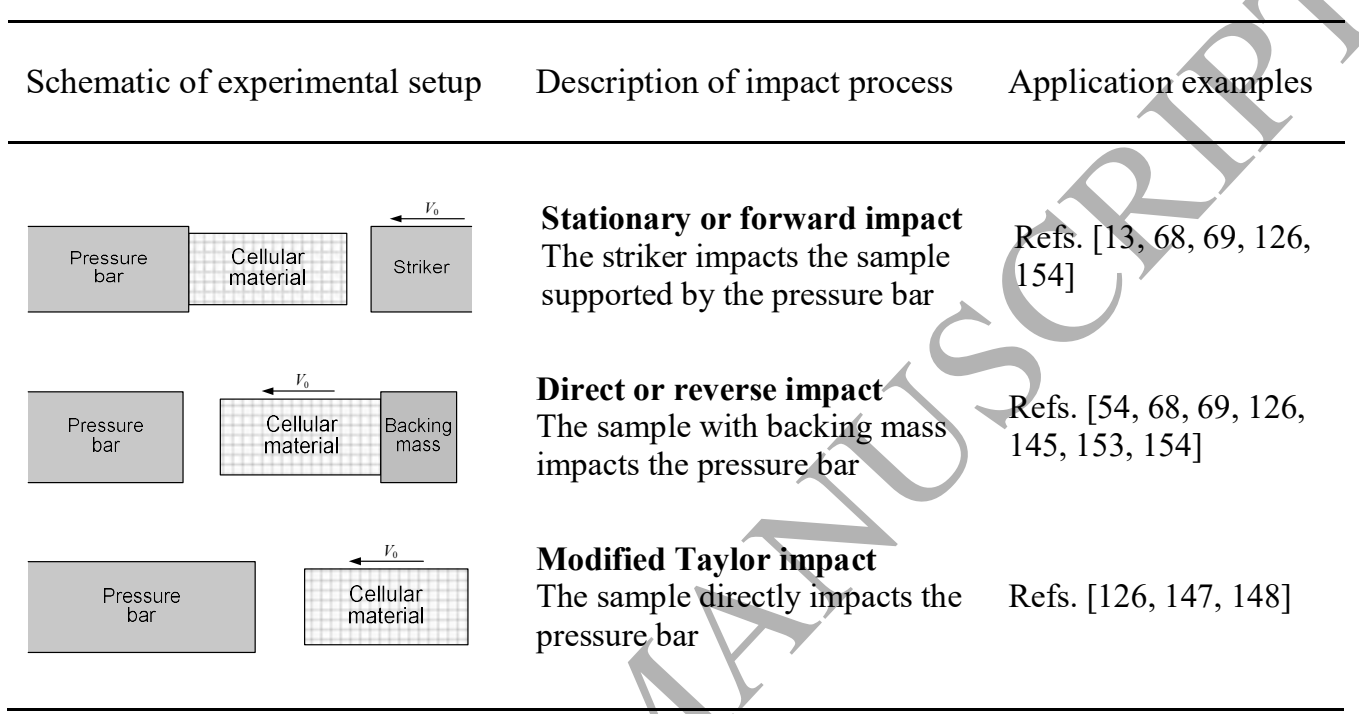

Note: The loading speed is inevitably decreasing when the input energy is absorbed during the impact test and thus the experiment represents a load attenuation process. However, when the input energy is sufficient (e.g. high initial velocity and/or large mass), the impact velocity is approximately equal to the initial velocity in the majority (or effective measurement) of the compression process. Strictly constant speed loading can be realised in simulations (see Figs. 31 and 32 in Section 4.1.2).

The experimental tests using direct impact and modified Taylor impact can only determine the dynamic crushing stress at the impact end. Due to this restriction, the aforementioned shock enhancement was evaluated by comparing the dynamic crushing stress with the quasi-static stress. It is not accurate if other mechanisms (see Section 3) are also responsible for the dynamic stress enhancement. To exclude the effect of the factors other than shock wave, if any exists, some researchers used the stationary or forward impact test to measure the stress ahead of shock front, and then compared this stress with the stress behind shock front obtained using direct or reverse impact test. For instance, Lee et al. [68] investigated the shock enhancement of open-cell aluminium Duocel foams and observed that the measured crushing stress at the impact end was significantly larger than that at the support end. Elnasri et al. [13] also employed this experimental strategy to investigate the dynamic 
crushing of honeycombs, foams and hollow sphere agglomerates, and they obtained similar results. Such a combination of direct and stationary impact tests is useful, as it enables the evaluation of the stresses at both the impact and support ends of the sample when shock occurs.

The "shock enhancement" observed in the aforementioned experimental studies can be interpreted as a jump of compressive stress across the shock front, which will be further discussed in following paragraphs and Section 4.2.1.

Although the shock effect on crushing stress has been extensively investigated in a quantitative manner, the shock deformation has not been quantified with satisfactory accuracy, due to the lack of experimental technique to accurately measure the local deformation or strain, which hinders the establishment of the stress-strain states under compaction shock. The early experimental observations of shock effect on cell deformation were mainly from cross-sectional images [54, 147, 148], which showed a sharp planar shock front separating crushed and uncrushed cells in cellular materials when subjected to high speed impact. This shock front represents a macroscopic discontinuity as defined in classical shock theory [150, 155] (see more discussion in Sections 3.2.1 and 4.1.2).

Digital image correlation (DIC) $[13,68,109]$ was later used to quantify and visualise the shock deformation. Fig. 9 shows the local strain distributions along the axis of shock compression, which were measured by DIC in impact tests [13]. This quantitative examination of the shock deformation demonstrates the jump of local strain across the shock front even at rather moderate loading speeds $(40-60 \mathrm{~m} / \mathrm{s})$. Such a big strain jump also justifies the treatment of shock front as a discontinuity at the macro-scale. 

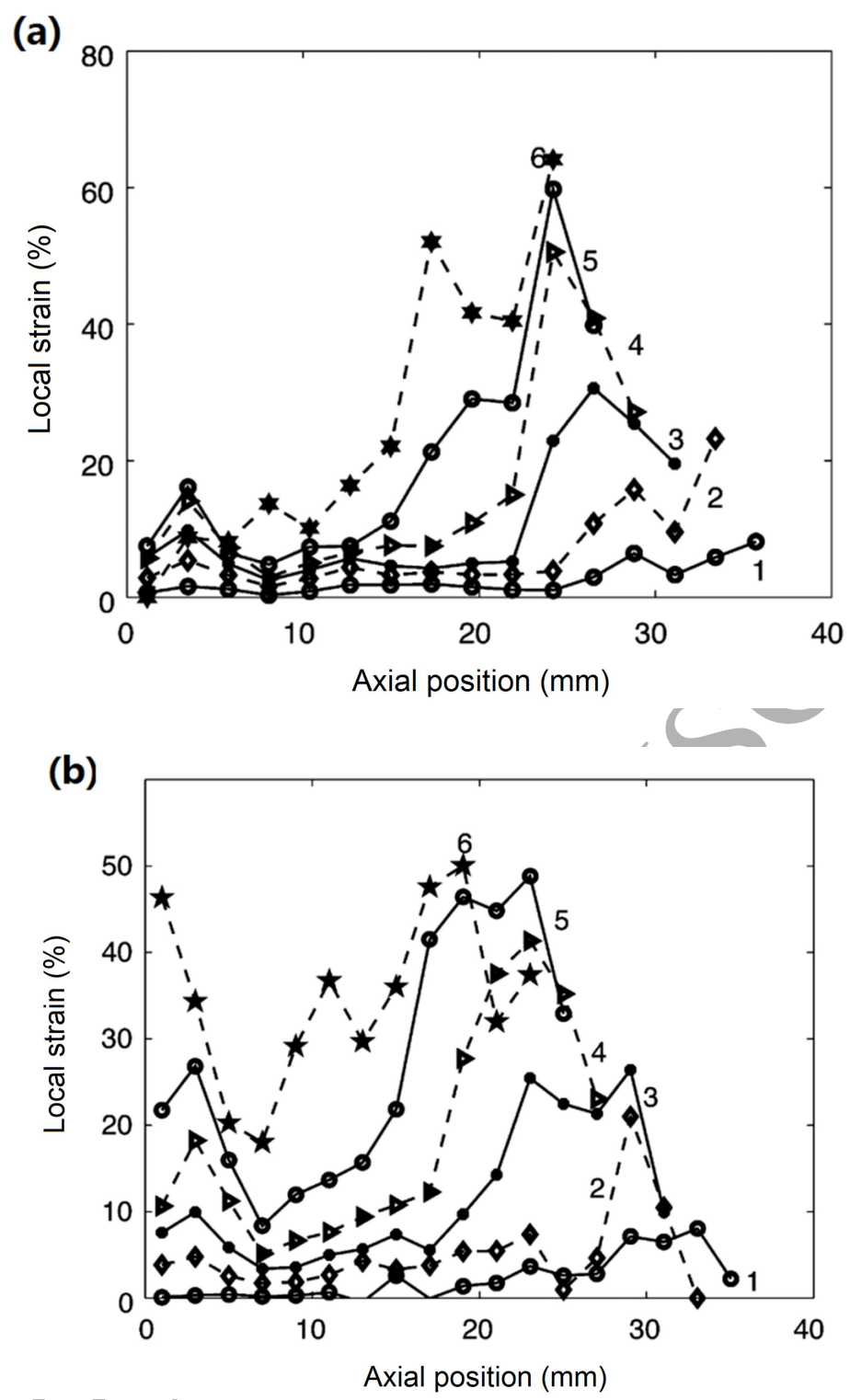

Fig. 9. Distribution of the axial local strain (DIC measurement) in a foam sample impacted at: (a) $47 \mathrm{~m} / \mathrm{s}$; (b) $56 \mathrm{~m} / \mathrm{s}$ (solid line: even numbers of images; dashed line: odd numbers of images) [13].

The experimental DIC technique, however, often suffers difficulty and errors for local strain measurement when the deformation is large $[18,31]$, which is certainly troublesome for the shock compression that always causes severe cell crushing (see Fig. 10 and Fig. 11). Nevertheless, it has been demonstrated that the shock front and its propagation speed can be determined by combing high speed imaging and DIC [13, 69]. Fig. 10 shows the formation and propagation of the shock front in an open-cell aluminium foam [69]. This enables the measurement of local engineering strain behind the shock front (shock strain for brevity) [25, 
69], as long as the position of the planar shock front is known. When loading speed is constant or approximately unchanged, the shock strain can be obtained as (see Fig. 11 for illustration)

$$
\varepsilon_{\mathrm{b}}=\frac{H_{\mathrm{b}}-h_{\mathrm{b}}}{H_{\mathrm{b}}}=\frac{H-h_{\mathrm{a}} /\left(1-\varepsilon_{\mathrm{a}}\right)-h_{\mathrm{b}}}{H-h_{\mathrm{a}} /\left(1-\varepsilon_{\mathrm{a}}\right)} \approx \frac{H-h_{\mathrm{a}}-h_{\mathrm{b}}}{H-h_{\mathrm{a}}}=\frac{H-h}{H-h_{\mathrm{a}}}
$$

where $H_{\mathrm{a}}$ and $H_{\mathrm{b}}$ are the initial heights (or lengths) of the uncrushed and crushed zones, which are ahead of and behind the shock front, respectively, and correspondingly $h_{\mathrm{a}}$ and $h_{\mathrm{b}}$ are defined in the current configuration; $H$ and $h$ are the initial and current sample heights (or lengths), respectively; $\varepsilon_{\mathrm{a}}$ is the local engineering strain in the uncrushed zone. An approximation, i.e. $\varepsilon_{\mathrm{a}} \approx 0$, can be made, see Eq. (8), as the deformation in the uncrushed zone is much smaller than the shock deformation. If the impact velocity varies significantly during shock propagation, a modified form of Eq. (8) may be used, i.e. $\varepsilon_{\mathrm{b}_{-} k}=\left(\Delta H_{\mathrm{b}_{-} k}-\Delta h_{\mathrm{b}_{-} k}\right) / \Delta H_{\mathrm{b}_{-} k} \approx \Delta h_{k} / \Delta h_{\mathrm{a}_{-} k}$ where $\varepsilon_{\mathrm{b}_{-} k}$ is the shock strain at moment $k$, $\Delta H_{\mathrm{b} \_k}=H_{\mathrm{b} \_k}-H_{\mathrm{b}_{\_} k-1}, \Delta h_{\mathrm{b} \_k}=h_{\mathrm{b} \_k}-h_{\mathrm{b} \_k-1}, \Delta h_{k}=h_{k}-h_{k-1}$ and $\Delta h_{\mathrm{a} \_k}=h_{\mathrm{a} \_k}-h_{\mathrm{a} \_k-1}$.

It should be noted that the local engineering strain calculation using Eq. (8) is actually based on the averaging of cell deformation in the crushed zone which encompasses an increasing number of cells during shock propagation. This is different from some other strain measures reported in the literature, e.g. DIC local strain [13], Lagrangian method strain [109, 156], cross-sectional averaging strain [20] and nodal neighbourhood strain [22, 23], which all depend on the size of the seleeted sub-region (typically at a scale of average cell size) and may not be able to reflect macroscopic behaviour of collective cells if cell heterogeneity is significant. In this sense, the strain measure defined by Eq. (8) is convenient and advantageous owing to its consistence with the nominal strain measure (i.e. the engineering strain defined over the sample gauge length) widely used in quasi-static and transitional dynamic compressions (see Fig. 4 and Fig. 6). 
(a)

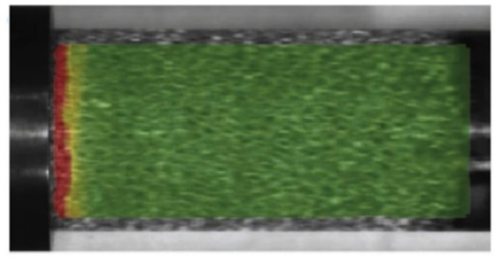

(1)
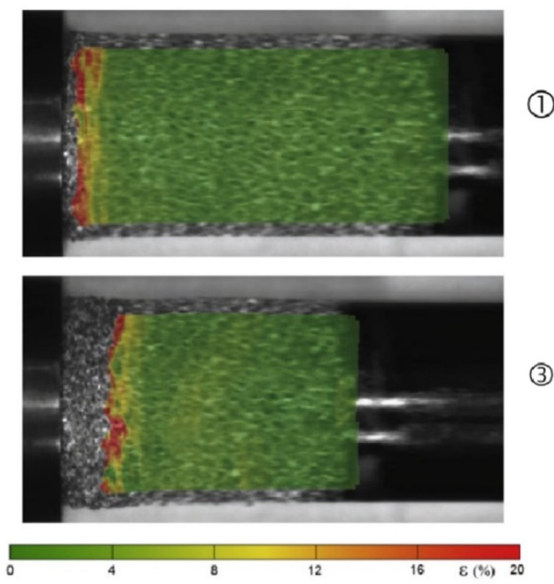

(b)

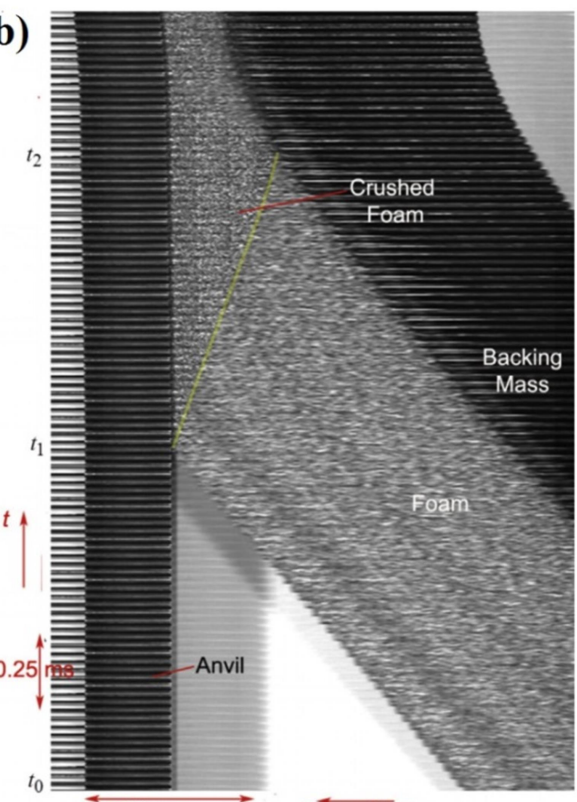

Spec. Motion

Fig. 10. Formation and propagation of shock front in open-cell aluminium foam (adapted from Ref. [69]): (a) DIC strain contours superposed on the high speed digital images; (b) position vs. time diagram of a series of slices (extracted from the corresponding 2D images) of the foam from a direct impact experiment with an initial impact velocity of $90 \mathrm{~m} / \mathrm{s}$ (note: the yellow line indicates the trajectory of shock front).

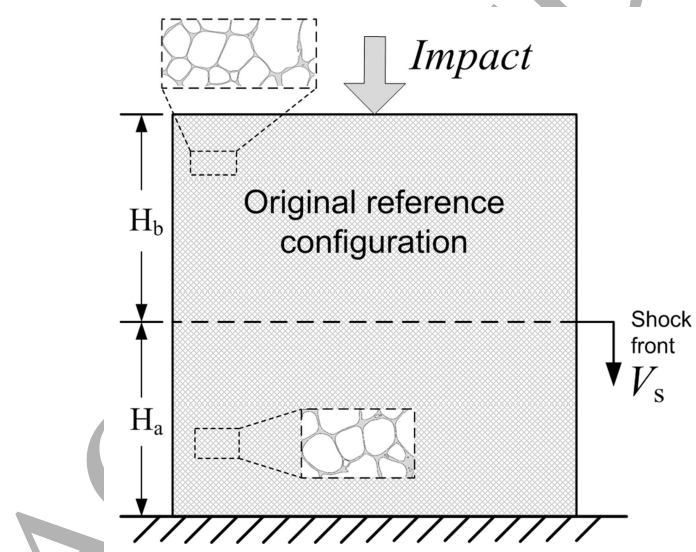

(a)

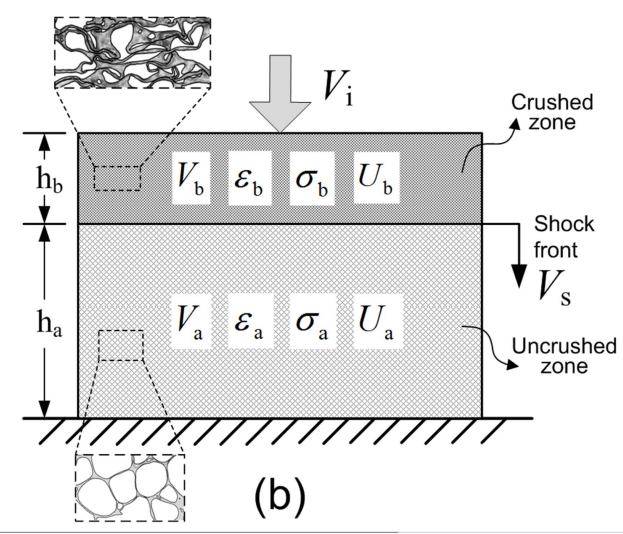

Fig. 11. Schematic of the shock front propagation [25]: (a) the position of the shock front in the original reference configuration (Lagrangian configuration); (b) the position of the shock front, and the shocked material states, in the current configuration (Eulerian configuration). 
Applying the technique described above, Barnes et al. (2014) [69] experimentally measured the local physical quantities and established the Hugoniot relations (i.e. loci of all shock state variables), as shown in Fig. 12. It reveals that the shock speed, strain, stress and strain energy density all increase with impact velocity. In particular, the shock speed increases with impact velocity approximately linearly and the linear relation is expressed as

$$
V_{\mathrm{s}}=V_{\mathrm{r}}+S V_{\mathrm{b}}
$$

where $V_{\mathrm{b}}$ is impact velocity (or material particle velocity behind the shock front), $V_{\mathrm{r}}$ is a reference speed (i.e. $V_{\mathrm{s}}$ intercept), and $S$ is a material parameter. This linear relation implies an increasing plastic wave speed with strain, which satisfies the general requirement of shock formation in solids, as stated in the shock theory $[25,150]$. For many metallic foams, $S$ is approximately equal to one [25, 69, 157-159], while for some polymer foams $S$ is larger than one, i.e. 1.00-1.35 $[160,161]$. When $S$ is larger than one, an upper limit of shock strain, i.e. $1 / S$, can be estimated $[25,160,161]$. In general, $S$ is almost a constant $(\approx 1)$ for most of cellular materials, while $V_{\mathrm{r}}$ can vary in a wide range for different types of cellular materials. Numerical simulations of 3D Voronoi foams indicate that $V_{\mathrm{r}}$ is dependent on relative density, entrapped gas and strain/strain-rate hardening of base material [157]. It is also interesting to note that, according to some experimental and numerical results, $V_{\mathrm{r}}$ seems close to the critical impact velocity for shock initiation [25, 69, 158, 159] (see Section 2.3.2 for more discussion of shock initiation).

Similar linear relation has been also observed in other engineering materials $[155,160$, 162]. For cellular materials the constant $V_{\mathrm{r}}$ in Eq. (9) is much smaller than the sound speed $C_{0}$, while for many traditional engineering materials $V_{\mathrm{r}} \approx C_{0} \quad[25,69,162]$. As the coefficient $S$ is close to one for most of cellular materials $[25,69,157]$, the shock speed is only slightly larger than the impact velocity, and the difference is approximately equal to $V_{\mathrm{r}}$. 
(a)

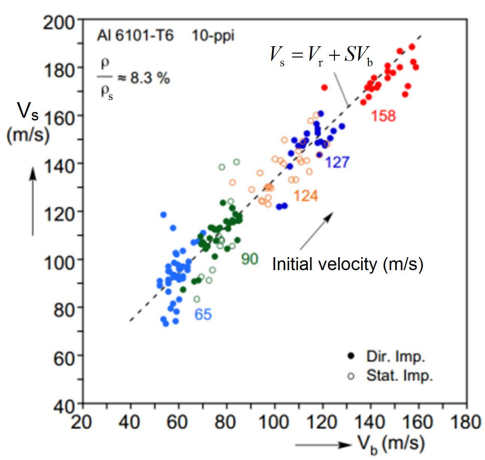

(c)

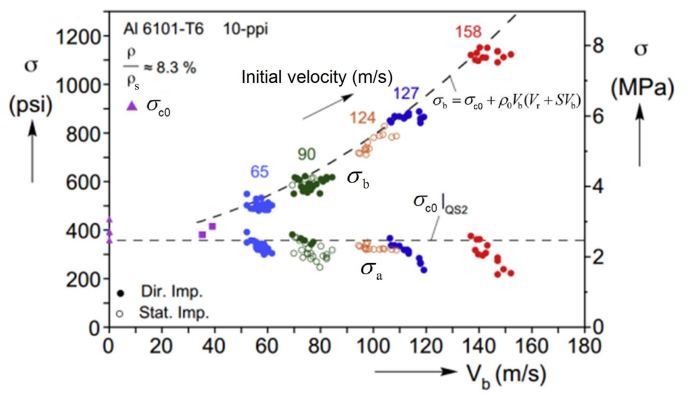

(b)

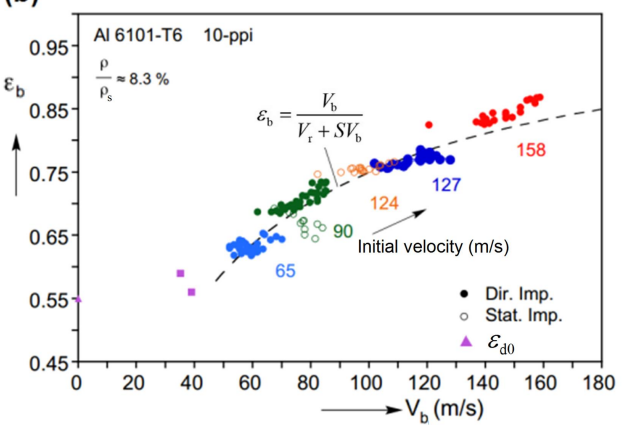

(d)

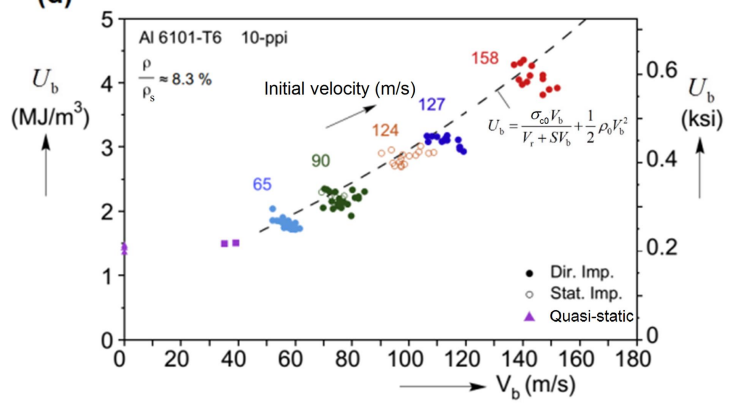

Fig. 12. Hugoniot relations for open-cell aluminium Duocel foam [69]: (a) shock speed vs. impact velocity; (b) shock strain vs. impact velocity; (c) shock stress vs. impact velocity; (d) shock strain energy density vs. impact velocity. The equations corresponding to the dashed lines are derived from the linear Hugoniot relation, see Ref. [69].

Barnes et al. [69] further correlated the stress and strain behind the shock front, and they found that the stress-strain Hugoniot relation of the shocked foam is distinct from both the quasi-static and transitional dynamic stress-strain relations, as shown in Fig. 13a. Therefore, they pointed out that "a constitutive model based on the quasi-static crushing response is not appropriate in determining this Hugoniot or the variables not determined directly from impact experiments". A significant difference in the stress-strain relation between shock compression and quasi-static compression was observed also in an experiment of polyurethane foam [161]. These findings are important for evaluating the errors of prevalent shock analysis models using the quasi-static stress-strain relation as a constitutive model (see more discussion in Section 4.2.1).

In parallel, numerical simulations also provided valuable results to clarify this issue. A systematic research has been conducted at USTC, mainly based on numerical modelling of Voronoi foams. In 2013, Liao et al. [22] employed a nodal neighbourhood strain method to calculate the local strain and established the shock stress-strain states for a 2D Voronoi foam. In 2014, Zheng et al. [149] extended the local strain calculation to a 3D Voronoi foam and showed that a dynamic stress-strain equation (dynamic, rigid, non-linear hardening plastic: 
D-R-NLHP) can be used to fit the locus of shock stress-strain states for a cellular material, which is distinct from the quasi-static stress-strain relation, as shown in Fig. 13b. Each shock stress-strain state corresponds to an impact velocity, and the locus of all shock stress-strain states at different impact velocities can be described by a unique dynamic shock stress-strain relation. By contrast, in the transitional dynamic regime, different strain-rates (or loading speeds) actually leads to different stress-strain relations (Fig. 6), as discussed by Zheng et al. [149]. Zheng et al. [149] further proposed the below equation to describe the dynamic shock stress-strain relation

$$
\sigma=\sigma_{0}^{\mathrm{d}}+D \frac{\varepsilon}{(1-\varepsilon)^{2}}
$$

where $\sigma_{0}^{\mathrm{d}}$ and $D$ are material constants. The form of Eq. (10) is similar to that of the stress-strain Hugoniot relation derived from linear $V_{\mathrm{s}}-V_{\mathrm{b}}$ relation (see deriyation in Refs. [25, 69]), i.e.

$$
\sigma=\sigma_{0}^{\mathrm{sh}}+\rho_{0} \varepsilon\left(\frac{V_{\mathrm{r}}}{1-S \varepsilon}\right)^{2}
$$

where $\sigma_{0}^{\text {sh }}$ is a stress constant (approximated as quasi-static collapse initiation stress $\sigma_{\mathrm{c} 0}$ in Refs. $[25,69])$ and $\rho_{0}$ is the initial density before loading. It can be seen that Eq. (10) is actually a special form of Eq. (11) when $S=1$. It was noticed in Ref. [25] that Zheng et al. [149] chose a function consistent with the linear $V_{s}-V_{\mathrm{b}}$ relation to establish the description of shock stress-strain states in a 3D Voronoi foam, but in general the choice of empirical nonlinear functions may not be unique (see Section 4.2.1) to achieve good fitting of numerical or experimental data. Therefore, it is recommended that the unique linear $V_{\mathrm{s}}-V_{\mathrm{b}}$ relation is preferably used for the description of shock states in cellular materials [25].

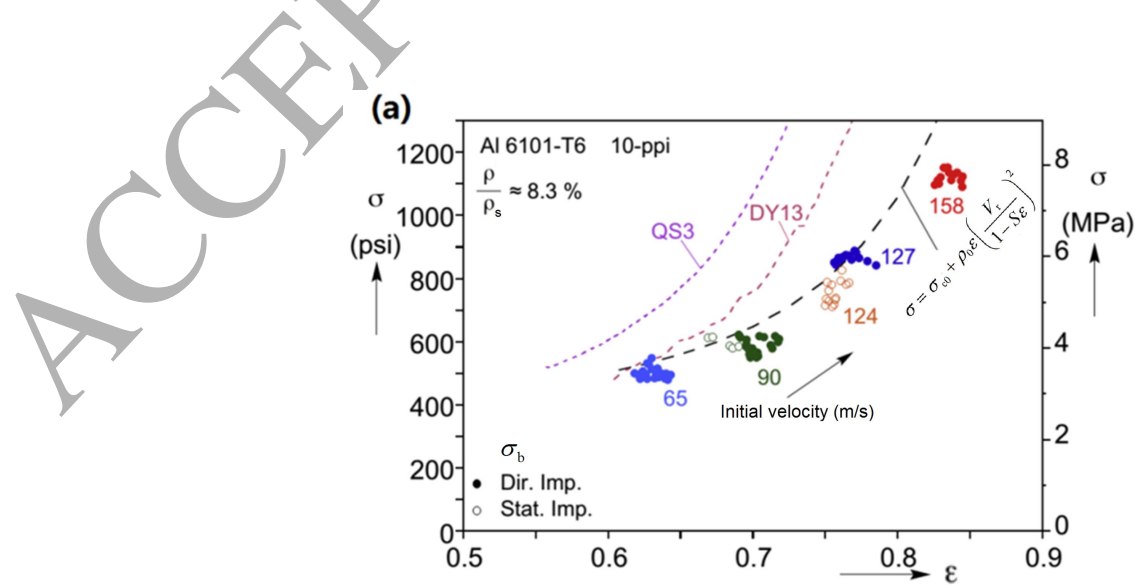




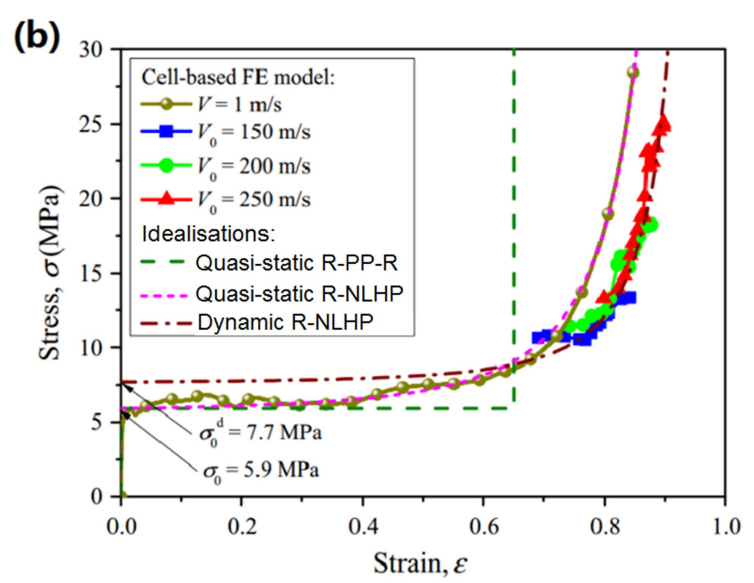

Fig. 13. Shock stress-strain states for: (a) open-cell aluminium Duocel foam [69] (QS3: quasi-static test; DY13: dynamic test at an impact velocity of $35.1 \mathrm{~m} / \mathrm{s}$ ); (b) $3 \mathrm{D}$ closed-cell Voronoi foam [149] (R-PP-R: rigid, perfectly plastic, rigid; R-NLHP) rigid, nonlinear hardening plastic).

The above discussion considers mainly the material's stress-strain states in the crushed zone behind the shock front. Less accuracy of experimental measurement of the state variables can be achieved for the uncrushed zone ahead of the shock front, due to the relatively small strain and stress therein. In experimental tests, it is unlikely to directly measure the stresses within a sample, so the stresses measured at two ends of the tested sample are usually used to characterise the shock stresses. Barnes et al. [69] neglected the deformation in the uncrushed zone and found that the stress ahead of the shock front is bounded by the quasi-static/collapse initiation stress (Fig. 12c). This leads to the simplification of a constant stress and zero strain state ahead of the shock front, which is also supported by some numerical simulations [22, 26, 59, 158, 163]. However, Zheng et al. [149] found that the stress ahead of the shock front, so-called dynamic initiation stress, is larger than the quasi-static collapse initiation stress for a 3D Voronoi foam. They further demonstrated that the "dynamic initiation stress", even when the base material is strain-rate insensitive, is dependent on local strain-rate [156], rather than a constant as adopted early by the same research group [149, 157]. However, it should be noted that in Zheng and his co-workers' numerical studies $[149,156,157]$ the local stress "immediately ahead of shock front" was used, whereas, in the experiment by Barnes et al. [69] and the simulations by other researchers $[22,26,59,158,163]$, the stress at the support end was used. It has been numerically shown that the collapse stress determined at the support end of a foam sample is sensitive to the properties of the cells adjacent to the end, and thus the dynamic collapse stress can be either slightly smaller or larger than the quasi-static collapse stress, depending on the 
locations of measurement (Fig. 17) [26]. The reflection of precursor elastic wave at the support end may further complicate the stress measurement there. On the other hand, the location "immediately ahead of shock front" has not been defined strictly [25] and the measurement immediately ahead of the shock front is difficult due to the finite thickness of the shock front at the meso-scale. The aforementioned difference in stress measurement may partially explain the different conclusions drawn by different researchers.

Recalling Eq. (10) and Eq. (11), which have been shown to be capable of describing the stress-strain states of the material behind the shock front $[25,69,149,158]$, it is still a question whether same relation can be applied to the material ahead of the shock front. For uncrushed zone, Eq. (10) and Eq. (11) imply that precursor elastic wave exists, However, dynamic elastic wave dispersion was experimentally observed in polymer foams [164], and the actual elastic wave speed of a Voronoi foam was shown larger than that calculated using effective Young's modulus [70]. The elastic (and plastic) wave formed in the base material and propagating in the mesoscopic structural components (e.g. struts and walls) can be much more complex than the structural shock wave propagating at the macro-scale $[20,165,166]$. It is still unclear whether the bulk elastoplastic properties defined in classical continuum mechanics are sufficient to describe the propagation of different types of stress waves in cellular materials, while classical continuum mechanics has achieved a remarkable success to analyse the macroscopic shock wave (see Section 4.2.1). The transmission and reflection of the precursor wave arriving at the support end further complicates the problem. Indeed, the stress measured at the support end exhibits complex variation during the shock propagation in forward (or stationary) impact test $[13,68,126]$. If precursor stress waves produce a macroscopic plastic strain that is relatively large, Eq. (10) or Eq. (11) could be used to estimate the stress ahead of the shock front, given that the elastic deformation is negligible. Alternatively, material parameters, e.g. $\sigma_{0}^{\text {d }}$ in Eq. (10) and $\sigma_{0}^{\text {sh }}$ in Eq. (11), can be regarded dependent on local strain-rate when shock propagates, as suggested in Ref. [156], which, however, may be more difficult to determine in practice. The relatively small stress and strain in the uncrushed zone may not be worth too much extra effort into marginal improvement of the accuracy of measurement or prediction. To resolve the remaining issues in the fundamental aspect, more experimental and numerical studies of stress waves propagating at the meso-scale are needed.

For the material within the narrow shock front examined at the meso-scale, the stress-strain states follow a linear Rayleigh chord, as predicted by shock theory $[150,167]$ and observed in numerical simulations [156]. 


\subsubsection{Critical condition for shock initiation}

It is of both fundamental importance and practical interest to determine and predict the critical condition for the initiation of compaction shock. Experimental observations (see Section 2.3.1) and numerical simulations (see Section 4.1.2) have clearly demonstrated the features of the compaction shock in cellular materials $[20,22,54,148,153]$. These features are consistent with those of shock observed in fully dense solids $[71,109,150]$, i.e. the existence of a macroscopic strong discontinuity or a mathematical singular interface for the first-order derivatives of displacement (e.g. velocity and strain), and the propagation of macroscopic planar wave front. Wang et al. [71, 109] proposed two critical velocities for plastic shock wave in cellular materials, i.e. the lower critical velocity is the impact velocity corresponding to yielding, while the upper critical velocity is the impact velocity corresponding to densification. It appears that this is an approach to obtain the critical impact velocity for shock initiation from lower side in the axis of loading rate (Fig. 3) (i.e. from nonshock to shock), wherein stress wave propagation is considered in both elastic (or pre-collapse) regime and plateau regime. However, this theoretical classification of critical condition does not agree with experimental and numerical results consistently (see Sections 2.3.1 and 3.2.1), partially because of the stress oscillation in the plateau regime (see Fig. 4 and Ref. [96, 168]). The significant stress oscillation in the plateau stage exists mainly due to cell collapse and thus it is sensitive to mesoscopic structural heterogeneity or defects. As strain softening or decreasing strain hardening can lead to wave divergence [150], steady shock in cellular materials requires that the loading intensity is sufficiently high, usually reaching the densification stage which is featured by cell compaction and smooth stress-strain curve with increasing slope. This requirement is normally unnecessary for fully dense solids which have relatively smooth stress-strain curve throughout the elastic-plastic regime and less mesoscopic structural heterogeneity than cellular materials (e.g. foams). The supposed plastic shock wave controlled by the lower critical impact velocity will be referred to as non-compaction shock and will be distinguished from the most widely observed compaction shock in cellular materials, as discussed later.

The difference between non-compaction shock and compaction shock has been actually involved in some theoretical and numerical studies, despite different terminology adopted. For instance, Zheng et al. [169] used the term "shock" specifically for localised and progressive densification, i.e. the local strain behind shock front reaches or exceeds the densification initiation strain. They proposed a "transitional-mode" for the plastic stress wave with smaller local strain behind the discontinuity wave front. Nevertheless, they employed the 
same continuum-based shock model to analyse both "shock-mode" and "transitional-mode". Recently, Li et al. [170] argued that the mesoscopic Lagrangian width of "shock" front should not exceed the average cell size based on a 3D Voronoi model; otherwise the deformation should be termed as "band front mode". This may shed some light into the mesoscopic difference between the "shock mode" and "transitional-mode" proposed by Zheng et al. [169]; but the method using the average cell size to distinguish two different wave modes did not consider the variation of cell sizes in a Voronoi model. On the other hand, the macroscopic shock is distinct from the mesoscopic wave propagation via cell walls/struts under shock loading. Kader et al. [165] demonstrated that the precursor elastic and plastic waves actually propagate in convoluted paths in foams subjected to shock and their propagation dépends on cell topology in foams.

For a general description, the shock, defined as the formation of a strong discontinuity wave front propagating from loading end, can be subcategorised into non-compaction shock and compaction shock in a cellular material. For the former, the cells behind the shock front are not densified, while for the latter the cells are densified to a certain extent. The latter is thus called compaction or densification wave by some researchers [e.g. 171, 172]. It should be noted that the concept of non-compaction shock may be related to the numerically observed "transitional-mode" [67, 169] and "band front mode" [170], and it produces a shock strain between collapse initiation strain and densification initiation strain [71, 109]. However, the moderate cell deformation is sensitive to the unstable collapse mechanism and the mesoscopic structural heterogeneity/defects [31, 173], which can introduce local stress softening (see the stress oscillation in Figs. 4, 6, 25, 26 and 27) and potentially lead to wave divergence [150], as mentioned earlier. To produce steady non-compaction shock, the cellular material should exhibit smooth stress-strain relation and continuous increasing (or linear) strain-hardening in the plateau stage. However, a relatively flat stress plateau (usually with stress oscillation) is most commonly observed for cellular materials. Since it is difficult to produce the non-compaction shock in common cellular materials, we refer shock to compaction shock in the subsequent part of this review, unless otherwise stated.

The critical condition for shock initiation has been predicted mainly based on two criteria: stress enhancement and strain increase [25, 59]. In other words, the jumps of stress and strain are selected as two indexes to evaluate the magnitude of the discontinuity across the presumably formed shock front. In general, a stress enhancement with respect to the quasit-static plateau stress by $0.1-2.0$ times is used $[3,54,55]$; and the critical strain is usually adopted to be the collapse initiation strain [71] or densification initiation strain $[25,106,169]$, 
as predefined using quasi-static stress-strain relations. In experiments and simulations, the shock initiation can be evaluated according to the deformation mode, i.e. the formation of shock front $[13,20,69,158]$. In Table 2 [25], the critical impact velocity $\left(V_{s-c}\right)$ for shock initiation is predicted using different analytical methods and compared between each other, which shows that the Hugoniot relation can be used to predict the critical impact velocity with better accuracy. The Hugoniot-based method proposed in Ref. [25] is essentially approaching the critical impact velocity from the upper side in the axis of loading rate, as shown in Fig. 3, i.e. from compaction shock to transitional dynamic compression or supposed non-compaction shock. This method relies on the high speed impact tests where compaction shock already occurs. Despite the prediction accuracy, more experimental and numerical test data are still needed to unify the different approaches to obtain the critical condition for the initiation of compaction shock.

It should be noted that current evaluation of shock in cellular materials is mainly based on macroscopic quantities (e.g. velocity, strain and stress). The critical condition for shock initiation is thus identified by the observation of the macroscopic discontinuity in tested samples, and the prediction of the critical impact velocity relies on the macroscopic constitutive relation (e.g. stress-strain relation or/Hugoniot relation). The governing mechanism of shock in cellular materials is generally attributed to the inertia of cell walls/struts and the compaction of cells, see discussion in Section 3.2.1. However, their mesoscopic mechanisms have not been completely established. 
Table 2 Predictions of the critical impact velocity, $V_{s-c}$, for shock initiation in cellular materials using different methods (unit: $\mathrm{m} / \mathrm{s}$ ) [25]

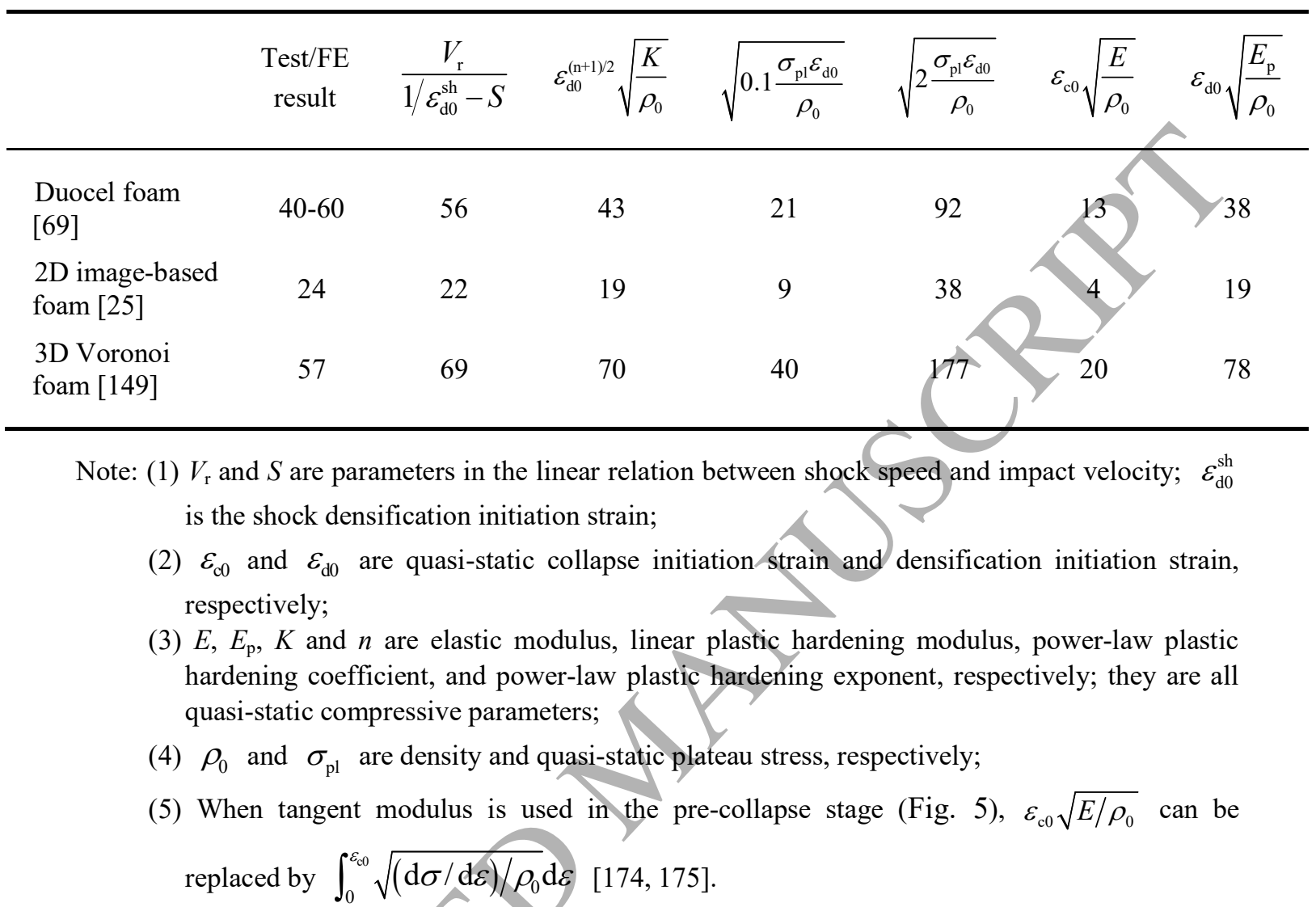

\section{Mechanisms}

\subsection{Micro-scale: base material}

\subsubsection{Microscopic strain-rate sensitivity}

Many dense solids (e.g. metals and polymers) can be used as raw materials or matrix materials for the fabrication of cellular materials. These raw materials can be strain-rate sensitive themselves due to microscopic mechanisms (e.g. thermal activation, photon drag and relativistic effect) $[162,176]$. One example is steel, which is generally strengthened by increasing strain-rates $[162,177]$, and typically, the yield stress of a mild steel is doubled when strain-rate increases from $10^{-4}$ to $40 \mathrm{~s}^{-1}$ [177]. Another example is polymer, which in general exhibits significant strain-rate hardening, e.g. PP has a peak stress increasing from 34 $\mathrm{MPa}$ at $10^{-2} \mathrm{~s}^{-1}$ to $67 \mathrm{MPa}$ at $20 \mathrm{~s}^{-1}$ [178]. However, the strain-rate sensitivities of aluminium and some other metals are more complex $[179,180]$. For instance, the strain-rate 
corresponding to a $100 \%$ increase of yield stress, compared to static case, can vary in the range between $6500 \mathrm{~s}^{-1}$ and $1288000 \mathrm{~s}^{-1}$ for aluminium alloy, depending on the exact material specification [177].

Base material is the solid constituent of a cellular material, and the mechanical properties of base material may be changed due to the manufacturing process, and consequently they may be different from those of the raw material. The dominant microscopic mechanisms for the strain-rate sensitivity of a cellular material are closely related to the microstructure of the base material. It has been demonstrated in experiments that the strain-rate hardening of aluminium alloy foams can be significantly enhanced by modifying the constituent elements and microstructures of the aluminium alloys [181]. On one hand, the discrete obstacles to dislocation motion in the raw material have significant implications in the strain-rate sensitivity of the base material. On the other hand, for a specific cellular/material, the base material can be substantially changed from the raw material during manufacturing. For instance, the closed-cell Alporas foam, which is produced by adding a thickening agent of 1.5 wt.\% $\mathrm{Ca}$ into molten aluminium and stirring the mixture with a blowing agent of $1.6 \mathrm{wt} . \%$ $\mathrm{TiH}_{2}$ at $680{ }^{\circ} \mathrm{C}$ [182], has pure aluminium as its raw material, but its base material is actually an aluminium alloy with additional elements and modified microstructure after fabrication. In Fig. 14, it is clearly seen that considerable precipitates, micropores and coarse particles exist in the cell walls. Such microscopic heterogeneity has been widely observed in aluminium foams, which can lead to distinctive mechanical properties of the base material [166, 183-185]. It has been also demonstrated that a heterogeneous material made of different rate-independent phases can exhibit strain-rate sensitivity due to the heterogeneity [186]. The complication of the microstructure after fabrication causes particular difficulty to evaluate the strain-rate sensitivity of the base material, as dynamic tests need to be directly performed on the cell walls/struts, which, however, are too small for conventional test methods (e.g. SHPB). 

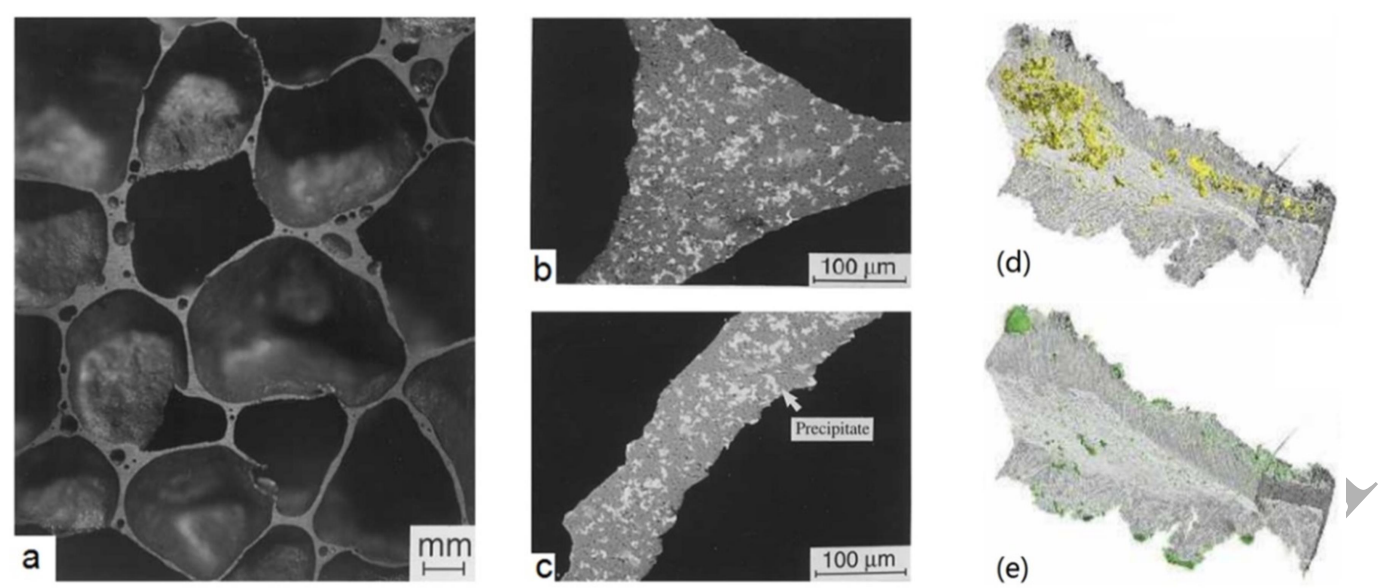

Fig. 14. Base material of closed-cell aluminium Alporas foam. Scanning electron micrographs (adapted from Ref. [12]): (a) cross-sectional structure of a collection of cells; (b) material at a junction node; (c) material at a cell face. Three-dimensional rendering of a cell wall $(\sim 1 \mathrm{~mm}$ long) from X-ray tomographic images (adapted from [187]); (d) micropores (highlighted in yellow); (e) coarse particles (highlighted in green).

Another issue is that the microscopic strain-rate experienced by the base material can be significantly different from the macroscopic strain-rate, because of localised cell deformation. An analysis of simple energy-absorbing structures is helpful to gain first-order understanding. Calladine and English [58] categorised energy-absorbing structures into two groups and investigated their collapse behaviour experimentally and theoretically. According to their definition, a Type I structure has a "flat-topped" compressive load-deflection curve while a Type II structure has a steeply falling one, as shown in Fig. 15. They found that the dynamic response of the Type II structure is more sensitive to impact velocity (accordingly, macroscopic strain-rate). According to their theoretical analysis, on one hand, the strain-rate at the locations of the plastic hinges of Type II structures is much larger than the macroscopic strain-rate (i.e. impact velocity divided by axial length); On the other hand, the transverse inertia (micro-inertia, see more discussion in Section 3.1.2) can retain the initial stage of dynamic deformation wherein axial compression dominates, which has a significant effect on the Type II structure. For the type I structure, the microscopic strain-rate can be smaller than the macroscopic strain-rate, and the micro-inertia has insignificant effect. 
(a)
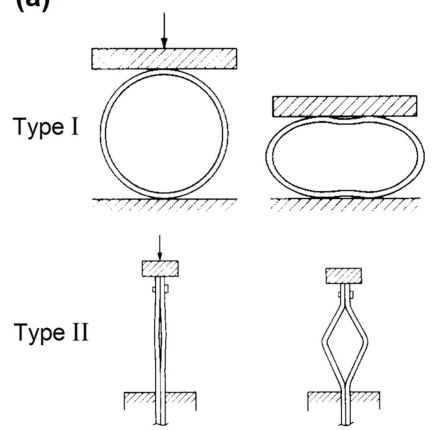

(b)

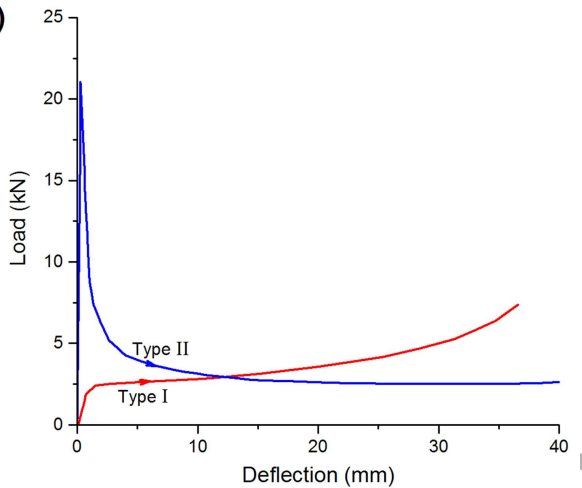

Fig. 15. Two types of energy-absorbing structures exhibiting distinctive compressive behaviours [58]: (a) examples of Type I and Type II structures; (b) the corresponding compressive load-deflection curves.

The above described distinctive behaviours of the Type I and Type II structures indicate that the macroscopically observed strain-rate effect is dependent on not only the microstructure of the base material (accordingly the rate dependence of base material properties), but also the meso-scale structural characteristics of the walls or struts of cells, which determine the base material deformation. Fig. 16 shows the microscopic strain distribution in the base material of a closed-cell polymer foam during dynamic compression, as well as the comparison between the microseopic strain-rate and macroscopic strain-rate [188]. It is clearly seen that microscopic strain-rate can be significantly larger than the macroscopic strain-rate. By contrast, according to the analytical estimate by Deshpande and Fleck [64], the maximum microscopic strain-rate in the base material of a cubic unit cell is approximately an order of magnitude lower than the macroscopic strain-rate, which seems only valid for certain types of cell geometries (e.g. the simplified cubic cell model in Ref. [64]). The discrepancy between the experimental observation and the analytical prediction by simplified model motivates the use of realistic meso-scale cell structures in numerical simulation, which can capture the heterogeneity of cell structure and deformation. The finite element modelling based on computed tomography, so-called image-based modelling [189] (see Section 4.1.2 for more details), is thus particularly useful to capture the dynamic compressive behaviour of a stochastic cellular material which may not be well described by regular cell model. 

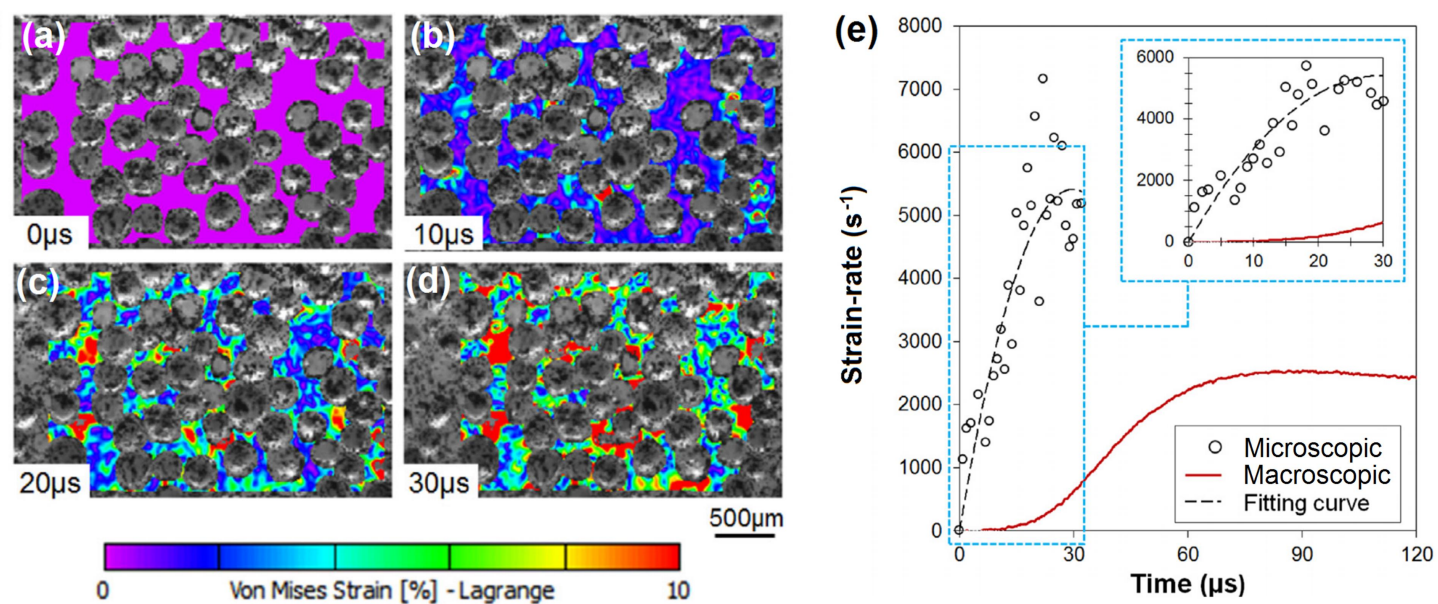

Fig. 16. Evolution of microscopic strain (von Mises effective strain) in the base material of a closed-cell PU-based polymeric foam at various time instants during the loading: (a) $0 \mu \mathrm{s}$ (macroscopic strain $=0 \%)$; (b) $10 \mu \mathrm{s}$ (macroscopic strain $=0.2 \%$ ); (c) $20 \mu \mathrm{s}$ (macroscopic strain $=0.6 \%)$; (d) $30 \mu \mathrm{s}$ (macroscopic strain $=1.8 \%$ ). The comparison between the microscopic strain-rate and macroscopic strain-rate is shown in (e). (Adapted from Ref. [188])

Recently, a 3D image-based FE simulation reveals that the rate dependence of the yield stress of base material is the main cause of the strain-rate hardening of the compressive strength of Alporas foam [26], as shown in Fig. 17. The compressive strength is independent of strain-rate in the transitional dynamic regime, if the base material is insensitive to strain-rate, which also indicates a negligible micro-inertia effect. For rate-dependent base material, the strain-rate sensitivity of the foam is slightly higher than that of the base material, which can be attributed to the microscopic strain-rate amplification [26, 188]. The experimentally observed rate dependence of Alporas foam has been captured by the $3 \mathrm{D}$ image-based modelling, see Fig. 17. However, the predicted magnitude of the rate dependence is overall lower than that observed in experiments, which may be attributed to the simplified Cowper-Symonds model used for the base material in modelling. The microstructure of base material in Alporas foam is complicated, see Fig. 14, and the exact rate dependence of the base material is still unclear. 


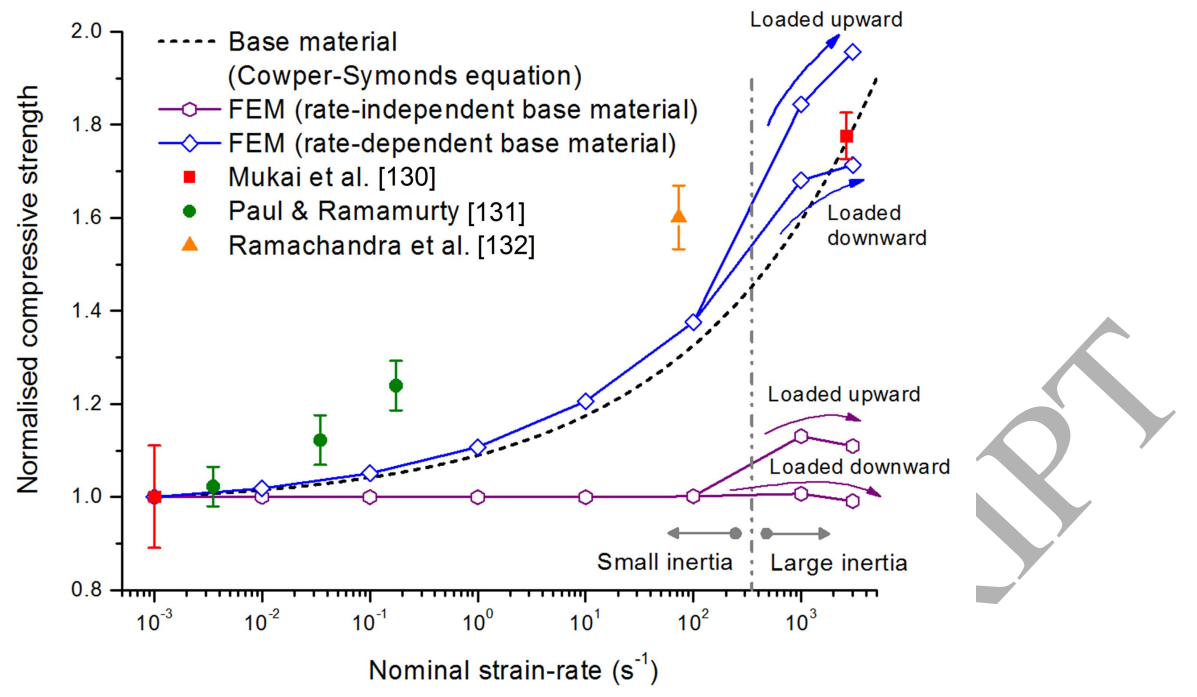

Fig. 17. Three-dimensional image-based modelling results [26] of the strain-rate sensitivity of the compressive strength (i.e. collapse initiation stress obtained at the supporting end) of closed-cell aluminium Alporas foam, alongside available test data [130-132].

The above conclusion is specific to Alporas foam. Another image-based simulation on open-cell aluminium m.pore ${ }^{\mathrm{TM}}$ foam reveals that both the rate dependence of base material and the micro-inertia contribute to the strain-rate hardening of the collapse stress in transitional dynamic regime [190], as shown in Fig. 18. However, the effect of the former outweighs the latter by a factor of 9.2 at a strain-rate of $100 \mathrm{~s}^{-1}$. The micro-inertia hardly affects the compressive stress after a strain of 0.01 , but the effect of the rate dependence of the yield stress of base material operates throughout the compression.

Mesoscopic simulations based on either idealised cells or actual cells [59, 70, 105] show that the effects of the rate dependence of base material properties and micro-inertia (discussed in Section 3.1.2) are sensitive to the characteristics of cell structure. For instance, the metallic Voronoi foams, both in 2D [59, 70] and 3D [105] tessellations, exhibit a lower strain-rate hardening than their base materials. According to Tagarielli et al.'s experimental observations [127], the R300, H130, HD130 and H250 PVC foams exhibit similar strain-rate sensitivity to the solid PVC, whereas the R75 and H100 PVC foams exhibit lower strain-rate sensitivity than the solid PVC. Therefore, it should be reiterated that the rate dependence of the base material can lead to increased, same or decreased strain-rate sensitivity of the cellular material, depending on the specific cell structure, and the associated microscopic deformation and strain-rates in the base material. Unfortunately, the characteristics of cell structures of foams may substantially differ among different manufacturing routes $[4,191]$, causing difficulty in 
making a general conclusion.
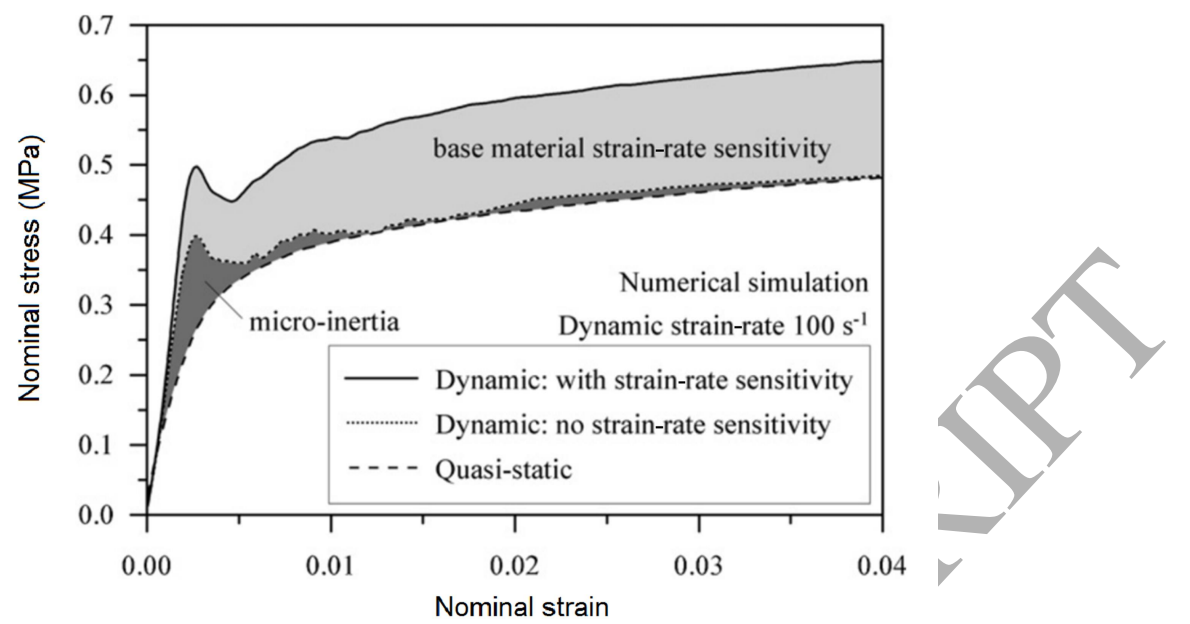

Fig. 18. Quasi-static and dynamic stress-strain curves obtained by image-based finite element modelling of the compression of an open-cell aluminium m.pore ${ }^{\mathrm{TM}}$ foam [190].

In shock compression, the deformation process becomes progressive cell crushing initiated from loading end (see Sections 2.3.1 and 3.2.1). It gives rise to an increased microscopic strain-rate within the shock front, which is much larger than the macroscopic strain-rate (note: nominal strain-rate under shock cannot be used in constitutive description), and the difference depends on the cell size and sample length. For base materials within the shock front, Radford et al. [147] argued that photon drags govern the plastic flow due to the high strain-rate and thus the stress is believed to be proportional to strain-rate due to the base material viscosity. However, it is still unclear about the quantitative relationship between the viscosity of the base material and that of the cellular material subjected to shock. In addition, a recent numerical study [157] indicates that the rate dependence of the yield stress of base material has significant effects on the shock properties, i.e. it enhances dynamic initiation stress, reduces shock strain and increases shock speed. However, the effect of the rate dependence of base material on the difference in the stress-strain relations of shock compression and quasi-static compression (Fig. 13) still require more experiments and simulations to clarify.

The foregoing discussion does not consider the effect of temperature on base material strain-rate sensitivity. On one hand, when environment temperature changes, the rate dependence of base material properties also changes [162, 176]. Indeed, Wang et al. [192] observed that the strain-rate sensitivity of aluminium foam increases as the temperature increases. On the other hand, the temperature of base material may rise when plastic work 
converts into heat. At high strain-rates it is expected that the heat transfer will be constrained and an adiabatic condition can be assumed [162]. The changing rate of base material temperature can be estimated by $\dot{T}=\beta \sigma \dot{\varepsilon} /\left(\rho_{\mathrm{s}} c\right)$ [26], where $\sigma, \dot{\varepsilon}, \rho_{\mathrm{s}}$ and $c$ are base-material stress, strain-rate, density and specific heat capacity, respectively, and $\beta$ is the Taylor-Quinney coefficient representing the fraction of plastic work converted into thermoplastic heating. For aluminium (and its alloy) base material, the typical parameter values are $\beta=0.9, \sigma=35.5-172 \mathrm{MPa}, \rho_{\mathrm{s}}=2.6-2.9 \mathrm{Mg} \cdot \mathrm{m}^{-3}$ and $c=920-1080 \mathrm{~J} \cdot \mathrm{kg}^{-1} \cdot \mathrm{K}^{-1}$ $[1,103,162,193]$. Then at a strain of 0.2 , the rise in temperature will be $2.0-12.9 \mathrm{~K}$ for a strain-rate of $1 \times 10^{3} \mathrm{~s}^{-1}$ and $2-129 \mathrm{~K}$ for a strain-rate of $1 \times 10^{4} \mathrm{~s}^{-1}$, which causes a thermal softening much less than that caused by the strain-rate hardening $[26,162,176]$.

\subsubsection{Micro-inertia}

Micro-inertia has been briefly discussed in Section 1.1. The two types of energy-absorbing structures proposed by Calladine and English [58], as shown in Fig. 15, have been extensively studied to elucidate the detailed mechanisms associated with micro-inertia (e.g. transverse inertia of material under macroscopic uniaxial deformation) [194-199]. These studies demonstrate a significant stress enhancement by micro-inertia for Type II structures. In modelling, the strain hardening and strain-rate sensitivity of the base material are usually disregarded, so as to simplify the problem and facilitate the analysis of micro-inertia effect. The impact simulations of the Type II structure composed of an elastic, perfectly plastic and rate-independent material [198] reveal that the larger the impact velocity is, the larger the load resistance will be, particularly for the initial response, as shown in Fig. 19, and the effect is more significant when the crookedness (Fig. 15) is smaller. However, it should be noted that the load enhancement effect of the micro-inertia almost vanishes when the displacement reaches a critical value corresponding to the occurrence of buckling. Indeed, the increase in the compressive load at early stage is because of the delay of the buckling by micro-inertia which prolongs the uniaxial compression but cannot avoid buckling. With the aid of strain hardening, micro-inertia can also increase the load resistance after the buckling, which will be discussed below. 


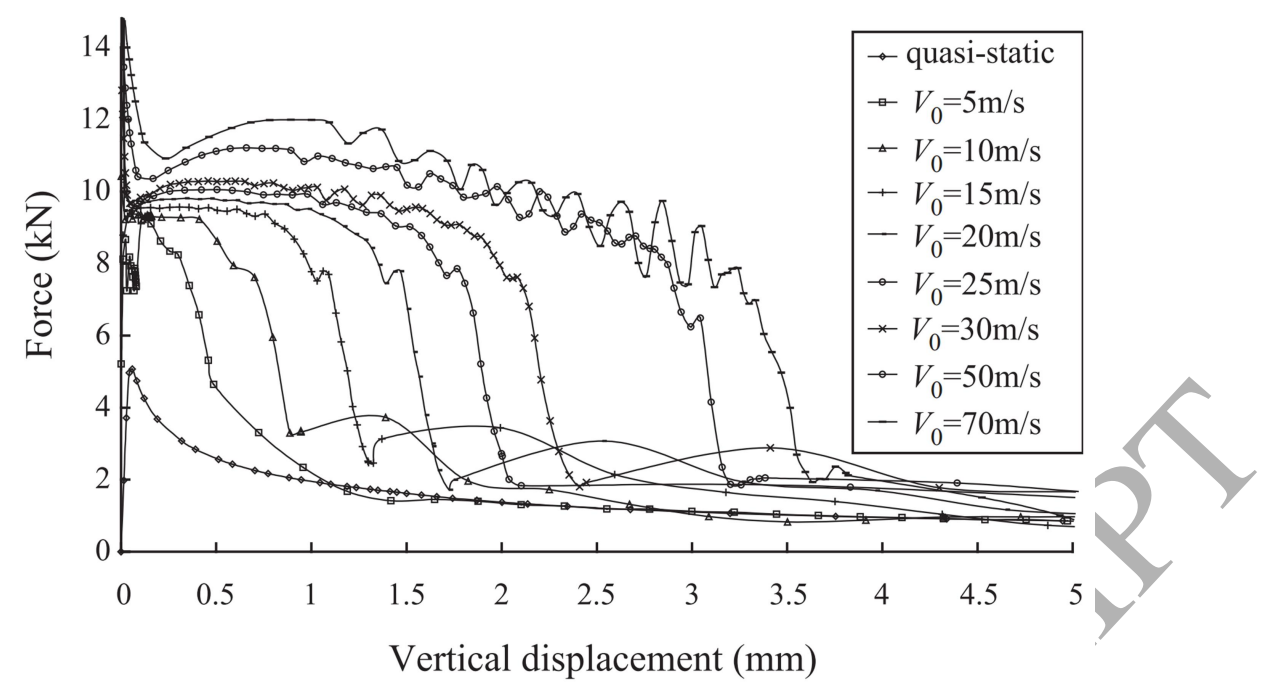

Fig. 19. Numerical results of axial force vs. vertical displacement when a Type II structure is subjected to impact with different initial velocity $V_{0}[198]$.

Many structures (e.g. tubes subjected to axial compression) can be categorised as Type II structures (Fig. 15). Karagiozova and Jones [50, 51] developed analytical and numerical models to study the effect of stress wave propagation and lateral inertia (i.e. micro-inertia) on the dynamic buckling of square tubes, and they found that the stress wave propagation in tube walls is strongly affected by material hardening properties, and the initiation of buckling is influenced significantly by elastic and plastic stress waves which propagate along the tube. They confirmed that the axial peak load can be enhanced by increasing impact velocity. Zhao and Abdennadher [63] experimentally investigated the axial impact response of a square tube made of strain-rate insensitive brass, with a primary aim to gain insights into the dynamic compressive behaviour of some cellular structures deformed by successive folding. They observed that both the initial peak force and subsequent crushing force were significantly increased in dynamic compression. Furthermore, they proposed a mechanism to explain the observed dynamic strength enhancement [63]: first, micro-inertia delays the buckling under impact loading and meanwhile leads to an increase in the strain of key load bearing elements; second, the stresses in these key elements are increased due to strain hardening, and thus, the crushing force increases as well, with loading continuing. This mechanism was confirmed by numerical simulation and corroborated by the postmortem micro-hardness measurements of the tested tubes.

Similarly, Hou et al. [48] compared the out-of-plane crushing behaviour of honeycombs made of two types of aluminium alloys with different strain hardening moduli, i.e. Al3003 (large hardening modulus) and A15052 (small hardening modulus), and observed that the 
dynamic crushing stress enhancement is much more significant for the honeycomb made of the aluminium alloy with a larger hardening modulus. For instance, for a relative density of $\sim 0.26$, the dynamic stress increase is $58 \%$ and $17 \%$ for the Al3003 honeycomb and Al5052 honeycomb, respectively. This can be explained by modelling results, as shown in Fig. 20, since the micro-inertia can delay structural buckling and produce larger stress in cell walls, due to strain hardening associated with axial compression of base material.
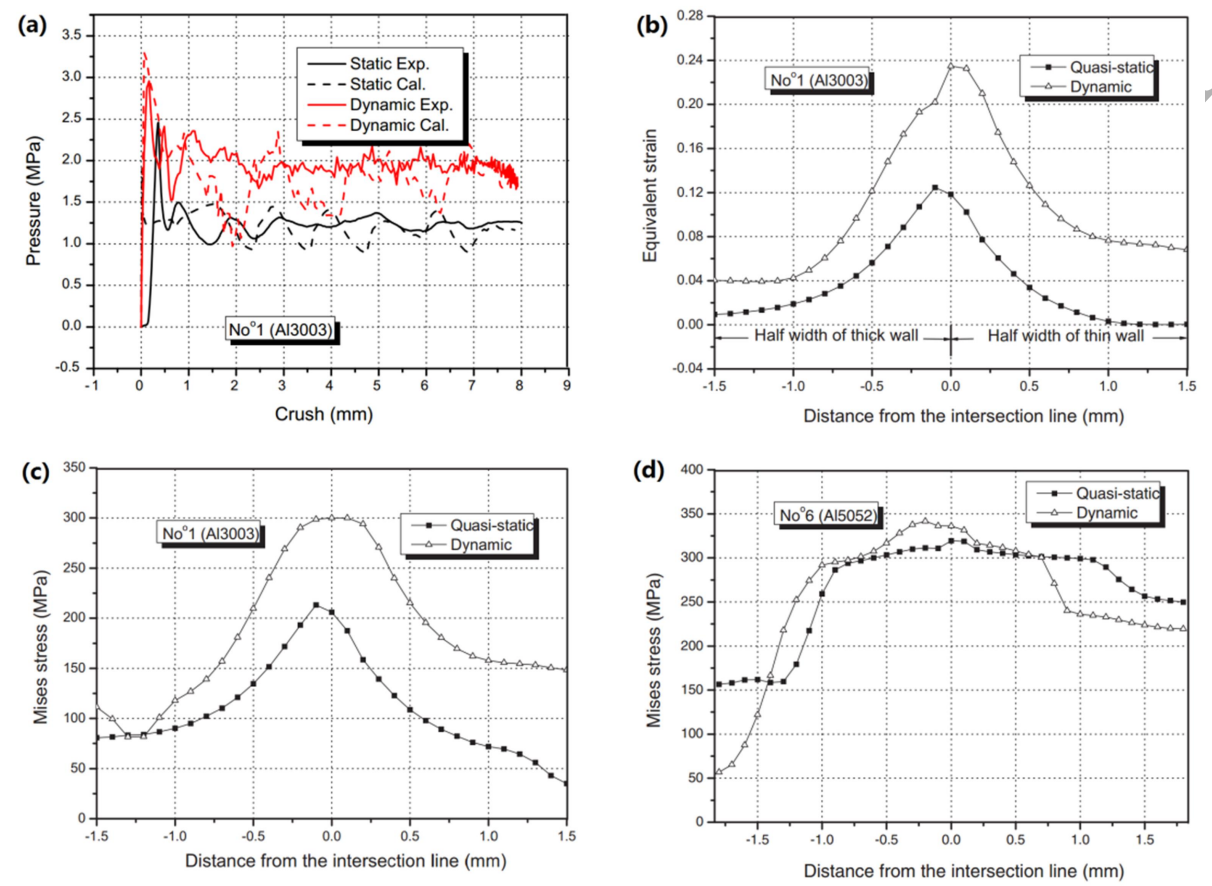

Fig. 20. Out-of-plane compression results of aluminium foams [48]: (1) comparison between experiment and simulation for Al3003 cell walls (large hardening modulus); (2) calculated equivalent plastic strain in A13003 cell walls; (3) calculated Mises stress in A13003 cell walls; (4) calculated Mises stress in A15052 cell walls (small hardening modulus).

So far, strong micro-inertia effect has been experimentally observed for cellular materials with cell walls/struts having small crookedness with respect to compression axis (e.g. honeycombs [48] and wood [154]). The underpinning mechanism is that the micro-inertia can restrain the lateral deformation of the cell wall, and thus, leads to a deformation mode which can resist a higher load. The micro-inertia alone can increase the peak stress or collapse stress in dynamic compression. This enhancement can be extended to the plateau stage by combining the micro-inertia effect and the strain hardening of the base material, as shown in Fig. 20. However, for foams, which have much more complicated orientation of cell 
walls/struts, many studies suggested that the micro-inertia effect is negligible or secondary, see Fig. 17 and Fig. 18.

To quantitatively evaluate the extent of micro-inertia in a complicated cell structure, Liu et al. [59] proposed a transverse-to-longitudinal acceleration ratio for FE simulations, viz.

$$
\begin{aligned}
& \mu_{X}=A_{X} / A_{Z} \\
& \mu_{Y}=A_{Y} / A_{Z}
\end{aligned}
$$

where $A=\left(\sum_{i=1}^{n} a_{i}^{2} / n\right)^{1 / 2}$ is the mean square root of the acceleration with $a_{i}$ being the acceleration of the $i$-th node in longitudinal loading direction ( $\mathrm{Z}$ axis) or transverse direction (X or Y axis) and $n$ being the total number of nodes on one side [59] or whole body [26] of a cellular structure. According to an analytical model by Zhang and $\mathrm{Yu}$ [197], this ratio is proportional to the square of the crookedness angle, and it is on the order of $10^{2}$ for a type II structure. However, this ratio is close to one for both Alporas foam (simulated by image-based model [26]) and 2D Voronoi foam [59]. Interestingly, this ratio decreases when strain-rate increases, implying that the contribution of micro-inertia effect is relatively weak for these foams even in the shock regime. In summary, the micro-inertia effect can be significant for out-of-plane compression of honeycombs and wood, but it is generally much less significant in foams.

\subsection{Meso-scale: cell structure}

\subsubsection{Distinctive cell deformations: energy minimisation vs inertial resistance}

In a cellular material subjected to uniaxial compression, the walls or struts of cells can be compressed (or stretched), bended or buckled. The actual deformation mode depends on the mesoscopic geometry and loading rate. It has been shown that topological criterion controls whether the mesoscopic deformation of a cellular material is bending-dominated or stretch-dominated in quasi-static loading regime [36, 37, 200]. In the transitional dynamic and shock regimes, micro-inertia restrains lateral motion of material points and hence it can delay buckling and promote uniaxial compression; macro-inertia can lead to the formation and propagation of a planar wave front, and thus, it produces more localised deformation associated with axial inertial force. Nevertheless, after global densification, the porosity is largely reduced, leading to the domination of the compression of the base material itself.

Two principles, i.e. energy minimisation under equilibrium and Newton's second law of motion, are important for the understanding of the deformation mechanisms at different 
temporal scales. First, in quasi-static and transitional dynamic regimes, the walls or struts of cells tend to deform in a way to minimise the internal energy of the whole cellular material system under force balance; when unstable deformation mode like buckling is involved, the micro-inertia could play an important role in the transitional dynamic regime, depending on whether the structure belongs to Type I or Type II. Second, in the shock regime, the base material responds to the macroscopic compressive load in a way controlled by the resistance of material points to the motion in the loading direction due to strong macro-inertia and some extent of micro-inertia; short-distance bending and buckling, as well as compression of cell walls/struts themselves, are localised at the loading end during shock propagation, and the principle of minimum energy for material under equilibrium does not work anymore.

For the cellular materials examined in this review, bending and buckling are the main deformation modes of cell struts/walls. Under quasi-static compression, in general, discrete or contiguous crush bands form and extend, and their locations and orientations are controlled by minimising the internal energy of the whole cellular material sample. For honeycombs, the deformation modes are substantially different between in-plane and out-of-plane compressions. The in-plane compression produces shear bands in the plateau stage, i.e. the cell walls bend elastically initially and then collapse by plastic bending [1, 66, 99, 201]. Outside the shear bands, most of cells remain their original shape. Under out-of-plane compression, the axial compression of the cell walls is dominant initially, followed by the cell-wall buckling when the critical load is attained [202,203]. The post-buckling response is localised, which is developed into first fold and subsequent progressive folding until the whole structure is completely folded and crushed. Wood exhibits similar out-of-plane compressive behaviour to honeycombs with respect to cell-wall buckling [204]. However, unlike honeycombs for which one cell extends throughout the out-of-plane axis, the cells (or grains) of wood are stacked in the axial direction, with a longitudinal-to-transvers length ratio being approximately 16:1 for balsa wood [92]. The two major deformation modes of wood under out-of-plane compression are buckling and kinking, accompanied by some shearing and lateral interaction between cell walls [92, 204].

Foams exhibit a deformation mode similar to the in-plane compression of honeycombs, despite the additional complexity due to their 3D cell structures with heterogeneous and stochastic characteristics. For closed-cell aluminium Alporas foam, Bastawros et al. [18] employed surface strain mapping method and X-ray computed tomography to characterise the local deformation in quasi-static compression. Their observations indicate that the Alporas foam deforms by the development of localised deformation bands whose width is 
approximately one-cell diameter, and the discrete deformation bands are developed one-by-one and are distributed at spacing of about 3-4 cell diameters. The plastic bending and buckling of cell walls are dominant during the collapse and crushing of cells. On cell level, the combination of distortion, rotation and shear occurs. For an open-cell aluminium foam, Zhou et al. [205] presented microscopic observation of dislocation slip bands in the struts of cells and the mesoscopic observation of the change of cell morphology, confirming the plastic bending and buckling.

Fig. 21 compares the compressive deformations of an open-cell aluminium foam in quasi-static, transitional dynamic and shock regimes $[69,206]$. It is evident that, in the quasi-static and transitional dynamic regimes, the first crush band forms in the presumably weakest sites and then more crush bands form in other sites which are separate from or adjacent to the first band. The weakest sites can be identified by examining the mesoscopic structural heterogeneity or imperfection [21, 207]. The localised deformation is randomly distributed in the sample. By contrast, in the shock regime, the crushed region appears at the loading end and then extends towards the other end of the sample by the propagation of the shock front. Experimental observations of shock deformation are also presented in Fig. 9 and Fig. 10 (see Section 2.3.1). This distinctive shock deformation arises from the material inertia in the loading direction (or macro-inertia), as the loading speed is too high to transmit the crushing load to the cells ahead of the shock front. 

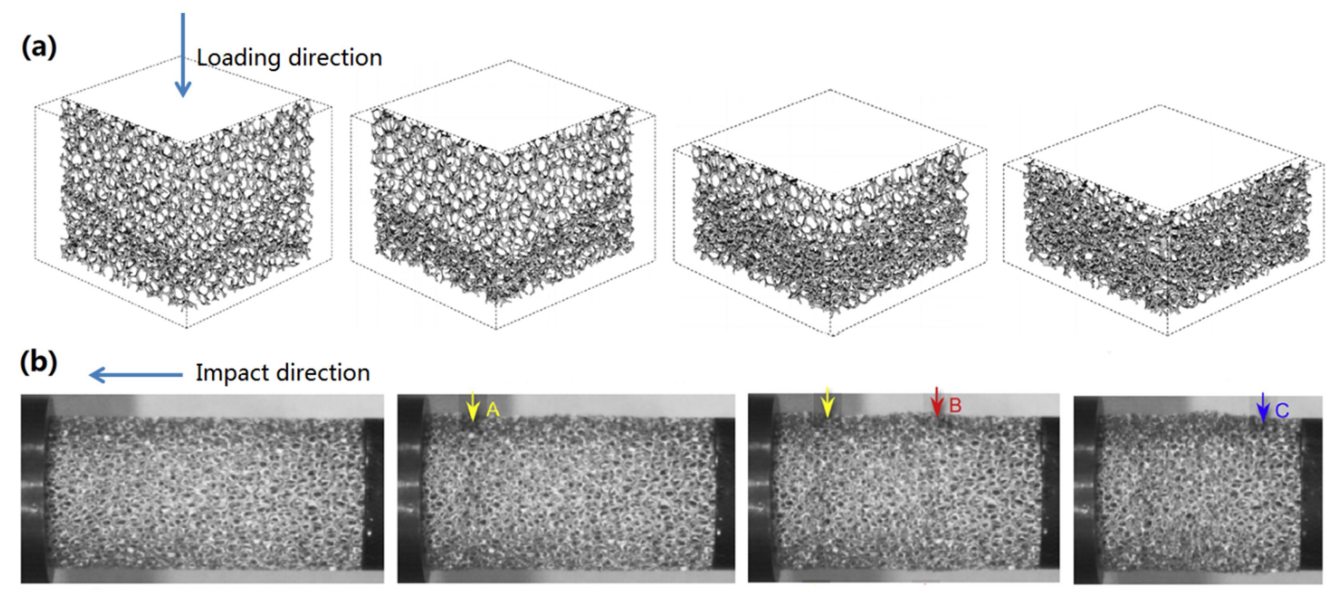

(c) $\longleftarrow$ Impact direction
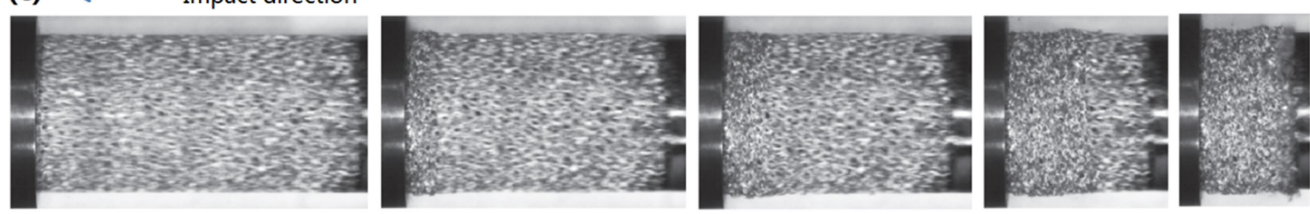

Compression towards global densification

Fig. 21. Sequence of compressive deformation of open-cell aluminium Duocel foam: (a) quasi-static compression (adapted from Ref. [206]); (b) transitional dynamic compression (adapted from Ref. [69] and note that discrete crush bands are indicated by letters A-C); (c) shock (adapted form Ref. [69]), also see DIC results in Fig. 10a. Note that the dynamic test employed the direct impact setup, as shown in Table 1.

The shock deformation has significant implications in energy absorption. An archived discussion between Tan et al. [208] and Fleck and Deshpande [209] showed their different understandings on this issue. Tan et al. [208] emphasised the mesoscopic structural causes of the shock enhancement of energy absorption and they demonstrated that the energy absorption significantly increases with impact velocity due to different deformation modes developed, according to their compression simulation results for a honeycomb with a rate-independent base material. By contrast, Fleck and Deshpande [209] approximated the dynamic energy absorption to be the same as the quasi-static energy absorption in a structural analysis, and they argued that a switch of mesoscopic deformation mode from bending to stretching cannot explain the increased energy demonstrated by Tan et al. [208]. In another paper co-authored by Fleck and Deshpande [147], they discussed the contribution of the intrinsic viscosity (e.g. photon drag) of cell walls to the additional energy dissipation in the shock of an aluminium foam. Zou et al. [20] argued that the shock enhancement of energy dissipation is mainly due to the increased local plastic bending and axial compression strains 
in the inclined cell walls, which agrees with Tan et al.'s opinion [208], and they emphasised the roles of inertial resistance in the development of such localised deformation modes. Recently, Sun et al. [25] presented the microscopic deformation in the base material of a 2D image-based foam and demonstrated that the mesoscopic structural cause of the additional energy dissipation can be the increase of plastic hinges in the cell walls under shock, as shown in Fig. 22, rather than switching the main deformation mode of cell walls from bending to stretch/contraction. However, the viscosity of base material is excluded in the model in Ref. [25], which may also contribute to the additional energy dissipation in shock compression of some actual foams in experiments.

(a)
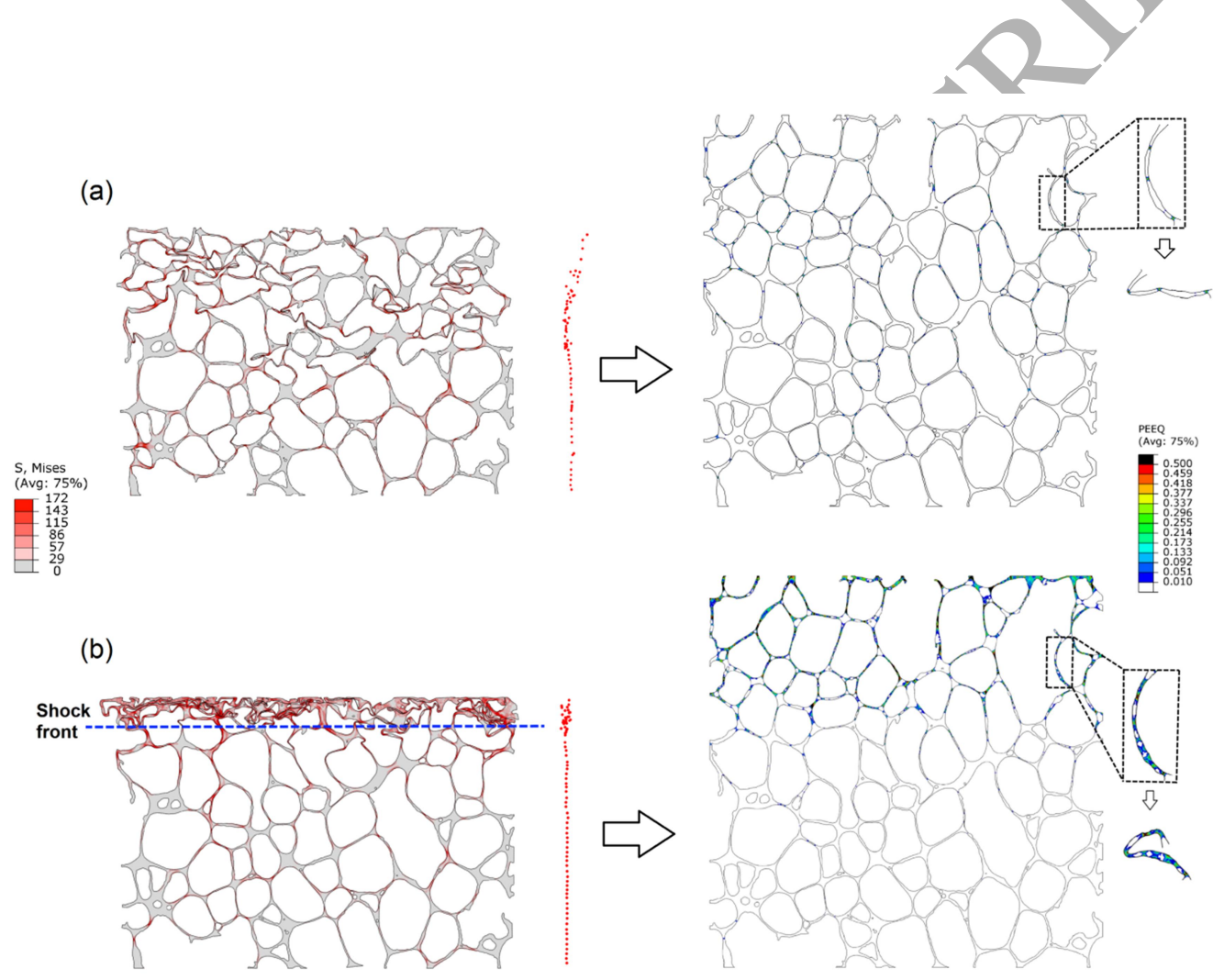

(b)

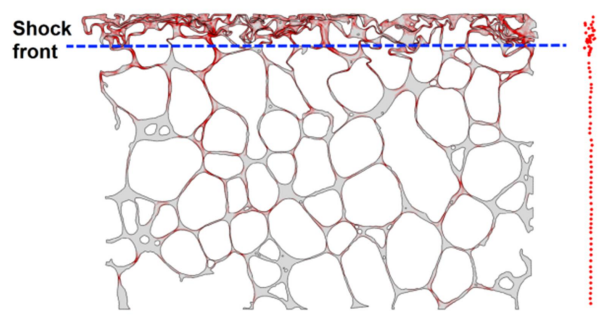

Fig. 22. Compressive deformation of a 2D image-based foam at a nominal strain of 0.3 [25]: (a) quasi-static compression; (b) shock. The arrayed 1D "particle" represents the average motion of each cross-section. Note that the Mises stress distributions in the deformed cell walls are shown by the colour scale (left); whereas the cell-wall strain contours (right) are plotted in the original reference configurations, and the enlarged views show the details of the strain distribution in one cell wall.

It has been mentioned in Section 2.3.1 that "shock enhancement" is commonly interpreted as an increase in crushing stress or energy absorption in early studies. Recently, this concept has been extended to strain, thanks to the development of local strain characterisation and 
measurement methods $[13,20,22,23,25,69,149,158,210]$. In fact, the "shock enhancement" arises from the jump of these physical quantities across shock front. The higher the impact velocity is, the larger the jump is. Recalling the stress-strain relations shown in Fig. 13, it is interesting to see that the shock stress is actually smaller than the quasi-static stress at the same strain in the densification stage, i.e. the locus of shock stress-strain states is below the quasi-static stress-strain curve. However, it should be noted that the base materials (aluminium alloys) of the foams tested in Fig. 13 were reported to be strain-rate insensitive $[69,149]$. If the base material is strain-rate sensitive, the locus of shock stress-strain states may appear above the quasi-static stress-strain curve, in contrast to those shown in Fig. 13. Indeed, for a polymer foam, it has been experimentally observed that the shock stress is larger than the quasi-static stress at the same strain in the densification stage [161]. It is generally agreed that the difference between the locus of shock stress-strain states and the quasi-static stress-strain relation is caused by the distinctive cell deformations under these two different loading conditions.

\subsubsection{Gas pressurisation}

In 1966, Shaw and Sata [61] observed that the compressive stress of a polystyrene foam increased with strain-rate and they surmised that the strain-rate hardening could result from the increased resistance to gas flow within the cell structure at high strain-rates. Subsequently, many researchers believed that the pressurisation of the internal gas enclosed by the cells of a cellular material can give rise to a strain-rate hardening [29, 141, 143, 211]. Despite being invoked to explain dynamic experimental observations, gas effect in closed-cell foams was not distinguished between quasi-static and dynamic compressions in most of previous theoretical and numerical models [1, 212-216], since it was assumed that the internal gas cannot escape from the closed cells and thus the gas pressurisation is strain-rate independent. Moreover, an arbitrary value of the initial pressure of enclosed gas is often assumed in theoretical and numerical studies on gas effect [212-215], while in reality, the enclosed gas in man-made cellular materials usually has a pressure close to ambient pressure before loading [1].

The assumption that gas effect is rate independent is actually questionable. For many closed-cell foams, the cell walls are not perfectly "closed" in the compression process due to either fabrication defects or cell-wall rupture caused by crushing. Thin membranes are prevalent in a closed-cell foam, resulting in significant reduction of the bulk density, but increasing the chance of cell cracking during compression, unlike a porous material in which 
the pores are typically isolated in the matrix material. Compared to open-cell foams, closed-cell foams can only provide limited, relatively small "channels" for gas flow and hence the gas pressurisation is more sensitive to the time allowed for the gas flow which is controlled by loading rate. With extensive channels for gas flow, open-cell foams are usually not strengthened by internal gas, unless the cell size is extremely small (e.g. below $20 \mu \mathrm{m}$ ) [1].

Bouix et al. [29] provided direct experimental evidence to support above argument. They observed that the gas flow (indicated by bubbles in a water chamber) in a closed-céll polypropylene foam can be significantly reduced when the compressive strain-rate increases, as shown in Fig. 23. It demonstrated that a full gas flow occurred throughout the quasi-static compression, but in dynamic compression only little gas escaped and the internal gas was effectively trapped in the crushed regions. Since large gas pressure can be produced due to the trapping of gas in a dynamic compression process, it may be expected that the dynamic compressive stress will be larger than the quasi-static stress due to the contribution of gas pressure.

Strain-rate $=0.01 \mathrm{~s}^{-1}$
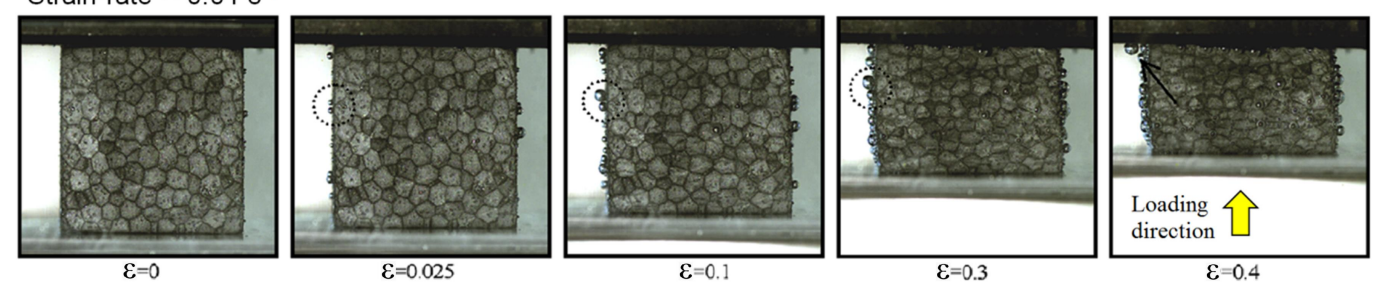

Strain-rate $=200 \mathrm{~s}^{-1}$
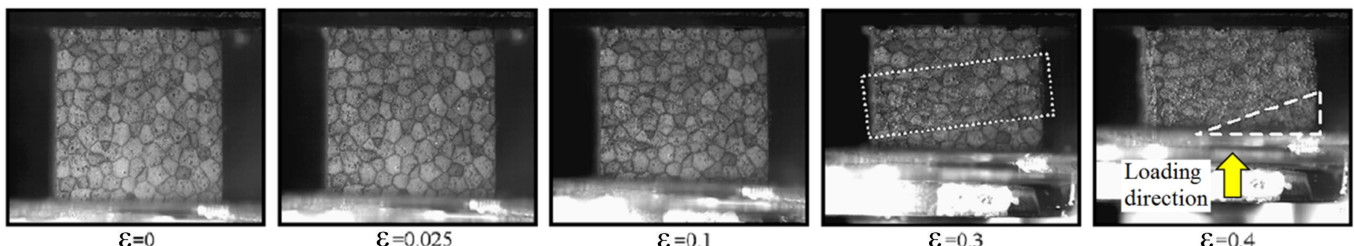

Strain-rate $=1500 \mathrm{~s}^{-1}$
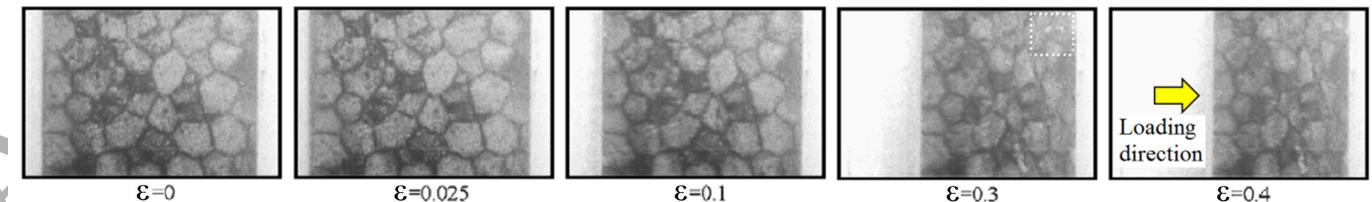

Fig. 23. Gas flow during quasi-static and dynamic compressions of closed-cell EPP foam in water at nominal strain-rates of (a) $0.01 \mathrm{~s}^{-1}$, (b) $200 \mathrm{~s}^{-1}$ and (c) $1500 \mathrm{~s}^{-1}$ (adapted from Ref. [29]). The gas flow is visualised by bubbles generated by the gas escaped from the foam.

Other indirect experimental evidences have been also reported. For instance, Zhang and 
$\mathrm{Yu}$ [217] elucidated two mechanisms to account for the enhancement of load resistance of pressurised thin-walled circular tubes, i.e. a direct effect of the internal air pressure and an indirect effect associated with the gas-solid interaction. More relevantly, Xu et al. [138] tested the dynamic out-of-plane compression of aluminium foams with different hole percentages on the sealed sample ends, as shown in Fig. 24. They found that the entrapped air hardly affects the stress at the early phase of the plateau stage but leads to a significant strain hardening near the onset of densification. These specially designed tests on regularly shaped/arrayed cell structures provide valuable insights into the underpinning mechanism of gas effeet when cell deformation is uniform (hence uniform gas pressure), but they have limitation to explain the gas effect in foams which have more complicated cell structures and deformations (see Section 3.2.1).

(a)
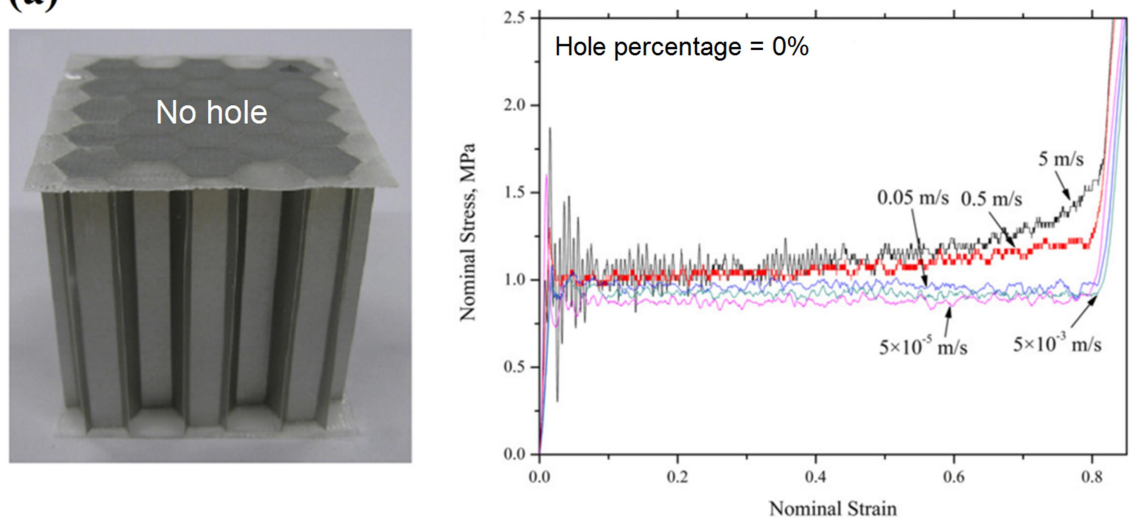

(b)
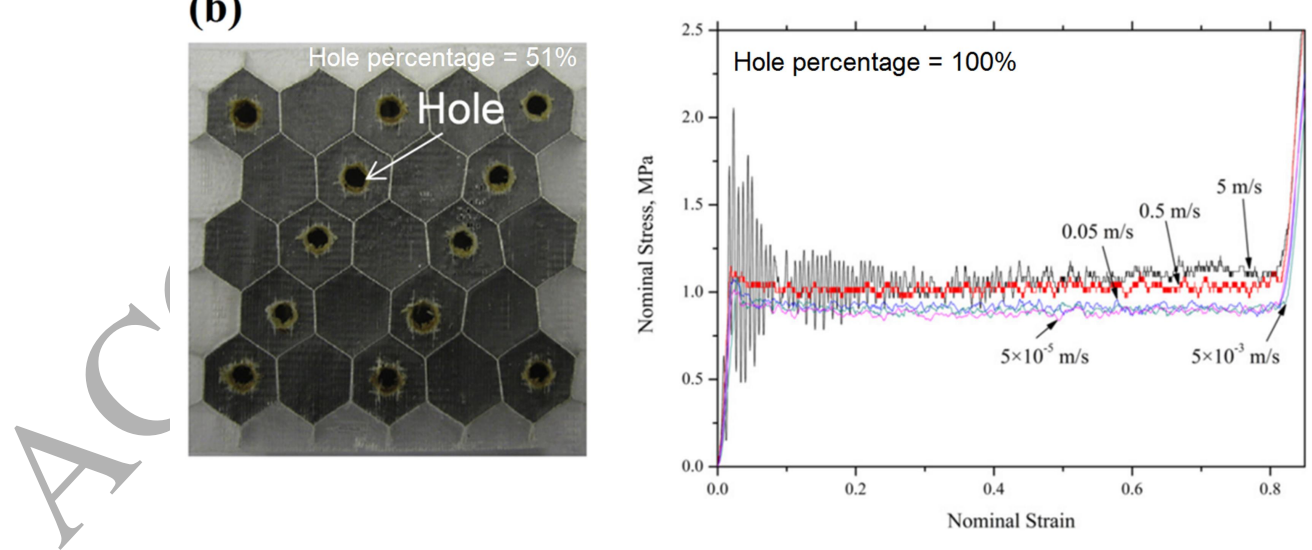

Fig. 24. Compressive stress-strain curves of an aluminium honeycomb at different out-of-plane loading velocities ranging from $5 \times 10^{-5}$ to $5 \mathrm{~m} / \mathrm{s}$ with a hole percentage of: (a) $0 \%$; (b) $100 \%$. (adapted from Ref. [138]) 
The direct measurement of the gas pressure in each cell of a closed-cell foam during compression is obviously difficult if not impossible. Sadot et al. [218] employed a novel experimental setup using a folded paper enclosing trapped air and a shock tube to mimic the gas response in the cells of a closed-cell aluminium foam, as shown in Fig. 25. They obtained the variation of gas pressure with strain and found that the gas pressure is the main cause of the enhanced stress at high strain-rates for the light-weight foam with a low relative density (i.e. $4 \%)$.
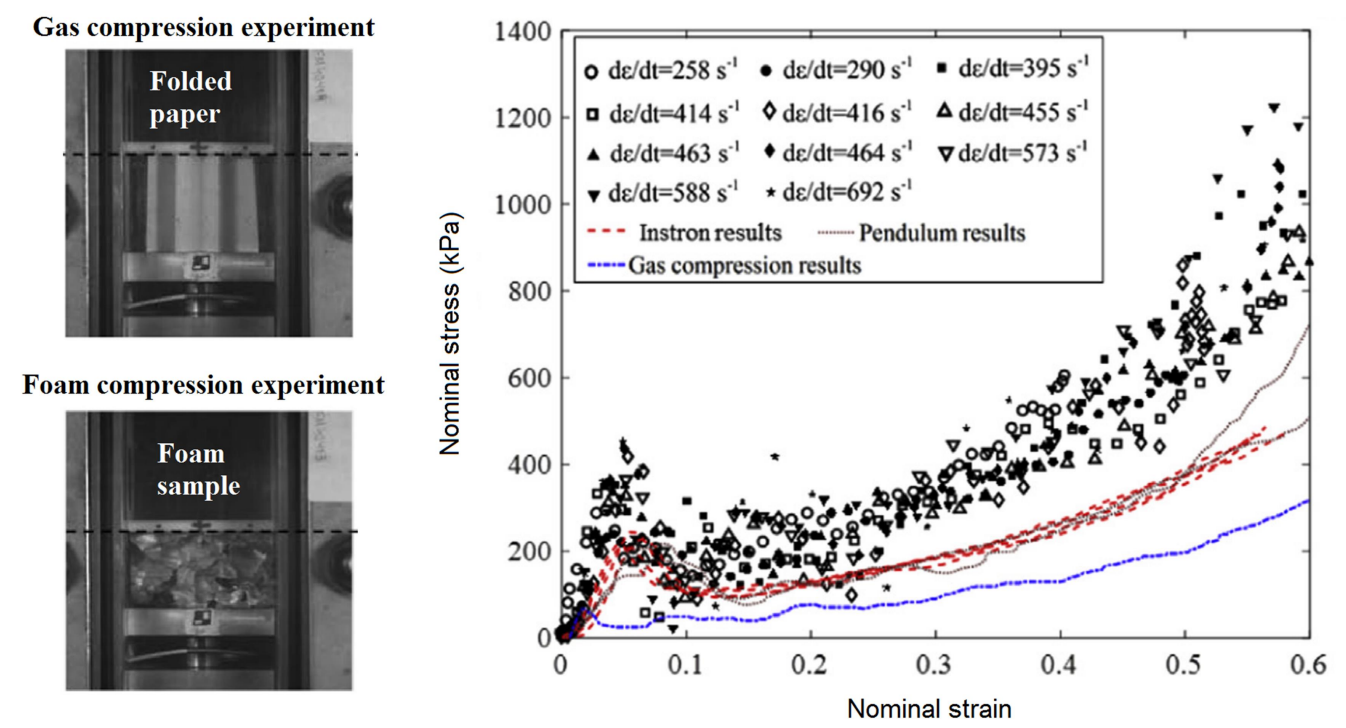

Fig. 25. Compressive stress-strain results for the closed-cell aluminium Cymat foam at different strain-rates and the entrapped gas in a folded paper under high speed impact (adapted from Ref. [218]).

These experimental studies demonstrated the entrapped gas effect, and support the consideration of gas enhancement of dynamic compressive stress, particularly for closed-cell foams having relatively low strength. To investigate the mesoscopic mechanism, numerical models capable of capturing nonuniform cell deformation and higher loading rates (e.g. shock regime) are needed. Banerjee and Bhawalkar [219] first presented modelling results of the gas-solid interaction within a random cell structure of a metallic foam compressed at different loading speeds $(20-200 \mathrm{~m} / \mathrm{s})$. They found a clear gas effect on the stress-strain curves but did not provide further analysis. Sun and Li [34] carried out a more thorough qualitative analysis of the mesoscopic mechanism of gas effect on dynamic compressive stress and deformation of cellular solids with different cell morphologies and loading rates, based on 2D mesoscopic finite element models. The main findings are briefly summarised below. 
The modelling results showed that the entrapped gas affects the cell deformation during the compression, leading to less compaction in the crush band due to the gas pressurisation in the cells. The locally high gas pressure in the crush band, however, hardly affects the nominal stress in the plateau stage. This is because the plateau stress is determined by the sequential formation of different crush bands, outside which the cells remain their original volumes and thus the gas pressure is actually collectively too small to enhance the global load resistance until the global densification begins. This mechanism is consistent with the numerical results obtained from another 3D cell-based model [220]. Equally importantly, the entrapped gas can reduce the densification initiation strain (see Fig. 26a) determined from the stress-strain curve, and similar reduction in densification initiation strain has been also observed in dynamic compression test of closed-cell Cymat foam (see Fig. 26b). When shock is initiated (e.g. $\dot{\varepsilon}=1000 \mathrm{~s}^{-1}$ or $V_{\mathrm{i}}=42 \mathrm{~m} / \mathrm{s}$ ), the strain-rate experienced by the crushed cells is significantly larger than the nominal strain-rate, and the gas pressure, as well as the nominal stress, can rise extremely fast. This will produce a pulse-like stress feature when the densification stage is approached (Fig. 27a), and similar pulse-like increase in stress has been observed in experiments by Merrett et al. [126], see Fig. 27b. Interestingly, the amplitude of the experimentally observed short pulse of crushing stress increased with the increase in impact velocity, as shown in Fig. 27b, which may imply that the higher-speed impact can more effectively trap gas and thus produce larger gas pressure to enhance the crushing stress. 

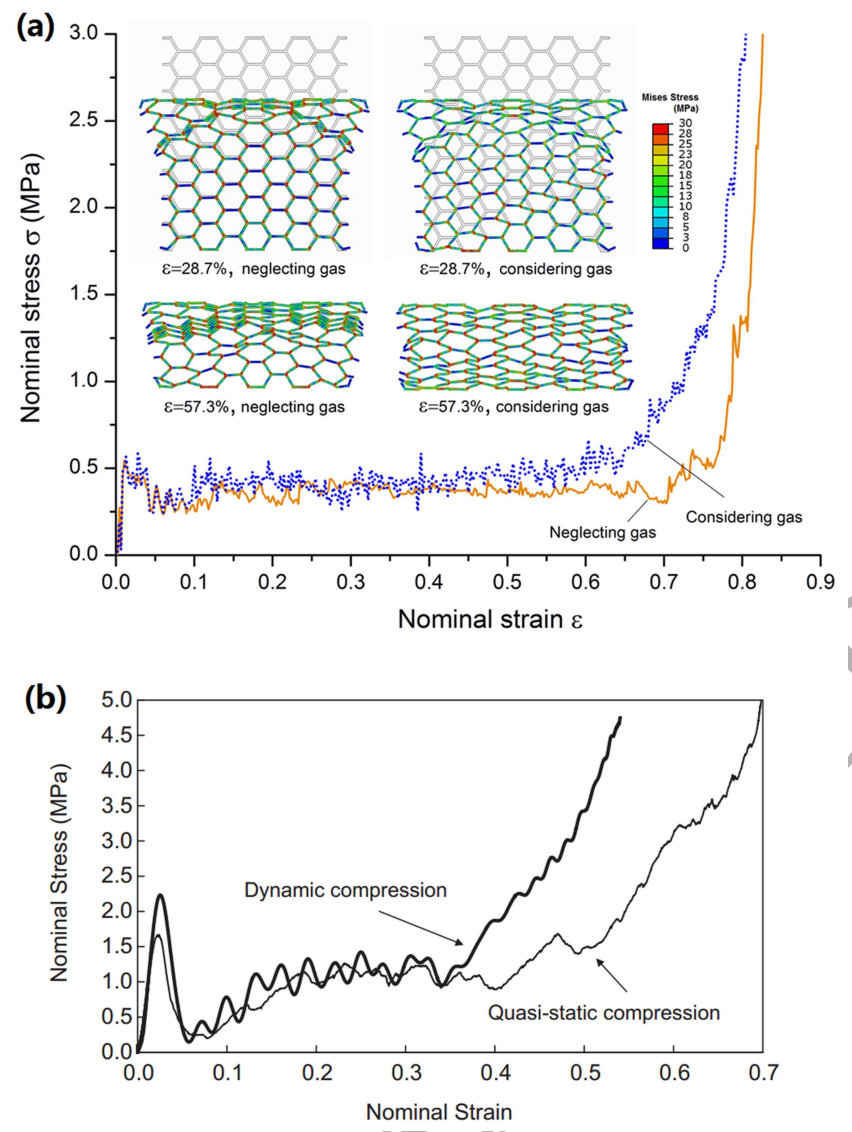

Fig. 26. (a) Simulation of the dynamic crushing of a hexagonal-cell structure at a nominal strain-rate of $100 \mathrm{~s}^{-1}$ [34]; (b) experimental comparison of the quasi-static stress-strain curve and the dynamic stress-strain curve of Cymat foam at a strain-rate of $100 \mathrm{~s}^{-1}$ [115]. 

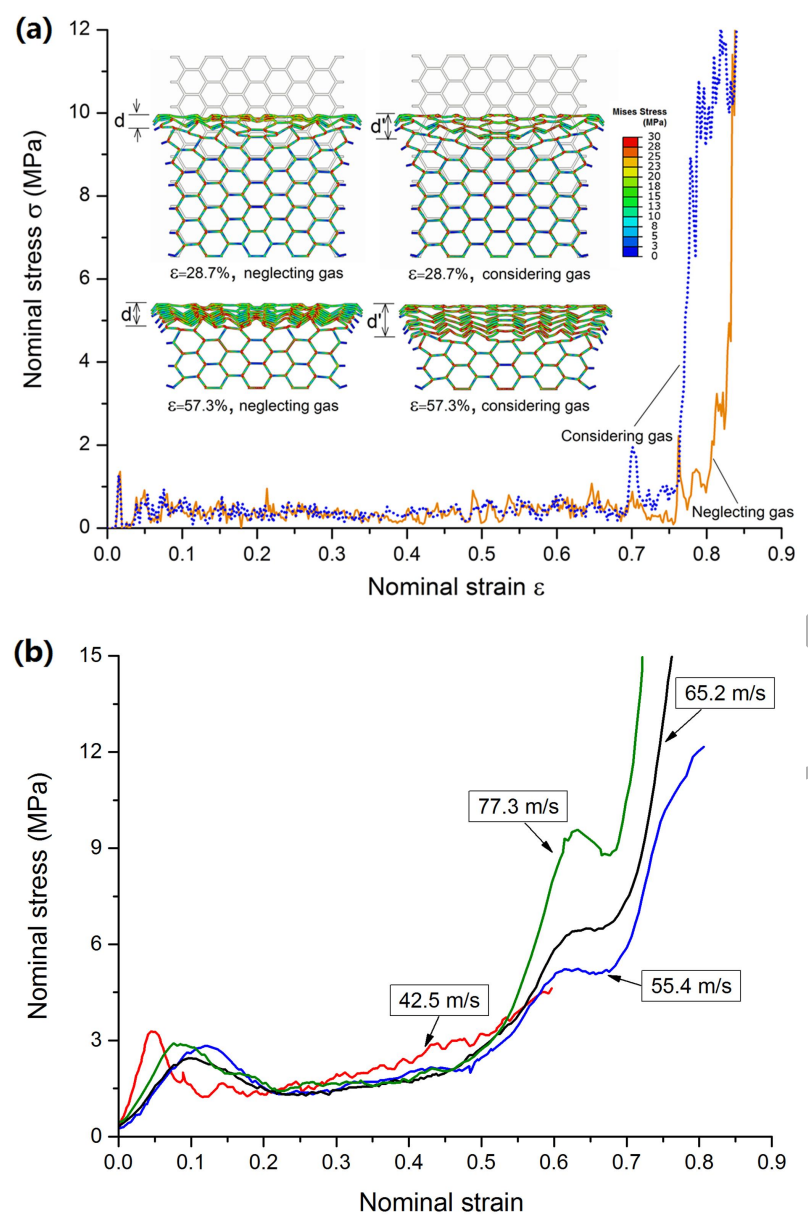

Fig. 27. (a) Simulation of the dynamic crushing of a hexagonal-cell structure at a nominal strain-rate of $1000 \mathrm{~s}^{-1}$ (impact velocity: $42 \mathrm{~m} / \mathrm{s}$ ) [34] (note: the stress scale in Ref. [34] is adjusted here); (b) experimental comparison of the dynamic compressive stress-strain curves of Alporas foam under different impact velocities [126]. The nominal stresses are obtained on the supporting end.

Gibson and Ashby [1] proposed an formula to estimate the gas-induced stress enhancement, which was recently modified in Ref. [34] to incorporate higher-order strain terms. Two thermodynamic processes for an ideal gas, i.e. isothermal and adiabatic processes, are assumed in the theoretical approach. The adiabatic assumption has been experimentally proved valid at high strain-rates [218]. However, it may not be straightforward to define the critical strain-rates for different thermodynamic processes. A cubic cell model was used in Ref. [34] to determine the critical strain-rate $\dot{\varepsilon}_{\mathrm{a}-\mathrm{c}}$ for an adiabatic process and then the critical strain-rate $\dot{\varepsilon}_{\mathrm{i}-\mathrm{c}}$ for an isothermal process can be conservatively assumed smaller than 
$\dot{\varepsilon}_{\text {a-c }}$ by two orders of magnitude. First, the below ratio of heat transfer rate to power is established

$$
\frac{\dot{Q}}{\dot{W}}=\frac{H T_{0}[6-4(1-2 v) \varepsilon]\left\{[1-(1-2 v) \varepsilon]^{1-\gamma}-1\right\}}{p_{0} l_{0}(1-2 v)[1-(1-2 v) \varepsilon]^{-\gamma}}\left(\frac{1}{\dot{\varepsilon}}\right)
$$

where $\dot{Q}$ is the heat transfer rate, $\dot{W}$ is the power, $\varepsilon$ is the engineering compressive strain, $v$ and $l_{0}$ are the Poisson's ratio and the internal edge length of the cubic cell, respectively, $\gamma$ is the ratio of the specific heat capacities, $p_{0}$ and $T_{0}$ are the initial gas pressure and temperature, respectively, and $H$ is the heat transfer coefficient. If this $\dot{Q} / W$ ratio is sufficiently small, the heat transfer can be neglected and then the process can be regarded as adiabatic. For typical parameters, $\dot{\varepsilon}_{\mathrm{a}-\mathrm{c}}=10^{2} \mathrm{~s}^{-1}$ and $\dot{\varepsilon}_{\mathrm{i}-\mathrm{c}}=10^{0} \mathrm{~s}^{-1} \quad$ [34].

When the higher-order strain terms are considered, the stress enhancement due to entrapped gas can be expressed as

$$
\Delta \sigma=\frac{1-(1-\varepsilon)(1+v \varepsilon)^{2}}{(1-\varepsilon)(1+v \varepsilon)^{2}-\rho / \rho_{\mathrm{s}}} p_{0}
$$

for an isothermal process $\left(\dot{\varepsilon}<\dot{\varepsilon}_{\mathrm{i}-\mathrm{c}}\right)$, and

$$
\Delta \sigma=\left[\left(\frac{1-\rho / \rho_{\mathrm{s}}}{(1-\varepsilon)(1+v \varepsilon)^{2}-\rho / \rho_{\mathrm{s}}}\right)^{\gamma}-1\right] p_{0}
$$

for an adiabatic process $\left(\dot{\varepsilon} \geq \dot{\varepsilon}_{\mathrm{a}-\mathrm{d}}\right)$, where $\rho / \rho_{\mathrm{s}}$ is relative density. The 2D version of Eqs. (15) and (16) has been shown in Ref. [34]. For the strain-rates between these two extreme cases $\left(\dot{\varepsilon}_{\mathrm{i}-\mathrm{c}}<\dot{\varepsilon}<\dot{\varepsilon}_{\mathrm{a}-\mathrm{c}}\right)$, the temperature effect can be accounted by a linear interpolation, leading to following stress enhancement equation

$$
\Delta \sigma=\left\{\frac{1-\rho / \rho_{\mathrm{s}}}{(1-\varepsilon)(1+v \varepsilon)^{2}-\rho / \rho_{\mathrm{s}}}\left[\left(\frac{\dot{\varepsilon}_{\mathrm{a}-\mathrm{c}}-\dot{\varepsilon}}{\dot{\varepsilon}_{\mathrm{a}-\mathrm{c}}-\dot{\varepsilon}_{\mathrm{i}-\mathrm{c}}}\right)+\left(\frac{\dot{\varepsilon}-\dot{\varepsilon}_{\mathrm{i}-\mathrm{c}}}{\dot{\varepsilon}_{\mathrm{a}-\mathrm{c}}-\dot{\varepsilon}_{\mathrm{i}-\mathrm{c}}}\right)\left(\frac{1-\rho / \rho_{\mathrm{s}}}{(1-\varepsilon)(1+v \varepsilon)^{2}-\rho / \rho_{\mathrm{s}}}\right)^{\gamma-1}\right]-1\right\} p_{0}
$$

Above equations assume that the gas pressure is uniform and the stress enhancement is equal to gas pressure. Eqs.(15) and (16) are equivalent to Gibson-Ashby equations when the Poisson's ratio is equal to zero, but in other cases their predictions are considerably different from those predicted by Gibson-Ashby equations $[1,34]$. Indeed, the analytical estimate using Gibson-Ashby equations [1] leads to conclusions that the gas effect in metal foams is negligible even at densification initiation strain $[59,64,65]$, whereas the above equations can predict much higher stress enhancement at large strains depending on Poisson's ratio [34]. In general, these equations show that stress enhancement is larger when the relative density and 
Poisson's ratio are decreased, and an adiabatic process in a dynamic compression leads to higher stress enhancement than an isothermal process.

One limitation of these equations is that they are essentially rate-independent unless heat transfer effect is approximately taken into account, see Eq. (17). In fact, a free gas flow and a complete gas trap are two extreme cases corresponding to sufficiently slow and fast compressions, respectively. In intermediate loading cases, the gas leaking rate may need consideration. $\mathrm{Xu}$ et al. [138] proposed the below equation to define the gas leak for an isothermal process

$$
\delta=1-\frac{p V}{p_{0} V_{0}}
$$

where $V_{0}$ and $V$ are the initial and current volumes of the gas, respectively. They further derived the stress enhancement by neglecting lateral deformation and using a first-order approximation, and the derived equation is expressed as

$$
\Delta \sigma=p_{0}\left(\frac{1}{1-\varepsilon}-1\right)\left(1-\frac{\dot{\delta}}{\dot{\varepsilon}}\right)
$$

It is seen that both gas leaking rate and strain-rate are incorporated in above equation which has been applied to analyse the gas effects in aluminium honeycombs [138] and foams [108]. In practice, however, the gas leaking rate is difficult to measure and thus this parameter may need calibration from case to case. Xu et al. [138] fit their experimental data of aluminium honeycombs and found that the gas leaking rate $\dot{\delta}$ is dependent on the strain-rate and the percentage of holes on sample ends, but independent of the cell size and the ratio of cell wall thickness to edge length. Furthermore, in some cases, gas leak is also associated with the cracking of cell walls and thus dependent on the compressive strain. For instance, the base material of composite metal foam exhibits one cracking mode under quasi-static compression but two cracking modes under dynamic compression [221]; this difference is believed to enable the dynamic compression to effectively trap the internal gas at strains below $20-30 \%$, but the gas leaks rapidly afterward.

The analytical predictions from Eqs. (15)-(19) have reasonable accuracy in the transitional dynamic regime when the compression is approaching to and entering the densification stage wherein the cell deformation is relatively uniform $[34,138]$. But they are not accurate when localised cell crushing occurs in shock, and in such a case numerical simulations considering nonuniform gas pressure and gas-solid interaction for different cell structures may be needed to capture the gas-induced stress enhancement [34]. Recent numerical studies [34, 157, 220] have provided important insights into the mesoscopic mechanism of the gas effect. However, 
some issues are still open to research, e.g. quantification of the gas leak or gas flow in different compression stages, and the implication of gas dynamics in the dynamic compression of cellular materials.

Above discussion has shown that the gas effect is associated with thermal process, deformation mode and gas leaking. If the gas can be completely trapped in cells with significant compaction, the contribution of gas pressurisation to crushing stress will be comparable to the solid structure. Otherwise, gas effect will be less considerable except for cellular materials having very low strength (or density), since limited gas pressure can be produced due to either less compaction of cells or higher gas leaking rate during compression. In general, cell morphology can affect gas pressurisation via its effect on cell compaction [34]. Other structural parameters such as relative density and cell size may also play a role, requiring more experimental and numerical evidences. Besides the gas effect on stress, it was also found that the densification initiation strain (defined using energy absorption method, see Section 2.1.2) can be reduced by gas pressurisation and this reduction is dependent on strain-rate and cell morphology [34]. Entrapped gas can also increase the shock speed and affect the shock stress-strain state [157]. Furthermore, the gas pressurisation may have significant implication in energy absorption, which may be worth exploring in future research.

\section{Modelling}

\subsection{Meso-scale models}

\subsubsection{Mass-spring models}

Meso-scale models can take into account the effects of cell structures either implicitly or explicitly. Their values are threefold: first, they can be used to examine and quantify the mechanisms discussed in Section 3; second, they are alternative to experiments for identifying the sensitivity of compressive properties to different factors via parametric sensitivity study; third, exactly the same samples can be used for compression simulations in quasi-static, transitional dynamic and shock regimes, thereby avoiding the widely observed experimental data scatters due to meso-scale structural difference between nominal "same" samples used in quasi-static and dynamic tests.

In early studies, mass-spring models were prevalent [222-224], which use lump masses and nonlinear springs to represent a cellular material. One-dimensional cellular chain (Fig. 28a) can be created with convenient formulation of governing equations. The lump mass and the spring rigidity normally correlate with the apparent density and the nominal quasi-static 
stress-strain relation of the cellular material, respectively. Shim et al. [224] gave the following normalised compressive force-deformation equations for the structural stiffness of the energy-dissipating "springs"

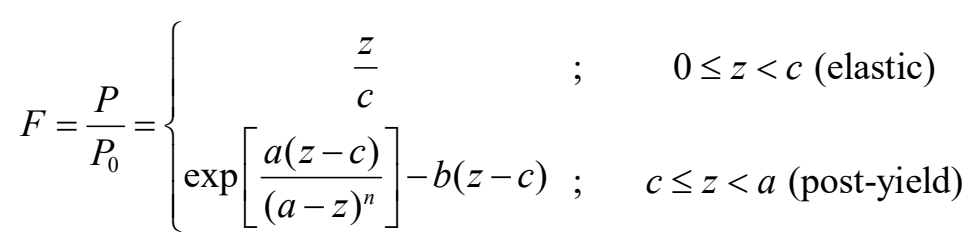

where $z$ is a variable to indicate the deformation, $a, b, c$ and $n$ are spring parameters. The governing equations for a cellular chain (Fig. 28a) are as follows

$$
\begin{aligned}
& \frac{\mathrm{d}^{2} u_{1}}{\mathrm{~d} t^{2}}+\beta_{1} F\left(z_{1}\right)=0 \text { when } i=1 \\
& \gamma_{i} \frac{\mathrm{d}^{2} u_{i}}{\mathrm{~d} t^{2}}+\beta_{i} F\left(z_{i}\right)-\beta_{i-1} F\left(z_{i-1}\right)=0 \text { when } i=2,3, \ldots, \mathrm{N}
\end{aligned}
$$

where $u_{i}=x_{i}-(i-1)$ and $z_{i}=u_{i}-u_{i+1} ; \gamma_{i}$ is the ratio of each mass in the chain to the colliding mass $\left(\mathrm{M}=\mathrm{m}_{1}\right)$ and $\beta_{i}$ is a scaling coefficient for the crushing force of the $i$ th cell.

Two-dimensional cellular array extends 1D mass-spring system by adding cross-bridges to simulate the transverse interaction between cells [225, 226] (Fig. 28b). The rigidness of cross-bridges depends on the shear rigidity of the corresponding regions and can vary from zero to infinite. Different types of dynamic loading can be applied and the structural response can be predicted.
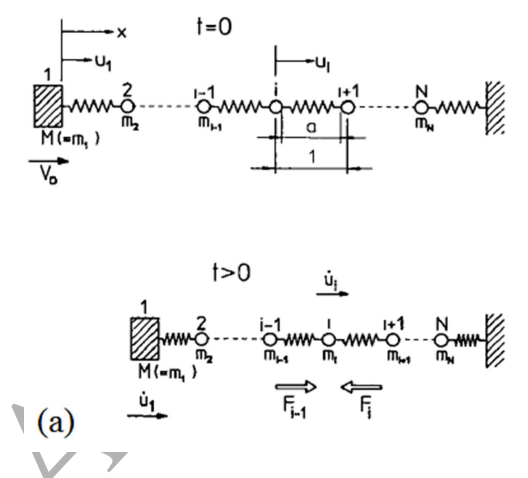

(b)

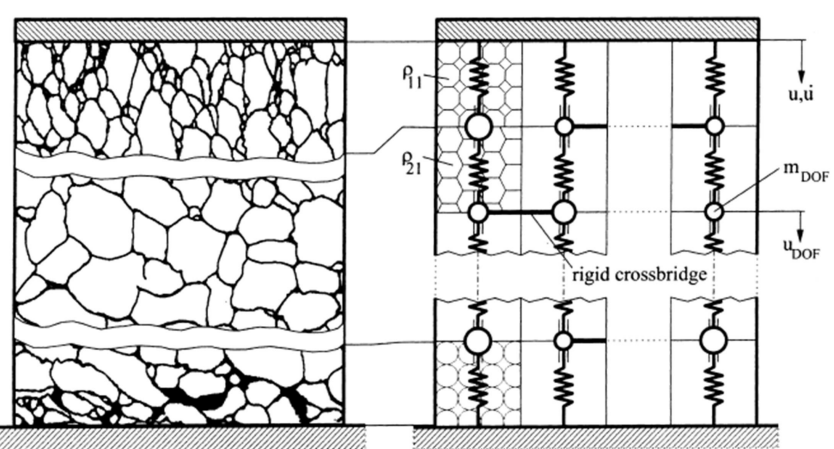

Fig. 28. Mass-spring models to predict dynamic load transmission in cellular materials: (a) 1D cellular chain [224]; (b) 2D array of lump masses, spring elements and cross bridges, to represent the cross-section along loading direction [226].

Based on mass-spring models, Shim et al. [224] studied the effects of inhomogeneity, cell damage and strain-rate on the dynamic deformation of a cellular material with consideration 
of strain softening of rigidity. Li and Meng [73] studied the shock load transmission in a cellular material subjected to a rectangular pressure pulse. Daxner et al. [226] investigated the effect of inhomogeneous apparent density on the maximum stress and energy absorption during impact.

Although mass-spring models can incorporate some meso-scale factors such as the inhomogeneity of density and rigidity, as well as strain-softening behaviour, the prediction accuracy of these models is uncertain as few of them have been validated by experimental results. Harrigan et al. [171] compared the predictions of the continuum-based shock model and mass-spring model for a cellular material subjected to a rectangular pulse. They found significant discrepancy between the predictions when the pressure is larger than twice the plateau stress. They argued that mass-spring models cannot account for the extra energy dissipation during shock wave propagation, because mass-spring models assume that each cell (or each pair of mass and spring) follows the same loading path as the predefined "stress-strain relation" of the spring, while in continuum-based shock model (Section 4.2.1) the macroscopic stress-strain state jumps after shock front passes (i.e. Rayleigh chord). As continuum-based shock model has been validated by experimental results (Section 4.2.1), they suggested that mass-spring models should be used with caution to analyse shock problems.

\subsubsection{Cell-based models}

For each cell in a cellular material, the base material and the internal gas can be treated as two distinctive "materials" of which the latter is usually assumed to have zero rigidity and strength, except when the gas is trapped [34, 212-214, 220]. An analytical model based on a cubic unit cell was developed by Gibson and Ashby [1] to derive a series of scaling laws, which have been widely used to correlate the macroscopic properties (e.g. quasi-static elastic modulus and yield strength) with the relative density and base material properties of cellular materials. However, this kind of analytical model is normally limited to small and uniform deformation, which is inadequate for dynamic compression of cellular materials for which more localised large deformation and more complicated structural factors are involved. The classical unit cell model oversimplifies the cell geometry which may play important roles in the macroscopic material properties [28-33, 36, 37, 40, 41, 227, 228]. For honeycombs with regularly-shaped, periodically-arrayed cells, the theory of structural dynamics can be applied in analytical models to predict the dynamic compressive response. For instance, Hu et al. [163, 229-231] employed a representative block of cells exhibiting a known deformation mode to 
deduce the crushing stress of different types of honeycombs subjected to impact with different loading speeds, and their analytical predictions agreed well with numerical simulations.

Theoretical analyses using cell-based models normally require that, (1) the deformation mode is known beforehand; (2) the deformation is uniform or repeatable; (3) the cells are regular and periodic. However, such requirements cannot be fully satisfied in stochastic cellular materials such as foams, and consequently these requirements limit the application of the cell-based analytical models. Many cell-based numerical models have been thus developed to overcome the difficulty faced by analytical models, and the finite element modelling of quasi-static behaviour of cellular materials has been reviewed in Ref. [232]. This section focuses on the cell-based models to investigate the dynamic compressive behaviour of cellular materials. In the models considered here, the dynamic loading is usually imposed by the constant loading rate on the sample ends or the contact between the sample and the loading platens subjected to certain boundary conditions (e.g. blast pressure and mass impact). Typical loading scenarios are illustrated in Table 1 and Table 3.

With the advances of powerful computers and software, meso-scale cell-based models have incorporated more realistic cell structures. Two types of cell-based models have been widely used. One is based on idealised cell structures, such as Kelvin, Voronoi and regularly shaped/patterned cells, as shown in Fig. 29(a)-(e) and Fig. 30(a) and (b), which are mainly used to gain general insights or represent some cellular materials by average parameters (e.g. relative density and cell size). The effects of cell structure can be examined via varying some parameters that control the formation of the morphology and topology (e.g. Fig. 29e) of the idealised cells. The other type, so-called image-based modelling [189], incorporates actual cell geometry obtained from CT images (or other imaging techniques), see Fig. 29(f) and Fig. 30(c)-(e), thereby capturing the actual meso-scale structural effect for a specific cellular material imaged. The recent advances of the hardware for imaging (e.g. industrial CT and high performance computer) and numerical codes (e.g. image processing and FE meshing) have led to the increasing applications of image-based modelling to cellular materials [189, $233,234]$. It is also possible to generate idealised cell structures using structural information extracted from CT images. For instance, the actual distributions of cell size and cell-wall thickness, and the number of edges or faces per cell can be used as input parameters when generating an idealised cell-based FE model $[158,234,235]$. In this way, the FE model can use structural elements (e.g. beam and shell) to mesh the virtual sample with similar structural statistics to a real cellular material, which avoids the use of continuum elements (necessary to represent complex geometry) which are susceptible to distortion and are less computationally 
efficient. It should be also mentioned that some mesh-free methods (e.g. material point method $[236,237])$ have been proposed in image-based modelling to circumvent meshing difficulty and to handle extreme deformation and severe contact problems.

The image-based FE modelling has been employed to investigate the compressive properties of a variety of cellular materials such as open-cell aluminium sponge/foam [190, 238], closed-cell aluminium foam [24-26, 165, 238-242], steel hollow sphere structure [235] and metallic fibre structure [243]. These modelling results are valuable to explain the compressive behaviour of the imaged cellular materials. For instance, the strain-rate effect of Alporas foam and m.pore ${ }^{\mathrm{TM}}$ foam have been investigated by 3D image-based modelling, see Fig. 17 and Fig. 18. The main benefit of these models is that the effect of base material properties can be reliably identified, as the actual mesoscopic celt structure has been incorporated in modelling. However, the high fidelity of cell geometry also causes the limitation of image-based modelling, i.e. the cell structure is speeific to the imaged sample and the structural parameters cannot be intentionally/changed to examine their effect. Moreover, image-based modelling requires sophisticated (usually expensive) imaging and computing facilities to construct the model and implement the computation. This usually restricts the sample size and loading cases that can be considered $[25,26]$. Therefore, most of the current cell-based models use idealised cell structures. In particular, extensive dynamic simulations of 2D cellular materials have been performed (Fig. 29).

(a)

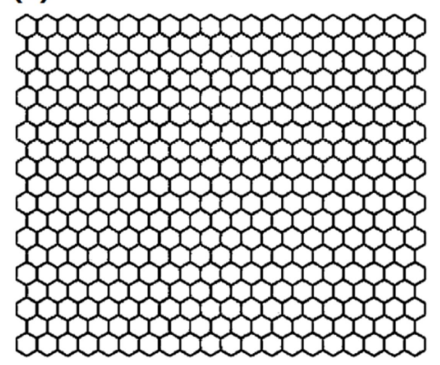

(d)

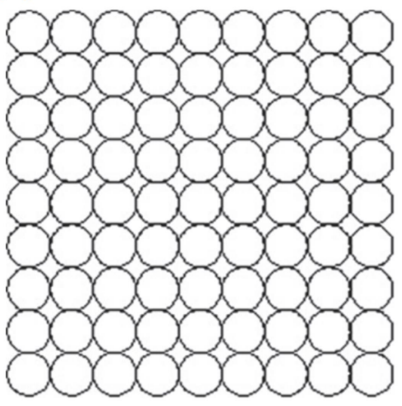

(b)

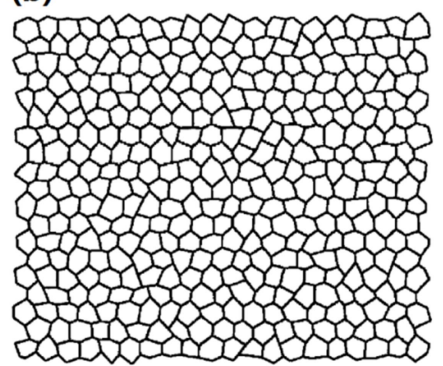

(e)

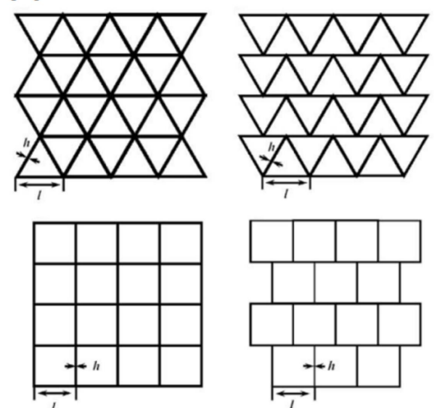

(c)

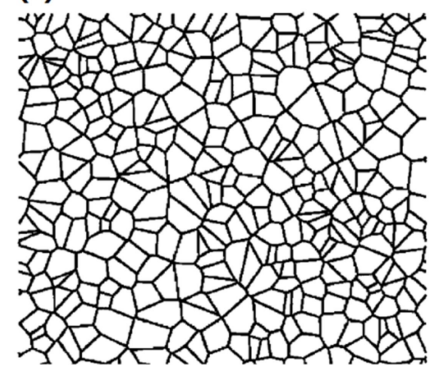

(f)

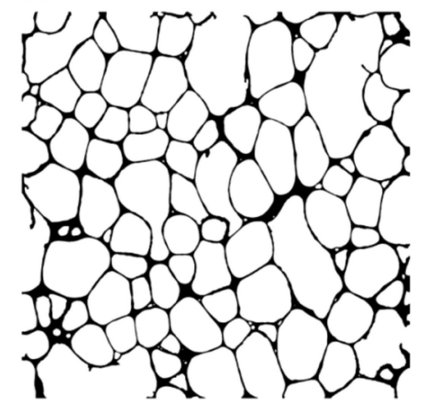

Fig. 29. Typical 2D cell structures used in numerical simulations, which consist of: (a) regular 
hexagonal cells [67]; (b) disordered hexagonal cells [67]; (c) Voronoi cells [67]; (d) circular cells [244]; (e) triangular and rectangular cells with regular and staggered arrangements [38]; (f) CT-image-based cells [25].

Regular hexagonal-cell structures were used early to analyse the deformation mechanisms, as well as their effect on compressive stress. For instance, Honig and Stronge [174, 175] demonstrated the elastic wave propagation and the trapping of plastic deformation in the in-plane compression of honeycomb using FE model. Ruan et al. [66] simulated the in-plane dynamic crushing of honeycombs at various impact velocities and observed so-called " $\mathrm{X}$ ", "V" and "I" deformation modes with increasing impact velocity for the $\mathrm{X}_{1}$-direction crushing, while only "V" and "I" deformation modes for the $\mathrm{X}_{2}$-direction crushing. These simulations for regular cell structures were also compared with experimental results of aluminium honeycombs. Subsequently, randomly distributed cells were introduced. For instance, Zheng et al. [67] generated hexagonal, disordered and Voronoi cell structures, respectively, and performed crushing simulations for different cell irregularities and impact velocities. They identified three deformation modes for 2D cellular materials, i.e. quasi-static one with localised collapse bands, transitional one with both shear and transverse bands, and dynamic one with layerwise collapse bands, and these deformation modes appear sequentially with increasing the impact velocity.

Three-dimensional simulations appeared later, but they have been increasingly used recently (Fig. 30). For instance, Song et al. [245] constructed 3D Kelvin and Voronoi cell structures, and performed dynamic crushing simulations at various loading speeds. They found that the plateau stress and energy absorption can be enhanced by increasing geometric irregularity, impact velocity and strain hardening. Zheng et al. [149] used a 3D Voronoi foam to establish the dynamic constitutive relation for shock stress-strain states and furthermore to inform experimental measurement of this relation. 
(a)

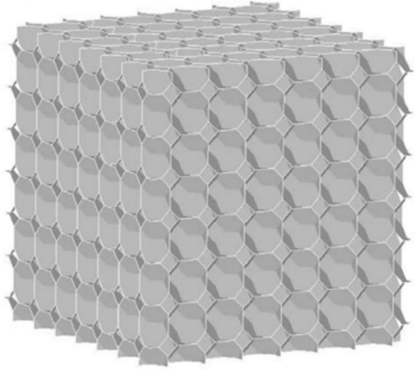

(b)

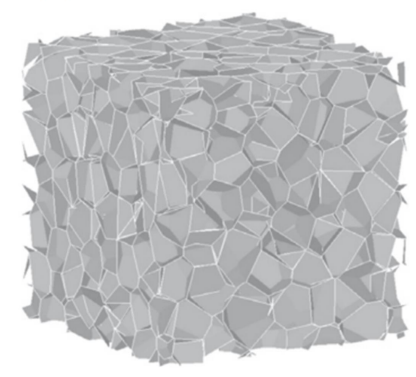

(d)

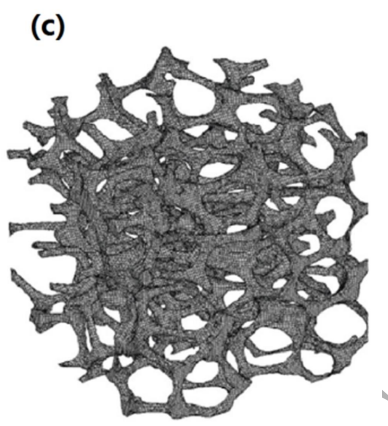

(e)

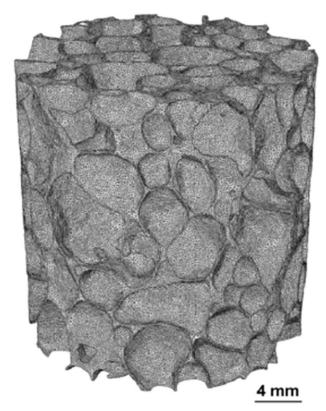

Fig. 30. Typical 3D cell structures used in numerical simulations, which consist of: (a) tetrakaidecahedron (or Kelvin) closed cells [245]; (b) Voronoi closed cells [245]; (c) CT-image-based open cells [190]; (d) CT-image-based closed cells (a small sample with cross-section shown) [26]; (e) CT-image-based closéd cells (a large sample) [26].

In general, the $2 \mathrm{D}$ models predict compressive behaviour of cellular materials in agreement, at least qualitatiyely, with most of the $3 \mathrm{D}$ modelling results and experimental observations. Due to their reduced computational cost, large samples and extensive loading cases can be considered, which have contributed significantly to our understanding of the dynamic compressive behaviour of cellular materials. Taking shock compression as an example, Fig. 31 shows an FE model for a sufficiently long hexagonal-cell honeycomb sample, as well as the predicted distributions of local velocity and stress using a cross-seetional nodal variable averaging approach [20]. It is clearly seen that both material velocity and stress jump across a distance of the order of one cell size, demonstrating the features of shock front, which is much more difficult to quantify in experiments. Another local strain measure was proposed and applied to 2D Voronoi cell structures [22, 23], as shown in Fig. 32. This approach tracks the motion of each FE node and calculates the local strain at a macroscopic material point using the nodal distances and their change within a circular neighbourhood. Fig. 32b and Fig. 32c show the calculated local strain field, which is consistent with experimental DIC local strain measurements for foams, see Fig. 9. 
Furthermore, the location of the maximum local strain gradient across the shock front, which has a mesoscopic finite thickness, can be used to define the location of the shock front of zero thickness at the macro-scale.
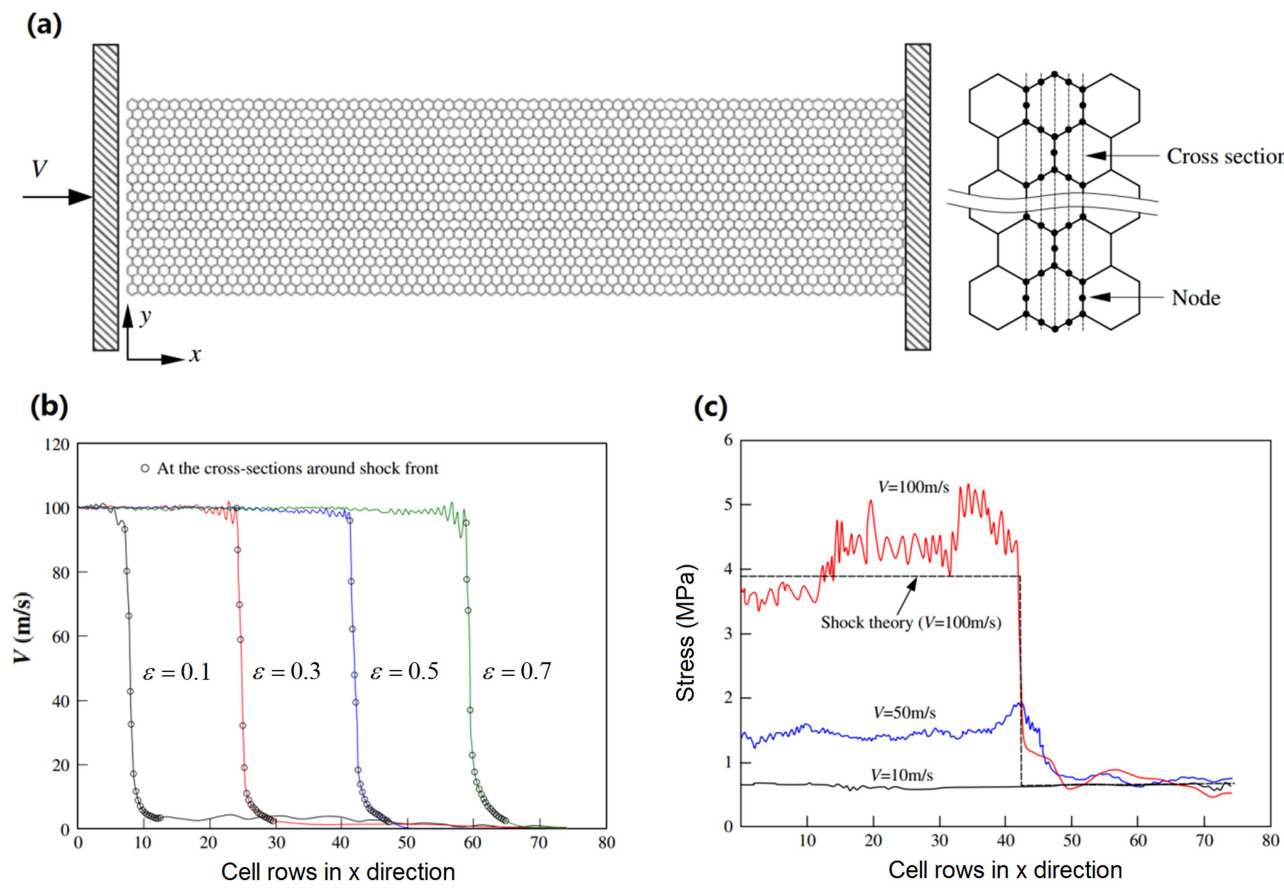

Fig. 31. Numerical simulations to demonstrate shock front features in honeycomb [20]: (a) honeycomb specimen and its cross-sections for definitions of local quantities; (b) distribution of the sectional velocity at an impact velocity of $100 \mathrm{~m} / \mathrm{s}$; (c) distribution of compressive stress in the honeycomb at different loading speeds. 


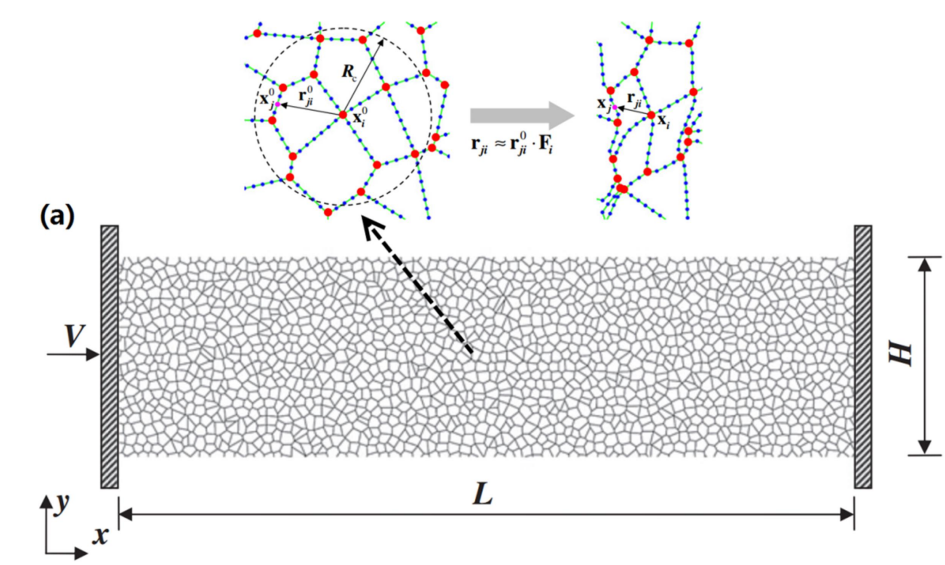

(b)
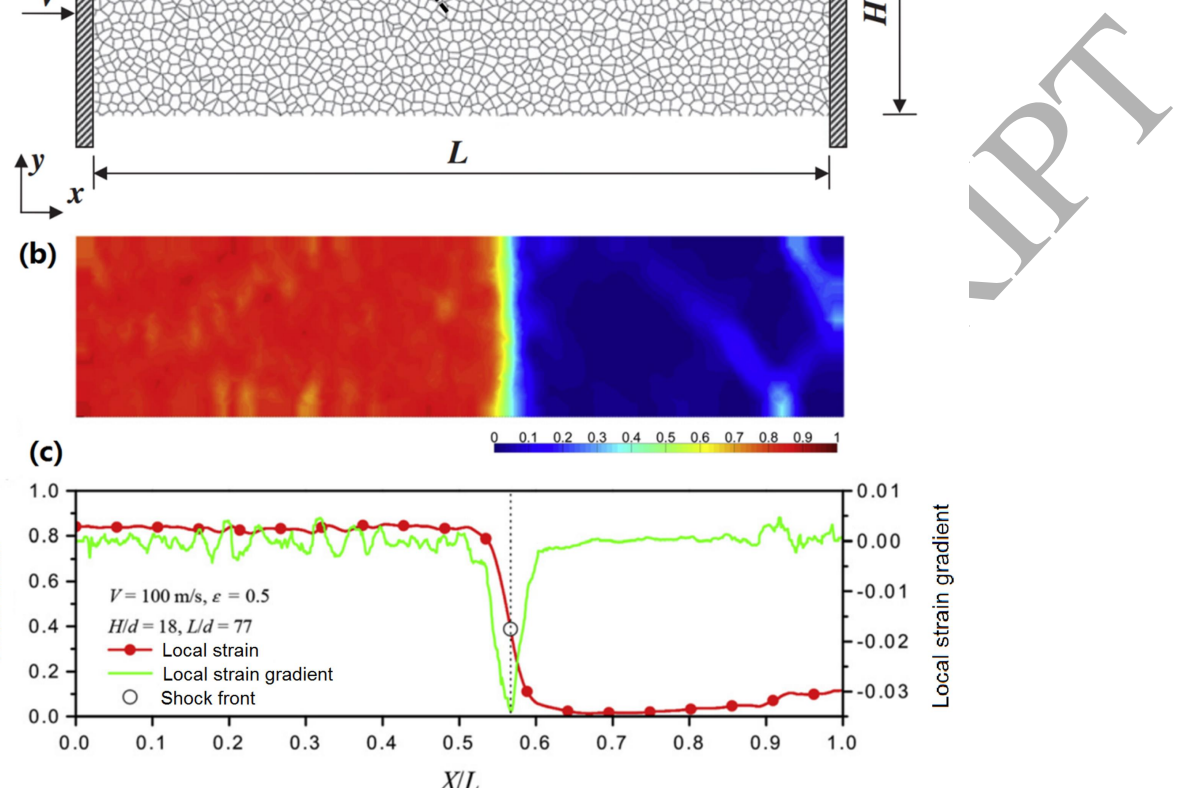

$X / L$

Fig. 32. Numerical simulations to determine shock strain in 2D Voronoi foam (adapted from Ref. [22]): (a) finite element model and schematic of nodal motion in the neighbourhood of a corner node $i$ with position vectors $\boldsymbol{x}_{i}^{0}$ and $\boldsymbol{x}_{i}$ in the reference and current configurations, respectively; (b) colour map of local strain field for macroscopic material points and (c) the corresponding distributions of local strain and local strain gradient.

The cell-based models are particularly useful to quantitatively examine the effects of mesoscopic structural parameters and homogenised base material properties on the dynamic compressive behaviour of cellular materials. Extensive numerical studies [34, 42-44, 59, 67, $70,105,157,159,228,245]$ have been devoted to revealing the roles of cell shape, cell-wall thickness, cell size, structural defects, relative density and base material properties (e.g. Young's modulus, Poisson's ratio, strain hardening and strain-rate hardening) in controlling dynamic compressive properties. The structural imperfections (e.g. heterogeneities in cell shape and cell-wall thickness) cause deformation modes with more randomness in quasi-static and transitional dynamic regimes, but they do not change the deformation mode in shock regime (i.e. propagation of planar wave front) [43, 67, 245]. For Voronoi foams, some numerical studies showed that the plateau stress $[67,245]$ and the energy absorption $[42,245]$ 
increased with increasing the irregularity of cell shape, but Li et al. [43] found that both the irregularity of cell shape and the nonuniform thickness of cell walls led to decreases in plateau stress and strain energy. These conflicting conclusions about the effect of cell shape irregularity may be explained by another numerical study [105] which indicates that plateau stress decreases with increasing the degree of cell shape irregularity varying from 0.2 to larger values, but when the irregularity value is smaller than 0.2 the shape irregularity enhances the plateau stress instead. The structural defects generally reduce the plateau stress and energy dissipation capacity $[42,44]$. The strain hardening can significantly increase the dynamic crushing stress [245]. When relative density increases, the dynamic plateau stress, energy absorption and shock speed all increase, but the role of the relative density in dynamic compression is quite different from that in quasi-static compression [1, 43, 157, 159, 245]. The strain-rate hardening increases the dynamic crushing stress $[59,70]$ and it has significant effect on the shock speed and shock stress [157]. Interestingly, the eell size has a considerable effect on dynamic crushing stress only when it is combined with base material strain-rate hardening [228]. Consistent findings have been reported with regard to the effects of relative density, strain hardening and strain-rate hardening, but the mechanisms for other influential factors need further verification from modelling results for different types of cell structures.

\subsection{Continuum mechanics models}

\subsubsection{One-dimensional models}

The dynamic response of a cellular material to an impact or blast load can be modelled in the framework of continuum mechanics, as long as the deformation, motion and force experienced by the cells can be treated in a continuum sense, i.e. each cell (or each basic constituent unit) of the cellular material can be represented by a "material point" from a macroscopie point of view. In general, continuum mechanics models are more efficient and versatile, compared to the cell-based models (Section 4.1). They comply with the fundamental principles (e.g. conservation laws) and are generally consistent with the micro- and meso-scale mechanisms discussed in Section 3. The first task for a continuum-based model is the establishment of a constitutive relation, which is usually a stress-strain relation but also can be other ones [25, 69]. A number of simplified stress-strain relations have been proposed to model the 1D compressive constitutive behaviour of cellular materials, and the most widely used ones are shown in Fig. 33. The initial compressive response before the plateau stage is usually assumed linearly elastic, which is acceptable if large deformation is of major interest. 
Accordingly, the yield stress $\sigma_{\mathrm{y}}$ (normally adopted as collapse initiation stress $\sigma_{\mathrm{c} 0}$, see Fig. 5) and the yield strain $\varepsilon_{y}$ (normally adopted as collapse initiation strain $\varepsilon_{\mathrm{c} 0}$, see Fig. 5) indicate the beginning of the plastic response. The plasticity in the plateau stage can be assumed perfect, linear hardening or non-linear hardening. In the early shock studies [e.g. 153], the plateau stage and the densification stage were usually separated; and a densification initiation strain, namely $\varepsilon_{\mathrm{d} 0}$, thus needs to be defined to indicate the onset of densification [100], see Fig. 5.

The widely used simplifications of the stress-strain relations include "elastic, perfectly plastic, rigid" (E-PP-R), "elastic, linear hardening plastic, rigid" (E-LHP-R), "elastic, perfectly plastic, non-linear hardening" (E-PP-NLH), "elastic, non-linear hardening plastic" (E-NLHP) and "elastic, softening, hardening" (E-S-H) simplifications, as shown in Fig. 33. A rigid assumption in the densification stage is also called lock-up or locking by some researchers (e.g. [73, 153]); in other words, the material is locked or frozen to the densification initiation strain $[54,55,153]$ (or the maximum strain in the densification stage [148]). Hence the ' $R$ ' in E-PP-R and E-LHP-R is often replaced by ' $L$ '. When the initial elastic part is neglected, a rigid plasticity is assumed, and then the ' $\mathrm{E}$ ' in E-PP-R, E-LHP-R, E-PP-NLH, E-NLHP and E-S-H should be replaced by 'R'. All of these abbreviations refer to the simplification of the quasi-static stress-strain relation, unless otherwise stated.

The linear hardening plasticity can be simply described by a hardening modulus, namely $E_{\mathrm{p}}$, whereas for nonlinear hardening plasticity, different functions have been proposed to fit experimental data. For instance, Hanssen et al. [246] proposed a complicated nonlinear function as follows

$$
\sigma=\sigma_{\mathrm{y}}+\chi \frac{\varepsilon}{1-\rho / \rho_{\mathrm{s}}}+\alpha \ln \left[1-\left(\frac{\varepsilon}{1-\rho / \rho_{\mathrm{s}}}\right)^{\beta}\right]^{-1}
$$

where $x, \alpha$ and $\beta$ are material constants. Pattofatto et al. [210] suggested the below power function

$$
\sigma=\sigma_{\mathrm{y}}+K \varepsilon^{n}
$$

where $K$ and $n$ are hardening constants. Despite the simplicity, Eq. (24) gives a maximum stress value, namely $\sigma_{\mathrm{y}}+K$, when approaching compression limit $(\varepsilon=1)$. By contrast, the stress predicted from Eq. (23) will be infinite when the strain is equal to $1-\rho / \rho_{s}$ which may be regarded as the full densification of the cellular material if lateral deformation is negligible, and thus Eq. (23) is usually more capable of fitting test data at very large 
deformation (accordingly, very high stress) [246]. To meet both the simplicity and accuracy requirements, the below function can be used

$$
\sigma=\sigma_{\mathrm{y}}+A \varepsilon(1-B \varepsilon)^{-n}
$$

which is a generalised form of Eqs. (10) and (11). When $n=2$, this equation can be reduced to the stress-strain Hugoniot relation for shock, as derived from a linear Hugoniot relation between shock speed and impact velocity [25, 69]. Zheng et al. [149] adopted that $n=2$ and $B=1$, and they obtained good fit to their numerical simulation results for both quasi-static and dynamic compressions of a 3D Voronoi foam.

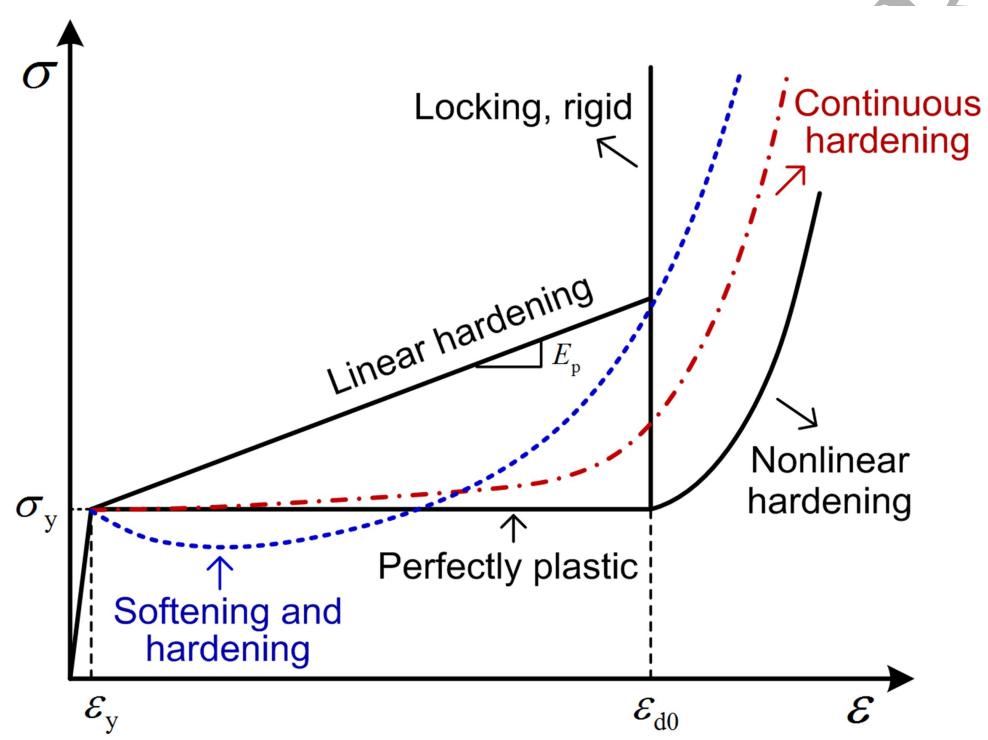

Fig. 33. Simplified quasi-static compressive stress-strain relations of cellular materials.

If the stress plateau is flat but a rigid assumption is not acceptable in the densification stage, an E-PP-NLH or R-PP-NLH simplification may be more appropriate. For instance, Li and Meng [73] used below function to describe the strain hardening in the densification stage

$$
\sigma=\sigma_{\mathrm{y}}+\delta \sigma_{\mathrm{y}}\left\{\tan \left[\frac{\pi}{2}\left((1-\mu)+\mu \frac{\varepsilon-\varepsilon_{\mathrm{d} 0}}{1-\varepsilon_{\mathrm{d} 0}}\right)\right]-\tan \left[\frac{\pi}{2}(1-\mu)\right]\right\}
$$

where $\delta$ and $\mu$ are material parameters. However, as demonstrated by Harrigan et al. [171], this complicated function is not convenient for analytical solution.

When softening in the plateau stage is considered, a non-monotonic function can be used. For instance, Harrigan et al. [154] proposed the following stress-strain equation for American oak compressed along grain 


$$
\sigma=\sigma_{\mathrm{y}}\left(1-A \varepsilon \frac{\varepsilon_{1}-\varepsilon}{\varepsilon_{2}-\varepsilon}\right)
$$

where $A$ is a material parameter, and $\varepsilon_{1}$ and $\varepsilon_{2}$ are two reference strains controlling the softening and hardening, with $\varepsilon_{1}<\varepsilon_{2}$. A combination of softening and hardening was also considered by Shim et al. [224] using a different simplification, see Eq. (20).

Several models have been proposed to take account of the strain-rate hardening. For instance, Gibson and Ashby [1] suggested the below equation for the dynamic plastic collapse stress of metallic cellular materials

$$
\frac{\sigma_{\mathrm{y}}^{\mathrm{d}}}{\sigma_{\mathrm{y} 0}}=1-A \frac{T}{T_{\mathrm{m}}} \ln \frac{\dot{\varepsilon}^{*}}{\dot{\varepsilon}}
$$

where $\sigma_{\mathrm{y} 0}$ is the plastic collapse stress at $0 \mathrm{~K} ; T$ and $T_{\mathrm{m}}$ are current temperature and melting temperature of the base material, respectively; $A$ and $\dot{\varepsilon}^{*}$ are material constants.

Zhang et al. [122] were motivated by Nagy et al. [121] and proposed a power law with an exponent being a function of strain to predict the dynamic yield stress, viz.

$$
\frac{\sigma(\varepsilon)}{\sigma_{0}(\varepsilon)}=\left(\frac{\dot{\varepsilon}}{\dot{\varepsilon}^{*}}\right)^{a+b \varepsilon}
$$

where $\sigma_{0}(\varepsilon)$ represents the quasi-static stress-strain relation of a cellular material at a reference strain-rate $\dot{\varepsilon}^{*}$, and $a$ and $b$ are material constants. This model distinguishes the strain-rate hardening at different strains.

Ruan et al. [247] developed another empirical formula to fit the experimentally measured plateau stress, viz.

$$
\frac{\sigma_{\mathrm{pl}}}{\sigma_{\mathrm{ys}}}=A\left(1+B \dot{\varepsilon}^{m}\right)\left(\frac{\rho}{\rho_{s}}\right)^{n}
$$

where $\rho / \rho_{s}$ is relative density, $\sigma_{y s}$ is yield strength of the base material, and $A, B, m$ and $n$ are material constants. For Alporas foam, it is reported that $A=0.59, B=0.14, m=0.17$ and $n=1,7 \quad$ [107].

Other empirical rate-dependence equations for traditional engineering materials may be also used in the modelling of cellular materials. For instance, the Cowper-Symonds equation describes the rate dependence of yield stress, which is expressed as

$$
\frac{\sigma_{\mathrm{y}}^{\mathrm{d}}}{\sigma_{\mathrm{y}}^{\mathrm{s}}}=1+\left(\frac{\dot{\varepsilon}}{\dot{\varepsilon}^{*}}\right)^{\frac{1}{m}}
$$

where $\sigma_{\mathrm{y}}^{\mathrm{d}}$ and $\sigma_{\mathrm{y}}^{\mathrm{s}}$ are the dynamic and static yield stresses, respectively; $m$ is an empirical 
exponent; and $\dot{\varepsilon}^{*}$ is a reference strain-rate at which the dynamic yield stress is twice the static one. In commercial FE software Abaqus, the strain-rate effect in the crushable foam model can be incorporated by using Cowper-Symonds equation [248].

The above rate-dependence equations for dynamic loading can be used for the strain-rate effect on plastic properties in transitional dynamic regime. However, they imply that the stress-strain relation varies with strain-rate and the rate-dependence is taken into account by modifying the quasi-static stress-strain relation. Such a treatment may be inappropriate for shock compression of cellular materials, which has been shown to conform to a unique dynamic stress-strain relation for different loading speeds [25, 69, 149, 158]. A different treatment is thus needed for shock regime. For instance, Zheng et al. [149] adopted a special form of Eq. (25), i.e. $B=1$ and $n=2$, to describe the constitutive stress-strain relation of a 3D Voronoi foam in both quasi-static and shock compressions. However, different material parameters were used, i.e. $\sigma_{\mathrm{y}}=5.9 \mathrm{MPa}$ and $A=0.6 \mathrm{MPa}$ for quasi-static compression, while $\sigma_{\mathrm{y}}=7.7 \mathrm{MPa}$ and $A=0.22 \mathrm{MPa}$ for shock compression. Note that neither strain-rate nor loading speed was included in Eq. (25), as a treatment distinct from Eqs. (28)-(31).

The fundamental conservation laws are another essential aspect of continuum mechanics model. The equations for conservations of mass, momentum and energy can be used to establish governing equations for a dynamic problem. Herein the engineering strain $\varepsilon$ and stress $\sigma$ are defined as positive in compression. When shock is absent, the below wave equation (neglecting body force) can be used to obtain the particle motion during uniaxial compression

$$
\frac{\partial^{2} u}{\partial t^{2}}-C^{2} \frac{\partial^{2} u}{\partial X^{2}}=0
$$

where $u$ is displacement, $t$ is time, $X$ is Lagrangian location, and $C=\sqrt{(d \sigma / d \varepsilon) / \rho_{0}}$ obtained from constitutive stress-strain relation.

For a shock wave, differential equations are not applicable due to the discontinuity, and thus jump equations are needed. According to $1 \mathrm{D}$ shock wave theory $[55,150]$, the physical quantities across the shock front must satisfy the conditions associated with the singular surface, i.e. the Rankine-Hugoniot relations. Here we use symbols "s", "a" and "b" to denote "shock front", "ahead of" and "behind" the shock front, respectively, and the equations are given with respect to the Lagrangian reference configuration, see Fig. 34. The kinematic compatibility reflecting the mass conservation leads to the below relationship between velocity jump and strain jump across the shock front 


$$
V_{\mathrm{b}}-V_{\mathrm{a}}=V_{\mathrm{s}}\left(\varepsilon_{\mathrm{b}}-\varepsilon_{\mathrm{a}}\right)
$$

where $V_{\mathrm{s}}$ is the Lagrangian velocity of the shock front (i.e. the velocity of the shock front irrespective to material motion). The absolute velocity of shock front can be expressed as

$$
v_{\mathrm{s}}=\frac{V_{\mathrm{b}}-V_{\mathrm{a}}}{\varepsilon_{\mathrm{b}}-\varepsilon_{\mathrm{a}}}\left(1-\varepsilon_{\mathrm{a}}\right)+V_{\mathrm{a}}=\frac{V_{\mathrm{b}}-V_{\mathrm{a}}}{\varepsilon_{\mathrm{b}}-\varepsilon_{\mathrm{a}}}\left(1-\varepsilon_{\mathrm{b}}\right)+V_{\mathrm{b}}
$$

which is equal to $V_{\mathrm{s}}$ when $V_{\mathrm{a}}=0 \mathrm{~m} / \mathrm{s}$ and $\varepsilon_{\mathrm{a}}=0$.

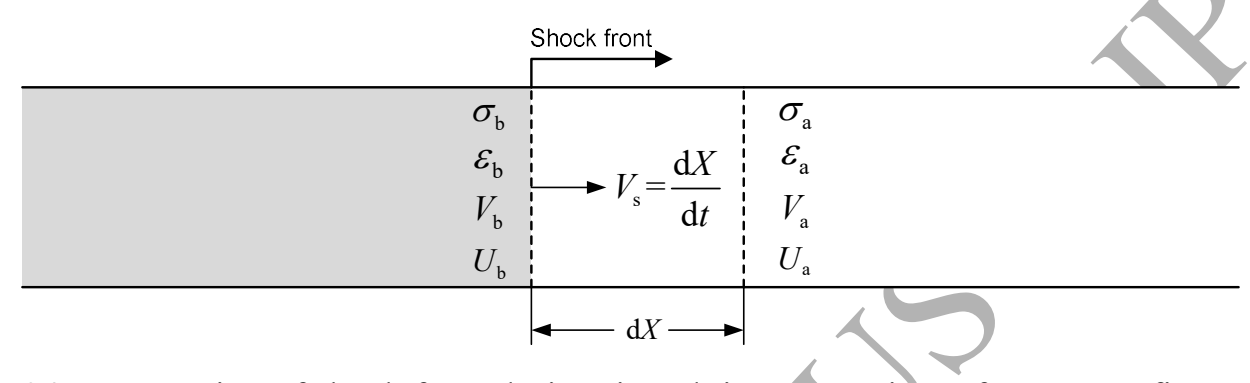

Fig. 34. Propagation of shock front during time $\mathrm{d} t$ in Lagrangian reference configuration.

The dynamic compatibility reflecting the momentum conservation leads to the following relationship between the stress jump and velocity jump

$$
\sigma_{\mathrm{b}}-\sigma_{\mathrm{a}}=\rho_{0} V_{\mathrm{s}}\left(V_{\mathrm{b}}-V_{\mathrm{a}}\right)
$$

where $\rho_{0}$ is the initial material density. Substituting Eq. (33) into Eq. (35), we can obtain the shock speed as follows

$$
V_{\mathrm{s}}=\sqrt{\frac{\sigma_{\mathrm{b}}-\sigma_{\mathrm{a}}}{\rho_{0}\left(\varepsilon_{\mathrm{b}}-\varepsilon_{\mathrm{a}}\right)}}
$$

In the stress-strain plane, Rayleigh line with a slope equal to $\rho_{0} V_{\mathrm{s}}^{2}$ connects the initial state (yielding state if elastic precursor exists) and the final state when a shock wave passes. Indeed, Ding et al. [156] has numerically demonstrated that the stress-strain states of the mesoscopic material points of a 3D Voronoi foam follow the trajectory defined by the Rayleigh line when a shock wave passes these mesoscopic material points.

Eliminating $V_{\mathrm{s}}$ in Eqs. (33) and (35), we can further obtain

$$
\sigma_{\mathrm{b}}-\sigma_{\mathrm{a}}=\rho_{0} \frac{\left(V_{\mathrm{b}}-V_{\mathrm{a}}\right)^{2}}{\varepsilon_{\mathrm{b}}-\varepsilon_{\mathrm{a}}}
$$

which gives the stress jump across the shock front. And then the energy conservation in an adiabatic process leads to 


$$
U_{\mathrm{b}}-U_{\mathrm{a}}=\frac{\left(\sigma_{\mathrm{b}}+\sigma_{\mathrm{a}}\right)\left(\varepsilon_{\mathrm{b}}-\varepsilon_{\mathrm{a}}\right)}{2}
$$

where $U$ is the internal energy density. Note that the derivations of Eqs. (33)-(38) do not involve any constitutive constants and they are valid in any continuum solid. A more general form of Eq. (38) can be written as follows to account for heat flow [55]

$$
U_{\mathrm{b}}-U_{\mathrm{a}}=\frac{\left(\sigma_{\mathrm{b}}+\sigma_{\mathrm{a}}\right)\left(\varepsilon_{\mathrm{b}}-\varepsilon_{\mathrm{a}}\right)}{2}+\frac{q_{\mathrm{b}}-q_{\mathrm{a}}}{V_{\mathrm{s}}}
$$

where $q$ is heat flux density. According to the second law of thermodynamics, we have

$$
\theta_{\mathrm{b}}-\theta_{\mathrm{a}} \geq \frac{(q / T)_{\mathrm{b}}-(q / T)_{\mathrm{a}}}{V_{\mathrm{s}}}
$$

where $\theta$ is the entropy density and $T$ is the absolute temperature. Assuming an adiabatic condition, Darvizeh and Davey [249] estimated the temperature rise during shock propagation and they found that the maximum temperature rise is below $50 \mathrm{~K}$ for typical parameters adopted for an aluminium cellular bar.

It is interesting to note that the internal energy dissipated after a shock wave passes is determined by the area under Rayleigh line, which is larger than the strain energy calculated by integrating quasi-static or dynamic stress-strain curve. This is a common feature for a shock in a solid $[150,155]$. The micro- and meso-scale mechanisms of the additional energy dissipation in shock compression of cellular materials have been discussed in Sections 3.1.1 and 3.2.1. To account for the energy absorption in the macroscopic theoretical framework, Tan et al. [55] used the thermal-mechanical approach to analyse 1D shock problem and showed that the establishment of global energy balance based on the quasi-static stress-strain curve by some researchers [e.g. 250, 251] is incorrect. Harrigan et al. [171] further discussed this issue and they concluded that the application of conservation equations for mass and momentum is sufficient to obtain correct solution to a shock problem, while the "energy balance" approach fails, due to its ignorance of the extra energy dissipated during shock propagation.

Table 3 lists the typical loading scenarios considered for analytical shock models reported in the literature. Apart from the various loading scenarios considered, the analytical models adopted different constitutive stress-strain relations. As the same conservation laws of mass, momentum and energy have to be applied, a proper material model will be a decisive factor affecting the prediction accuracy.

Reid and Peng [153] first analysed the shock problem in cellular materials. They considered the direct impact response (Scenario 1, see Table 3) of wood and obtained 
analytical solution using quasi-static R-PP-L material model, and the prediction is in reasonable agreement with experimental results, as shown in Fig. 35a. However, they noticed that the prediction for impact response along the wood grain $\left(0^{\circ}\right)$ is less accurate using R-PP-L material model, mainly because in such a case the wood exhibits strain-rate hardening due to micro-inertia effect (Section 3.1.2). Subsequently, Harrigan et al. [154] improved the analytical prediction using a dynamic stress-strain relation (i.e. E-S-H and R-S-H) that takes into account the micro-inertia effect, as shown in Fig. 35a. The analytical prediction of impact response of Hydro/Cymat aluminium foam can be also improved by considering the strain-hardening in the plateau stage (R-LHP-L), as shown in Fig. 35b. Furthermore, Tan et al. (2012) showed that the consideration of an increase in locking strain with impact velocity improves the analytical prediction based on R-PP-L material model for aluminium Duocel foam. The experimental work by Elnasri et al. [210] and its companion theoretical analysis by Pattofatto et al [13] showed that the shock strain is essentially dependent on impact velocity. These studies are valuable for the evaluation of the errors introduced by assuming a locking strain in the densification stage. It should be mentioned that Scenario 2 is usually considered in conjunction with Scenario 1, while the validation of analytical model is mainly based on the results for Scenario 1 for which the crushing stress behind shock front can be measured relatively easily in experiments.

For Scenario 3, Radford et al. [147] demonstrated the prediction accuracy of shock model using R-PP-L simplification (Fig. 35c) and they considered the motion of a rigid plate impinged by an aluminium Alporas foam in their model. Lopatnikov et al. [148] considered aluminium foam projectile impacting a fixed rigid wall and solved the dynamic problem based on an E-PP-L assumption. They further considered that the foam impacting on a Hopkinson pressure bar (Table 1) may introduce more complicated stress wave interaction in comparison with the foam impacting on a fixed rigid wall. Therefore, they performed numerical simulations to further understand the stress wave propagation and address the limitation of the analytical model.

For Scenario 4, Ousji et al. [252] compared the analytical prediction of shock model based on R-PP-L material idealisation with the blast experimental results, as shown in Fig. 35d. Their study highlights the effect of blast load description (triangle pulse vs. fluid-solid-interaction pulse) on the accuracy of the shock model. Ding et al. [253] derived the analytical solution to shock problem with consideration of a triangle blast pulse and their analytical prediction agrees well with the cell-based numerical simulation. They also demonstrated that the analytical prediction based on R-NLHP is more accurate than that based 
on R-PP-L. Harrigan et al. [171] considered a cellular material subjected to a rectangular pulse and adopted both R-PP-L and E-PP-NLH material models for theoretical analysis. They also compared the predictions from the continuum shock theory with those by mass-spring model [73] and found significant discrepancy in certain loading cases, as discussed in Section 4.1.1.

Table 3 Analytical models for typical scenarios of dynamic response of cellular materials

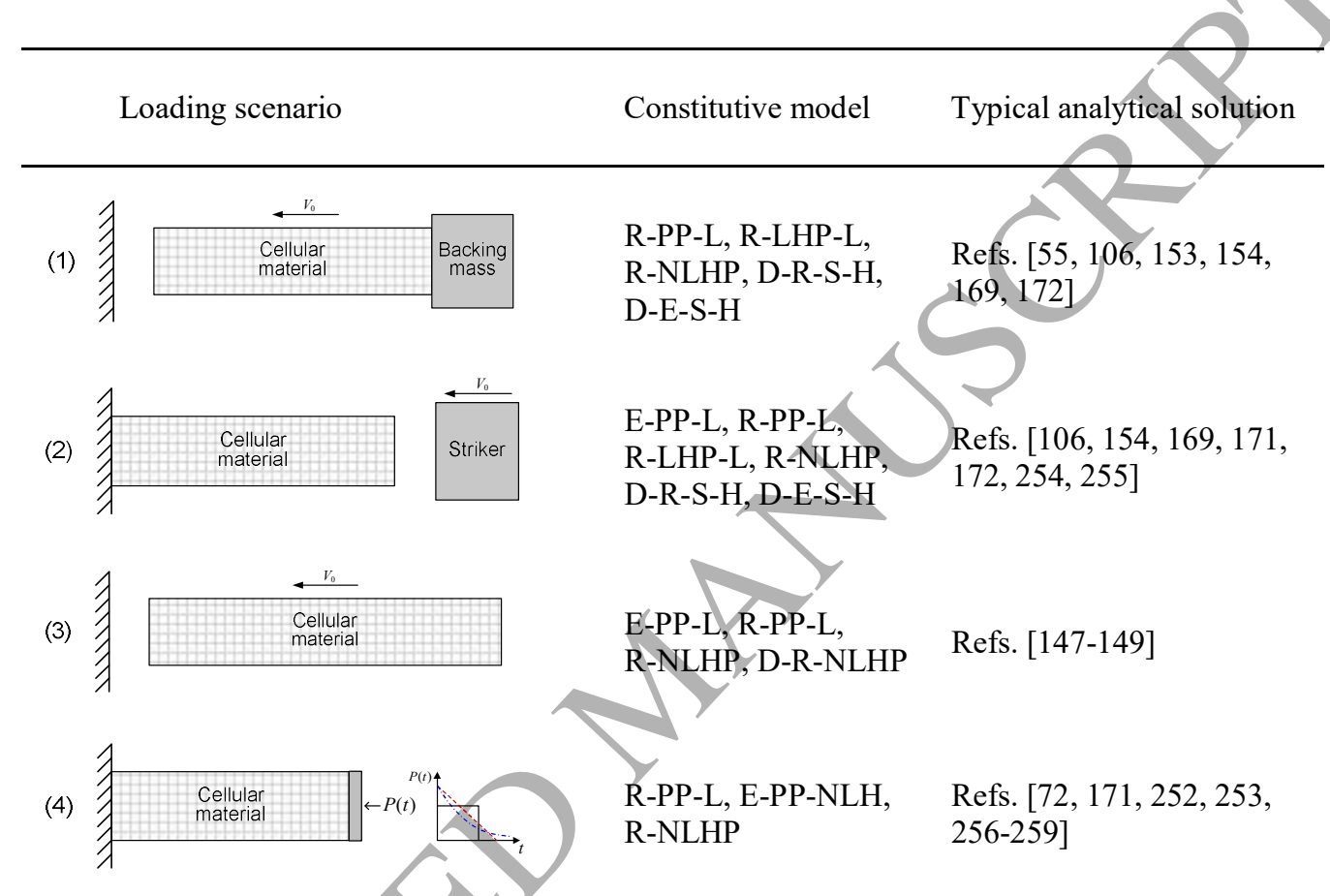

Note: (1) R-PP-L: rigid, perfectly plastic, locking (quasi-static); (2) R-LHP-L: rigid, linear hardening plastic, locking (quasi-static); (3) E-PP-L: elastic, perfectly plastic, locking (quasi-static); (4) E-PP-NLH: elastic, perfectly plastic, non-linear hardening (quasi-static); (5) R-NLHP: rigid, nonlinear hardening plastic (quasi-static); (6) D-R-NLHP: dynamic, rigid, non-linear hardening plastic; (7) D-R-S-H: dynamic, rigid, softening, hardening; (8) D-E-S-H: dynamic, elastic, softening, hardening; (9) In Scenario 4, the cover plate can be treated as rigid and of zero mass if the pressure is directly applied to the cellular material.

The above summary of typical analytical models for shock problems supports the following conclusions: (1) the simplest R-PP-L based model can capture shock behaviour in some impact and blast loading scenarios; (2) improvement can be made by considering strain-hardening in either plateau stage or densification stage, or in both plateau and densification stages (i.e. continuous hardening), thereby capturing the potential unloading and reloading effect, as well as the velocity dependence of all state variables under shock; (3) strain-rate effect must be considered to obtain accurate solution if the cellular material is 
strain-rate sensitive (e.g. wood loaded along grain); (4) the accurate description of loading or boundary condition is also important, particularly for blast response.
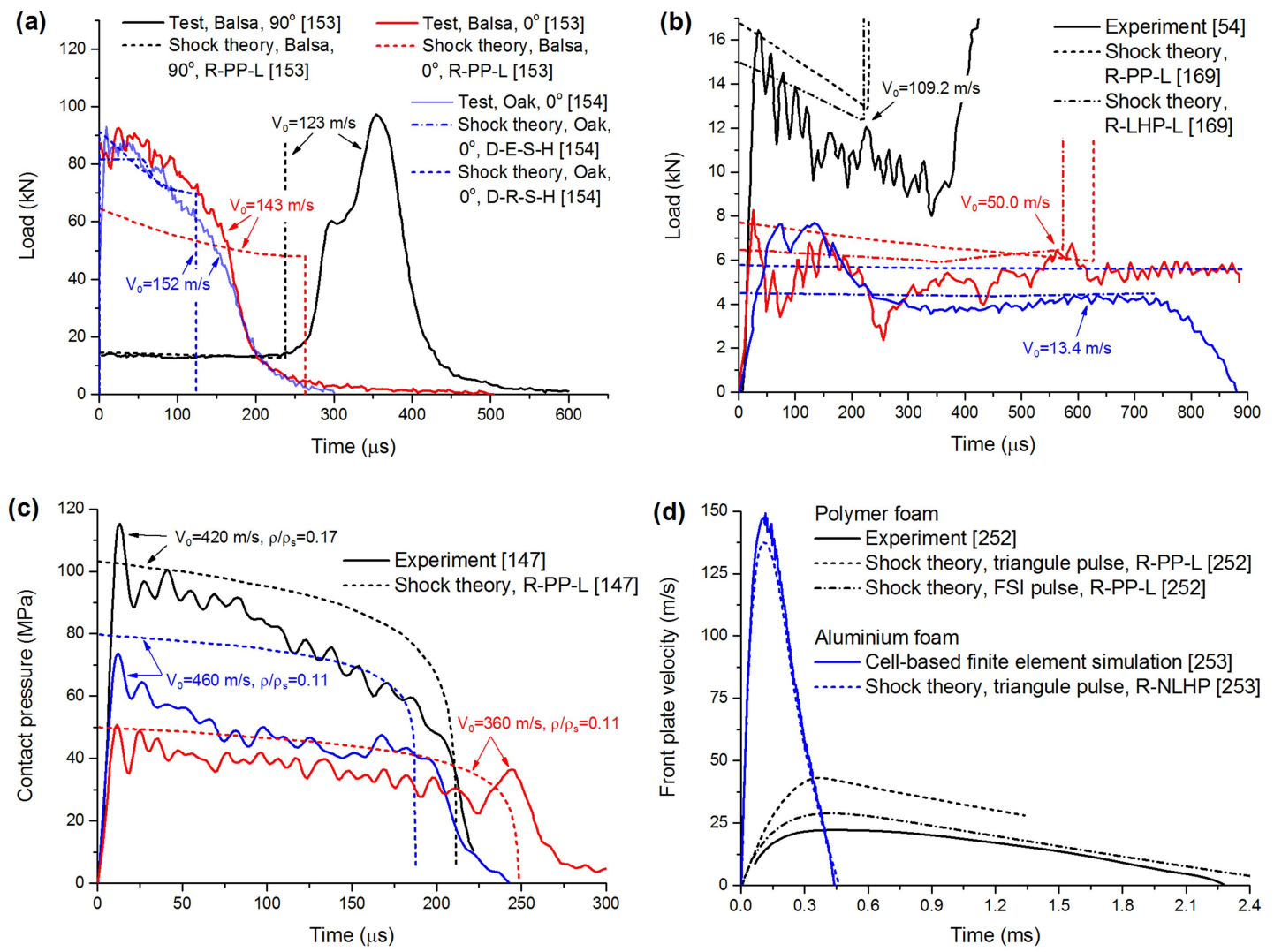

Fig. 35. Comparison between the analytical predictions of shock model and the observations from experimental tests and mesoscopic cell-based numerical simulations: (a) direct impact of wood (Scenario 1; $0^{\circ}$ and $90^{\circ}$ indicate "along grain" and "across grain", respectively) [153, 154]; (b) direct impact of aluminium Hydro/Cymat foam (Scenario 1) [54, 169]; (c) Taylor impact of aluminium Alporas foam (Scenario 3; rigid wall is unsupported) [147]; (d) blast loading of aluminium and polymer foams (Scenario 4; FSI: fluid-solid interaction) [252, 253].

In the theoretical framework of continuum mechanics, unified 1D shock models have been developed by Karagiozova et al. [172, 255, 260-262] and Zheng et al. [106, 169] mainly for the first two loading scenarios shown in Table 3. Karagiozova et al.'s analysis is based on a nonlinear strain-hardening material model, i.e. Eq. (23), with consideration of the reflection of/stress waves at the support end, the deformation of striker and the double layer configuration of cellular materials. The analytical prediction is compared with the continuum-based numerical simulation and the analytical solution using R-PP-L idealisation. Zheng et al.'s analysis is based on an arbitrary plastic hardening constitutive model, and specifically, R-LHP-L and power-law hardening, i.e. Eq. (24), are considered. The analytical 
prediction is validated by experimental tests and cell-based numerical simulations for aluminium foams, and also compared with R-PP-L based prediction. These unified shock models are applicable to strain-rate insensitive cellular materials and capable of predicting dynamic response to impact or blast load with various intensities. The main limitations of Karagiozova et al.'s and Zheng et al.'s analyses are that, (1) quasi-static stress-strain relation is used, which has been shown different from the Hugoniot relation of shock stress-strain state (Section 2.3.1); (2) smooth stress-strain function is assumed, and macroscopic plastic wave is allowed to propagate with relatively small magnitude of discontinuity $\left(\varepsilon_{\mathrm{b}}-\varepsilon_{\mathrm{a}}<\varepsilon_{\mathrm{d} 0}-\varepsilon_{\mathrm{c} 0}\right.$ and $\left.\sigma_{\mathrm{b}}-\sigma_{\mathrm{a}}<\sigma_{\mathrm{d} 0}-\sigma_{\mathrm{c} 0}\right)$, without considering the effect of local-softening induced stress oscillation in the plateau stage which is also associated with mesoscopic structural heterogeneity in foams (Fig. 4, 6, 25, 26 and 27). The first limitation has been overcome by Zheng et al. [149] recently, while the second limitation has not been carefully addressed or overcome up to date. In addition to its theoretical importance, the model's complexity and input parameters should be minimised in practical applications in order to reduce the time and cost of engineering analysis and design. Therefore, further studies on the sensitivity of prediction accuracy to material model selection (e.g. quasi-static stress-strain relation, rate-dependent plasticity and Hugoniot relation of shock stress-strain state) and alternative theoretical framework (e.g. generalised continuum mechanics [263-266]) are still needed for different types of cellular materials.

\subsubsection{General macroscopic models}

The yielding condition and plastic flow of cellular materials are complicated under multiaxial compression or combined loading. The prediction accuracy of structural response to complex loads largely depends on the constitutive model used in analysis. Uniaxial compression tests are most commonly used to obtain the yielding and hardening parameters required in plasticity models for cellular materials. They are also widely used to evaluate the strain-rate effect and shock state equations.

Since cellular materials are generally hydrostatically compressible, classical plasticity theory (e.g. von Mises theory) for incompressible material cannot describe well the plastic behaviour of cellular materials. An early attempt to develop yielding criterion for foams was made by Gibson et al. [267]. They considered bending and axial deformation of base material in an idealised cubic unit cell to predict the yielding of foam in a multiaxial stress state. In their model (also known as GAZT model), the deviatoric stress plays dominant roles although hydrostatic stress can also cause yielding. Abrate [268] summarised the yielding criteria for 
cellular materials reported in the literature and categorised them into four groups according to their mathematical expressions, which are listed in Table 4. The yield surface in stress space of Mises equivalent stress $\left(\sigma_{\mathrm{e}}\right)$ versus mean stress $\left(\sigma_{\mathrm{m}}\right)$ can be straight line (category 1), parabolic curve (categories 2 and 4), or elliptical curve (category 3). As the yield surfaces of these categories are symmetric with respect of $\sigma_{\mathrm{e}}$, these criteria imply an identical yield strength for tension and compression. A more general form of yield function can be expressed as

$$
\Phi=A_{1} \sigma_{\mathrm{e}}+A_{2} \sigma_{\mathrm{e}}^{2}+B_{1} \sigma_{\mathrm{m}}+B_{2} \sigma_{\mathrm{m}}^{2}-Y
$$

where $A_{1}, A_{2}, B_{1}$ and $B_{2}$ are material constants. The yielding criterion is $\Phi=0$. In most cases, Eq. (41) without more higher-order terms is adequate to determine the yield surface for a hydrostatically compressible material. For instance, the well-known Drucker-Prager criterion [269] widely used in soil mechanics is simply the linear form of Eq. (41).

Table 4 Four categories of yielding criterion for isotropic cellular material [268]

\begin{tabular}{cccc}
\hline Category & Mathematical form & Boundary shape in $\sigma_{\mathrm{e}}-\sigma_{\mathrm{m}}$ plane & Example \\
\hline 1 & $\sigma_{\mathrm{e}}=a_{0}+a_{1} \sigma_{\mathrm{m}}$ & Straight line & Ref. [270] \\
2 & $\sigma_{\mathrm{e}}=b_{0}+b_{1} \sigma_{\mathrm{m}}^{2}$ & Parabolic curve & Ref. [267] \\
3 & $\sigma_{\mathrm{e}}^{2}=c_{0}+c_{1} \sigma_{\mathrm{m}}^{2}$ & Elliptical curve & Ref. [60] \\
4 & $\sigma_{\mathrm{e}}^{2}=d_{0}+d_{1} \sigma_{\mathrm{m}}$ & Parabolic curve & Ref. [271] \\
\hline
\end{tabular}

Note: $\sigma_{\mathrm{m}}$ is mean stress; $\sigma_{\mathrm{e}}$ is Mises equivalent stress; other coefficients are material constants.

Examples of typical plasticity models for cellular materials are listed in Table 5, in which the yield functions all satisfy Eq. (41), although with some difference in the expression of constants. As aforementioned, GAZT model is based on mechanics analysis using one unit cell, so it can somehow reflect the relationship between mesoscopic deformation mechanism and macroscopic plastic behaviour. In contrast, the model of Zhang et al. [272] is merely phenomenological, and it is capable of incorporating the asymmetry between compression and tension (i.e. $x_{0}$ determines the elliptical centre). Miller's model [273] has certain physical basis since it can be reduced to GAZT model when $\gamma=0, \lambda=0.81 \rho / \rho_{\mathrm{s}}$ and $d=\sigma_{\mathrm{c} 0}$, but the physical meaning of the additional term (when $\gamma \neq 0$ ) is not clear. From macroscopic 
point of view, Deshpande and Fleck's model [60] can be correlated with strain energy of a cellular material. The strain energy density is composed of two parts associated with distortional and volumetric deformation, respectively, which can be expressed in terms of $\sigma_{\mathrm{e}}$ and $\sigma_{\mathrm{m}}$ as follows [268]

$$
U=\frac{1}{2} \sigma_{i j} \varepsilon_{i j}=\frac{1}{2 \bar{E}}\left[\sigma_{\mathrm{e}}^{2}+\beta^{2} \sigma_{\mathrm{m}}^{2}\right]
$$

where $\beta^{2}=4.5(1-2 v) /(1+v)$ and $\bar{E}=1.5 E /(1+v)$, with $E$ and $v$ being Young's modulus and Poisson's ratio, respectively. Note that in the yield function of Deshpande and Fleck's model [60], we have $\alpha^{2}=4.5\left(1-2 v_{\mathrm{p}}\right) /\left(1+v_{\mathrm{p}}\right)$ which is same as $\beta^{2} /$ if elastic Poisson's ratio $v$ is replaced by plastic Poisson's ratio $v_{\mathrm{p}}$.

Many studies have shown that the yield surfaces described by the plasticity models summarised in Table 5 are in reasonably good agreement with experimental results for multiaxial loading [14, 60, 62, 272, 274, 275] (e.g. Fíg. 39b). However, it seems that the phenomenological models fit experimental data better than the mechanism-based GAZT model which overestimates hydrostatic strength, and this overestimation is believed due to the bending deformation induced by mesoscopic structural imperfections, rather than the stretch/contraction assumed in the GAZT model [62, 267, 275].

The strain hardening can be modelled using different approaches. For instance, Zhang et al. [272] used plastic volumetric strain to determine the strain hardening of a cellular material. Deshpande and Fleck [60] defined an equivalent plastic strain as hardening variable. For large plastic deformation, Miller [273] pointed out that the hardening in the plateau stage should be determined by deviatoric strain of the cellular material (or more in essence, the shear deformation of the base material), but in the densification stage the volumetric strain plays more important roles as all cells are crushed and the compaction of the cellular material gradually dominates. Therefore, two separable hardening functions represented by different hardening variables were proposed by Miller [273].

The flow potential also has various forms. The plastic flow is associated with yield function in Miller's model [273] and Deshpande and Fleck's model [60], whereas a nonassociated law is used in the model by Zhang et al. [272]. The advantage of nonassociated flow potential is that a properly simplified function can be chosen when yield function is too complex, which can simplify the solution. For instance, Zhang et al. [272] chose a potential function as $F=\sqrt{\beta \sigma_{\mathrm{m}}^{2}+\sigma_{\mathrm{e}}^{2}}$ to account for both volumetric flow and shear flow, and they 
further demonstrated that this function implies that a plastic strain is directly proportional to the principal stress when $\beta=9 / 2$. Some experimental observations also support the choice of $\beta=9 / 2$, e.g. the compression of some cellular materials in one principal direction causes negligible plastic deformation in other transverse directions, i.e. plastic Poisson's ratio is close to zero [60-62].

Table 5 Typical plasticity models for cellular materials subjected to complex loading

\begin{tabular}{|c|c|c|c|}
\hline $\begin{array}{l}\text { Model } \\
\text { (Ref.) }\end{array}$ & Yield surface $\Phi=0$ & $\begin{array}{l}\text { Hardening } \\
\text { formulation }\end{array}$ & $\begin{array}{l}\text { Flow of plastic strain } \\
\dot{\varepsilon}_{i j}^{\mathrm{p}}=\dot{\lambda} \frac{\partial F}{\partial \tau}\end{array}$ \\
\hline [267] & $\Phi=\frac{\sigma_{\mathrm{e}}}{\sigma_{\mathrm{c} 0}}+0.81\left(\frac{\rho}{\rho_{\mathrm{s}}}\right)\left(\frac{\sigma_{\mathrm{m}}}{\sigma_{\mathrm{c} 0}}\right)^{2}-1$ & N/A & \\
\hline [272] & $\Phi=\frac{\left[\sigma_{\mathrm{m}}-x_{0}\right]^{2}}{a}+\frac{\sigma_{\mathrm{e}}^{2}}{b}-1$ & $\begin{array}{l}a=H_{2}\left(\varepsilon_{\mathrm{v}}^{\mathrm{p}}\right) \\
b=H_{3}\left(\varepsilon_{\mathrm{v}}^{\mathrm{p}}\right)\end{array}$ & $F=\sqrt{\beta \sigma_{\mathrm{m}}^{2}+\sigma_{\mathrm{e}}^{2}}$ \\
\hline [273] & $\Phi=\sigma_{\mathrm{e}}+\gamma \sigma_{\mathrm{m}}+\frac{\lambda \sigma_{\mathrm{m}}^{2}}{d}-d$ & & $F=\Phi$ \\
\hline$[60]$ & $\Phi=\sqrt{\frac{1}{1+(\alpha / 3)^{2}}\left[\sigma_{\mathrm{e}}^{2}+\alpha^{2} \sigma_{\mathrm{m}}^{2}\right]}-$ & $Y=H\left(\varepsilon_{\mathrm{i}}^{\mathrm{p}}\right)$ & $F=\Phi$ \\
\hline
\end{tabular}

Note: $\sigma_{\mathrm{m}}$ is mean stress; $\sigma_{\mathrm{e}}$ is Mises equivalent stress; $\epsilon_{\mathrm{v}}$ is total volumetric strain; $\epsilon_{\mathrm{v}}^{\mathrm{p}}$ is plastic volumetric strain; $\epsilon_{\mathrm{i}}^{\mathrm{p}}$ is Mises equivalent plastic strain; $\sigma_{\mathrm{c} 0}$ is uniaxial plastic collapse initiation stress; $\rho / \rho_{\mathrm{s}}$ is relative density; $Y$ is yield stress; other coefficients are material parameters. The specific forms of hardening function can be found in Refs. [60, 272, 273].

Some plasticity models have been incorporated into commercial FE codes such as Abaqus and LS-dyna for numerical computation. For instance, Abaqus has two crushable foam models with volumetric hardening and isotropic hardening, of which the volumetric hardening model is similar to the model by Zhang et al. [272], whereas the isotropic hardening model is based on Deshpande and Fleck's model [60]. LS-dyna also provides extensive constitutive models for cellular materials, including NO. 5, 14, 26, 38, 53, 57, 61, 62, 63, 73, 75, 85 and 126 [276]. Hanssen [246] selected NO. 26, 63, 75 and 126 material models of LS-dyna to examine their performance in various loading cases such as compression, indentation, diagonal loading, bending of foam-filled beam, and crushing of foam-filled extrusions. The No. 26 and No. 126 models are both based on a yield criterion that a material yields when one 
stress component exceeds its corresponding yield stress. However, for the former the hardening is determined by volumetric strain, while for the latter the hardening for each yield stress component is associated with the corresponding strain component. The No. 63 model defines the yielding using largest principle stress and the hardening depends on volumetric strain. The No. 75 model is similar to the model by Zhang et al. [272]. The numerical and experimental indentation results are shown in Fig. 36. The No. 63 and No. 75 models perform better than the other two, but they still suffer discrepancy with experimental observations, e.g. the indented cube exhibits inward bending at the outer edges when No. 63 model is used and the elements move sidewards for No. 75 model. The results of different validation tests reveal that all of the selected models have their own drawbacks depending on loading cases, and only limited accuracy can be achieved [246]. Therefore, these macroscopic material models should be used with care. 

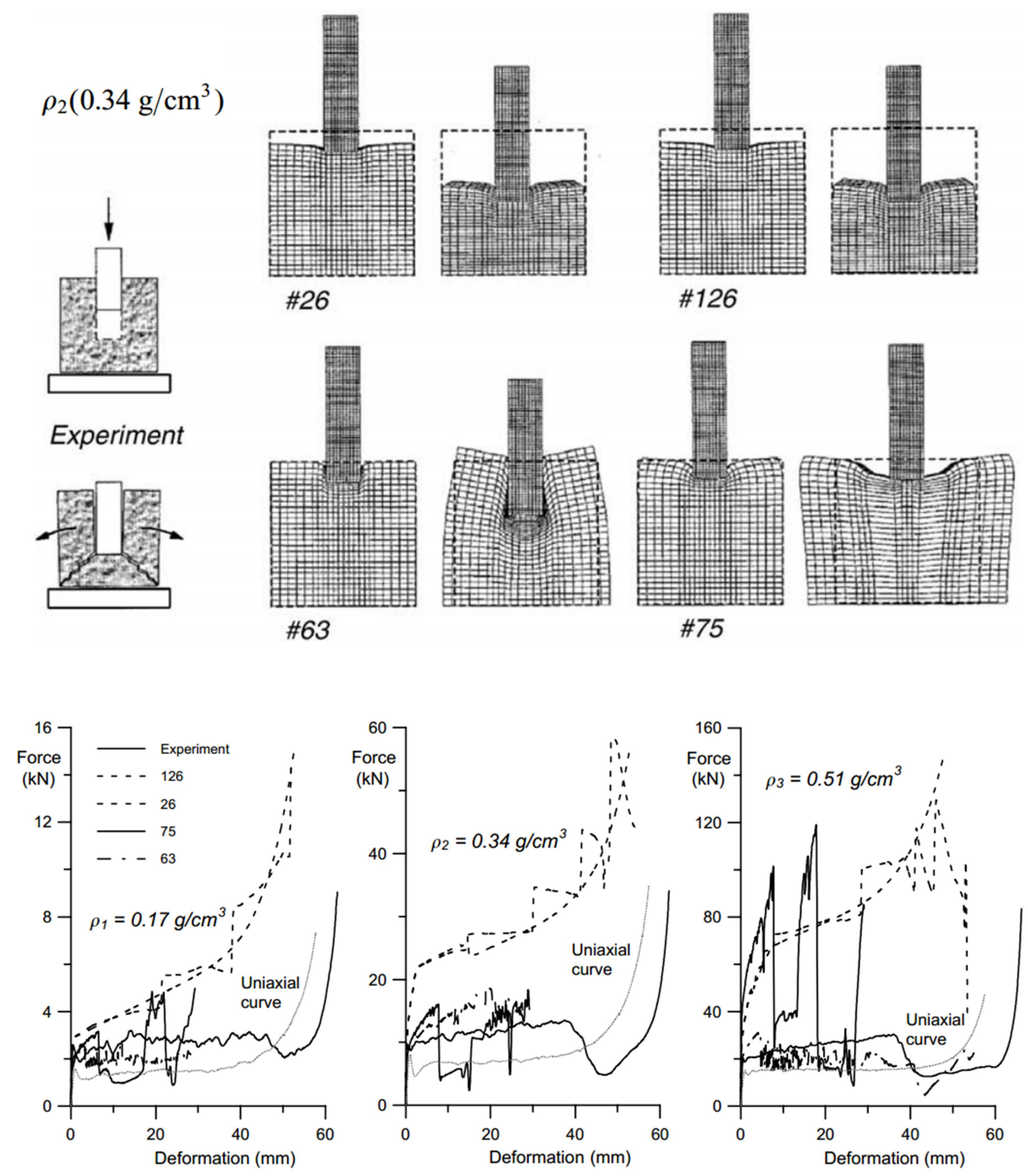

Fig. 36. Validation of different constitutive material models using indentation test [246]. Top: deformation configurations; Bottom: force-deformation plots. The label number of material model refers to Ls-dyna material library. Descriptions of the models can be found in the texts and more details see Ref. [246].

Uniaxial compression tests can be used to determine the material parameters in the aforementioned plasticity models. For instance, when the uniaxial plastic collapse initiation stress is obtained, the yield surface defined by Gibson et al. (1989) can be readily determined. However, as cellular materials are generally compressible, hydrostatic compression tests may be also necessary to obtain additional material parameters. For instance, the material parameter $\alpha$ in the yield function (see Table 5) proposed by Deshpande and Fleck [60] is equal to $\sqrt{1 /\left(x^{2}-1 / 9\right)}$ where $x$ is the yield strength ratio between hydrostatic and uniaxial 
compressions, and the material parameter $Y$ is equal to compressive strength (e.g. $0.3 \%$ offset yield point). Alternatively, the $\alpha$ value also can be determined from plastic Poisson's ratio in uniaxial compression [60]. In other words, all material parameters of a plasticity model [60] used to describe multiaxial plastic behaviour can be determined from one uniaxial compression test.

In structural response analysis, an appropriate description of constitutive behaviour of cellular materials is needed to establish analytical and numerical models for engineering structures such as sandwich beams or plates with cellular core materials. For instance, Qin and Wang [277-280] proposed a unified yield criterion to obtain the analytical solutions of the dynamic response of sandwich structures under a variety of quasi-static and dynamic loading conditions. The general plasticity models for cellular materials are used not only in the numerical study of uniaxial compression $[172,210]$, but also in the impact simulation of complicated structures such as foam-cored sandwich beams and panels [280-285], as shown in Fig. 37. In most cases, these analytical and numerical models are used without consideration of strain-rate or shock effect, which are acceptable in some circumstances, but such neglect may introduce unacceptable errors for some strain-rate sensitive cellular materials [125] (see Sections 2.2 and 2.3 for obseryed loading rate effect). In particular, high accuracy of prediction for dynamic compression is strictly required in some safety critical applications, e.g. nuclear components $[125,286]$.

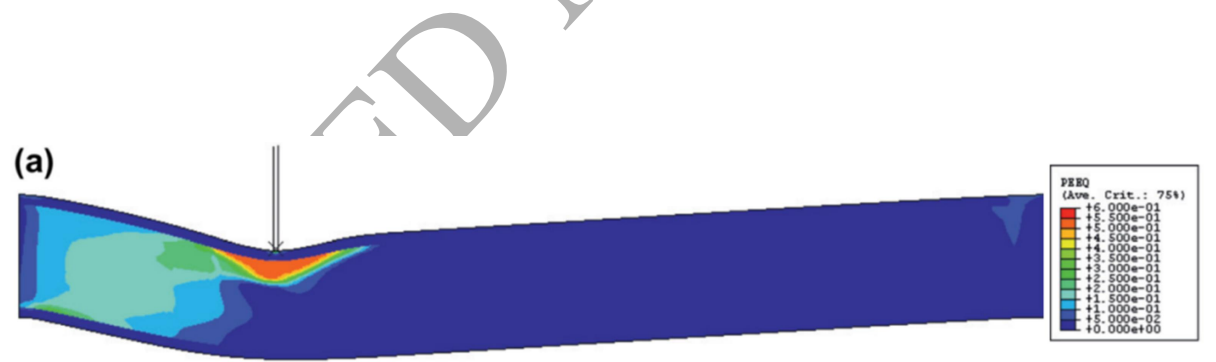

(b)

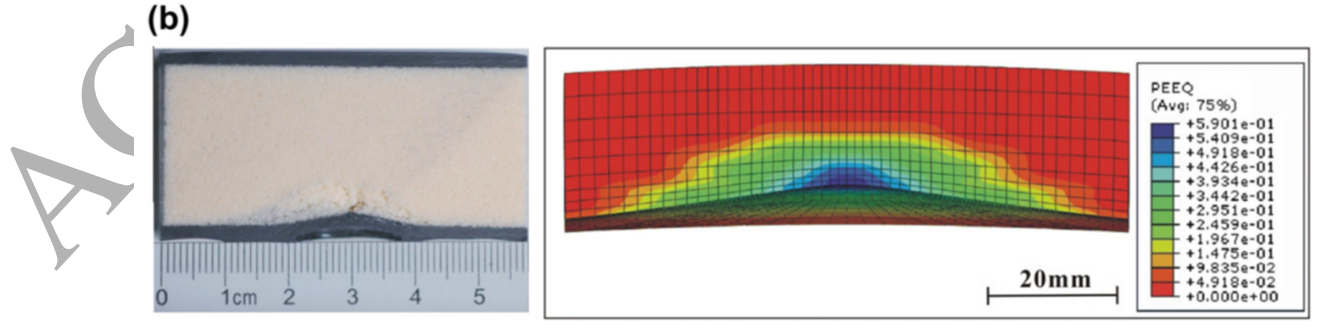

Fig. 37. Continuum-based FE impact simulations: (a) distribution of 2D equivalent plastic strain in a foam-cored sandwich beam under low-velocity impact [287]; (b) comparison of foam crushing area between impacted specimen (left) and 3D numerical result (right) for a foam-cored sandwich panel [288]. 
Extensive experimental test data are needed to establish the rate-dependence of yield surface of a strain-rate sensitive cellular material. German BAM [125] identified significant discrepancies between the numerical simulations neglecting strain-rate effect and the actual dynamic behaviour of impact limiters made of polyurethane foam for transport and storage of highly radioactive waste. They thus launched an ENREA project comprising experimental tests of over 1000 specimens for wood, polyurethane foam and polymer concrete under compression with loading speed ranging from $0.02 \mathrm{~mm} / \mathrm{s}$ to $3 \mathrm{~m} / \mathrm{s}$. Their compressive experimental results for polyurethane foam, as shown in Fig. 38 (a), demonstrate that a proper modelling method (e.g. Abaqus crushable foam model with isotropic hardening and tabular rate-dependent scaling factors) can describe well the rate-dependence of foam plasticity. However, the Cowper-Symonds relation was shown unsatisfactory for the foams tested. Zhang et al. [122] incorporated the strain-rate sensitivity (described by) Eq. 29) into the constitutive model (Zhang et al. [272], see Table 5) for polymer foams and they observed good agreement with compressive experimental results (Fig. 38b),
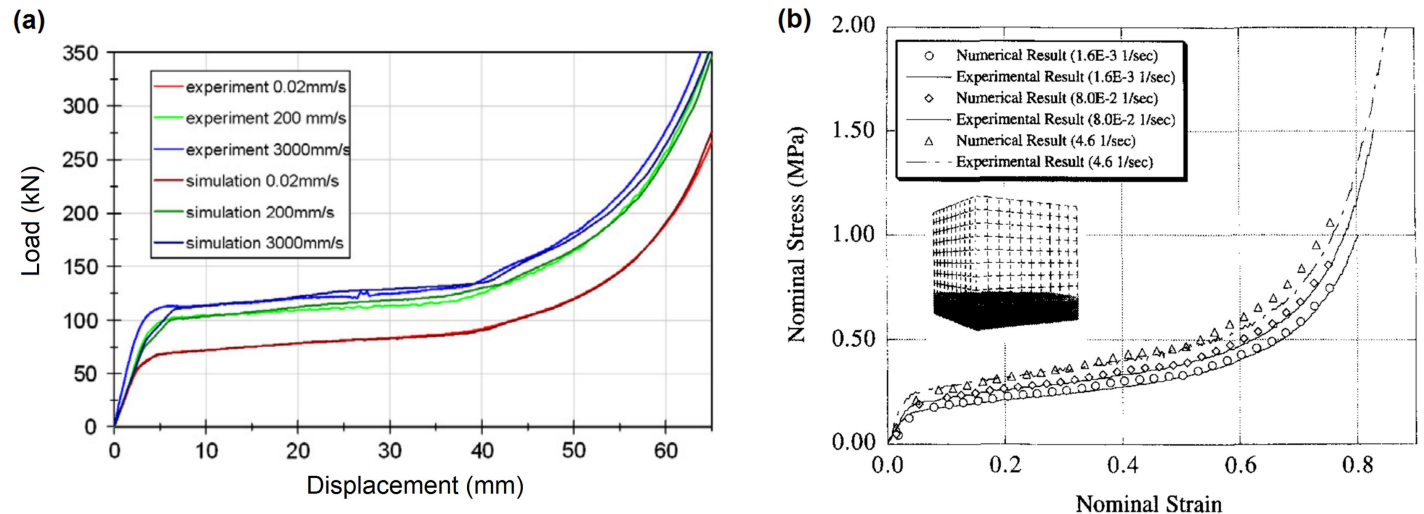

Fig. 38. Comparison between experiment and simulation incorporating strain-rate effect in plasticity models for polymer foams: (a) load-displacement curves [125]; (b) stress-strain curves [122].

For multi-axial compression, Chung and Waas [289, 290] investigated the in-plane biaxial static and dynamic compressions of polycarbonate honeycombs and observed complex expansion of the maximum stress envelop, as shown in Fig. 39 (a). For dynamic combined loading cases, Hou et al. [291, 292] found that the crush envelope expands isotropically for aluminium honeycombs subjected to combined compression-shear loading (out-of-plane loading). Zhou et al. [274] conducted combined compression-shear test for three types of 
foams and found that the initial yield surface exhibits an almost isotropic expansion with increasing loading rate, as shown in Fig. 39 (b), wherein the experimental results are also compared with yield criteria reported in Refs. $[60,267,273]$ (see Table 5 for expressions of yield criteria). However, the dynamic multiaxial loading test results are very limited in the literature. Therefore, there is a need to produce high quality benchmark experimental data under complex stress state, in both static and dynamic loading conditions, together with relatively complete material data for the purpose of calibration and validation of numerical models.
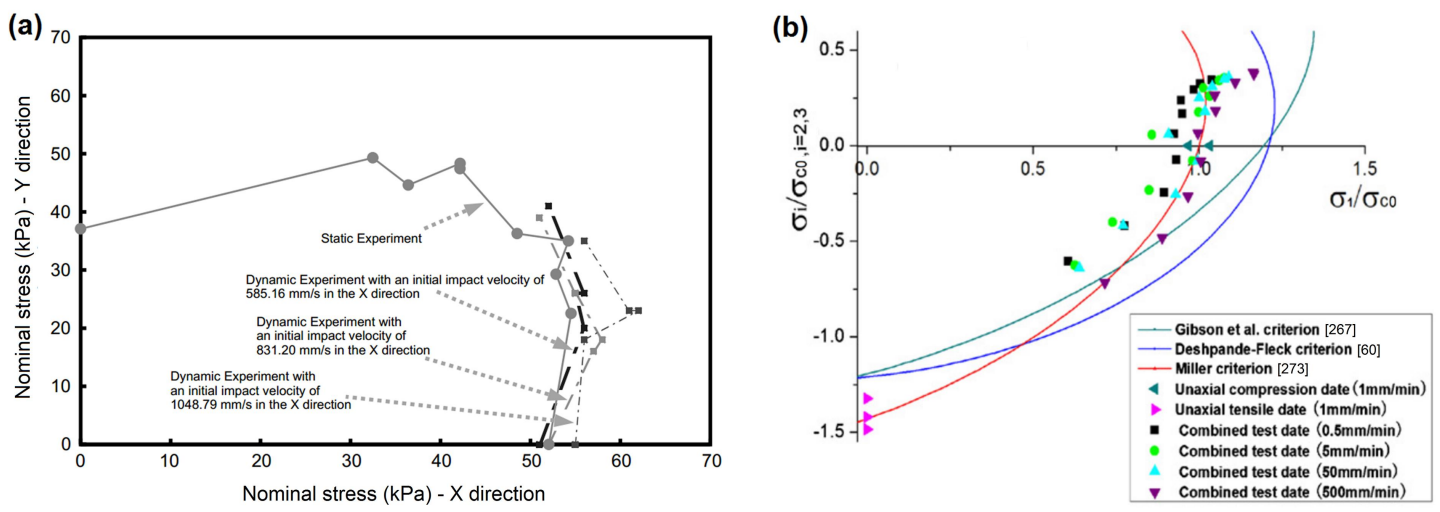

Fig. 39. (a) Maximum stress envelopes obtained from static and dynamic combined compression tests for polycarbonate honeycombs [289]; (b) yield surfaces for combined loading tests of aluminium alloy foams under different loading rates [274].

For the transitional dynamic regime, the application of Eqs. (28)-(31) can be extended to multiaxial loading cases when equivalent stress and strain are used to take account of strain-rate effect. However, it should be noted that the difference between the shock equation-of-state (e.g. stress-strain Hugoniot) and the quasi-static or transitional dynamic stress-strain constitutive relation has not been fully clarified in previous studies. The strategy to apply the dynamic constitutive relation and the shock Hugoniot relation to impact or blast analysis is still an open topic.

\section{Concluding remarks}

The dynamic compressive behaviour of cellular materials is described and discussed with regard to observations, mechanisms and modelling in this review. A general conclusion of the strain-rate sensitivity of cellular materials has not been drawn, as the governing mechanisms are sensitive to base material properties and cell structure characteristics which are 
determined by specific manufacturing routines for different types of cellular materials. Despite the scatter in experimental data due to either inherent scatter among tested samples or the limitation of experimental techniques, the plastic properties of some types of cellular materials, such as steel foams, aluminium Alporas foam, Balsa wood (loaded along grain), honeycombs (out-of-plane compression) and many polymer foams, have been widely recognised as rate dependent. The rate dependence of these cellular materials in the transitional dynamic regime at intermediate loading rates mainly arises from the microscopic strain-rate sensitivity of base material, micro-inertia or gas pressurisation, or their combination. Furthermore, the effects of these micro- and meso-scale factors can be enhanced by combining with other factors. For instance, the combination of micro-inertia and strain hardening of base material can significantly increase the plateau stress of honeycombs under dynamic out-of-plane compression.

At sufficiently high loading speed, shock initiates in most of eellular materials, which is characterised by the distinctive cell deformation, i.e. the formation and propagation of macroscopic discontinuity wave front from loading end, in contrast to the randomly distributed collapse or crush bands formed in quasi-static and transitional dynamic regimes. The jumps of some physical quantities (e.g. stress, strain and energy) across the shock front are commonly perceived as shock enhancement. The dominant axial inertial resistance and the cell interactions at the meso-scale under high speed impact are linked to the occurrence of the compaction shock in cellular materials. Usually, a sufficiently high loading speed or intensity is necessary for the initiation of the compaction shock, and a variety of methods can be applied to predict the critical impact velocity for the shock initiation, among which the method based on Hugoniot relation was shown to provide better prediction. Equally importantly, shock speed has a linear relation with loading speed, and shock compression leads to distinctive load transmission and stress-strain states.

Mesoscopic modelling proves useful to identify and quantify the effects of different mechanisms. Mass-spring models are attractive due to their simplicity, but they have been rarely validated by experiments and have disadvantages to handle shock problem. Cell-based analytical models are valuable to gain general insights, but they have suffered difficulty to capture the complicated compressive behaviour of stochastic foams/wood and have significant limitation in dynamics analysis of honeycombs subjected to large deformation. Therefore, extensive cell-based numerical models have been developed and employed to analyse the dynamic compressive behaviours of different types of cellular materials. The emerging image-based modelling is particularly useful for the investigation of a specific 
cellular material imaged, as the actual cell structure is incorporated in the modelling. The models based on idealised cell structures are more powerful in terms of structural parametric sensitivity analysis. The mesoscopic modelling analyses substantially improve our understanding of the mechanisms controlling the dynamic compressive behaviour of cellular materials. However, a macroscopic continuum-based modelling approach, with satisfactory accuracy for both uniaxial and multiaxial loadings, is preferred for analysis of dynamic response of engineering structures (e.g. foam-filled sandwich plates) to complex loads, for the sake of simplifying problems and reducing computational cost/time. It has been demonstrated that the correct application of conservation laws, and the accurate description of constitutive relation, shock stress-strain states and dynamic loading, are crucial for the success in continuum-based modelling.

Some outstanding issues have been also identified and addressed, which may need further studies. First, the investigation of base material behaviour has not been sufficiently endeavoured yet; in particular, the strain-rate sensitivity of base material is believed dependent on the manufacturing process, but it still lacks dynamic test results directly for the base material. Second, the strain-rate effect in the transitional dynamic regime and the Hugoniot relation for shock should be properly incorporated in continuum-based models for both uniaxial and multiaxial compressions (or other types of loads). Third, fracture behaviour and its effect on dynamic compression have been rarely examined in experiments and modelling; for closed-cell foams, cracking of cell walls may affect gas leaking and hence the contribution of gas pressure to the compressive resistance. Fourth, the mesoscopic stress wave propagation in cellular materials has been much less studied in comparison with the macroscopic discontinuity wave (e.g. shock), and the related mesoscopic mechanisms are still largely unclear; an improved understanding of the mesoscopic load transmission in the cell structure will shed further light on the underpinning mechanism of shock initiation and propagation. Fifth, the interaction of different mechanisms has not been adequately understood, particularly for shock which may involve all of the aforementioned micro- and meso-scale/mechanisms. Finally, dynamic compressive behaviour of novel cellular materials, e.g. functionally graded [82-84, 293, 294] and hierarchical [85-87] cellular materials, and cellular composites [295-297], as well as hypervelocity compressive behaviour [9, 10] and multiaxial compression (or combined loading), deserve further research.

Acknowledgements: The authors are grateful to the anonymous reviewers for their constructive comments. Supports from State Key Laboratory of Explosion Science and 
Technology (ZDKT11-03) and National Natural Science Foundation of China (11521062) are acknowledged.

\section{References}

[1] L.J. Gibson, M.F. Ashby, Cellular solids: structure and properties, 2 ed., Cambridge University Press, 1997.

[2] Y.H.M. Amran, N. Farzadnia, A.A. Abang Ali, Properties and applications of foamed concrete; a review, Construction and Building Materials, 101 (2015) 990-1005.

[3] M.F. Ashby, A.G. Evans, N.A. Fleck, L.J. Gibson, J.W. Hutchinson, H.N.G. Wadley, Metal foams: a design guide, Elsevier, 2000.

[4] J. Banhart, Manufacture, characterisation and application of cellular metals and metal foams, Progress in Materials Science, 46 (2001) 559-632.

[5] J. Banhart, Aluminum foams: On the road to real applications, MRS bulletin, 28 (2003) 290-295.

[6] B.A. Gama, T.A. Bogetti, B.K. Fink, C.-J. Yu, T. Dennis Claar, H.H. Eifert, J.W. Gillespie Jr, Aluminum foam integral armor: a new dimension in armor design, Composite Structures, 52 (2001) 381-395.

[7] L.J. Gibson, M.F. Ashby, B.A. Harley, Cellular materials in nature and medicine, Cambridge University Press, 2010.

[8] L.P. Lefebvre, J. Banhart, D.C. Dunand, Porous Metals and Metallic Foams: Current Status and Recent Developments, Advanced Engineering Materials, 10 (2008) 775-787.

[9] S. Ryan, E.L. Christiansen, Hypervelocity impact testing of advanced materials and structures for micrometeoroid and orbital debris shielding, Acta Astronaut, 83 (2013) 216-231.

[10] S. Ryan, T. Hedman, E.L. Christiansen, Honeycomb vs. foam: Evaluating potential upgrades to ISS module shielding, Acta Astronaut, 67 (2010) 818-825.

[11] H. Zhou, G. Ma, J. Li, Z. Zhao, Design of Metal Foam Cladding Subjected to Close-Range Blast, Journal of Performance of Constructed Facilities, 29 (2015) 04014110.

[12] A.E. Simone, L.J. Gibson, Aluminum foams produced by liquid-state processes, Acta Materialia, 46 (1998) 3109-3123. 
[13] I. Elnasri, S. Pattofatto, H. Zhao, H. Tsitsiris, F. Hild, Y. Girard, Shock enhancement of cellular structures under impact loading: Part I Experiments, Journal of the Mechanics and Physics of Solids, 55 (2007) 2652-2671.

[14] D. Ruan, G. Lu, L.S. Ong, B. Wang, Triaxial compression of aluminium foams, Composites Science and Technology, 67 (2007) 1218-1234.

[15] R. Liu, A. Antoniou, A relationship between the geometrical structure of a nanoporous metal foam and its modulus, Acta Materialia, 61 (2013) 2390-2402.

[16] M.A. Hasan, A. Kim, H.J. Lee, Measuring the cell wall mechanical properties of Al-alloy foams using the nanoindentation method, Composite Structures, 83 (2008) 180-188.

[17] A.H. König, H. Doleisch, A. Kottar, B. Kriszt, E. Gröller, AlVis - An Aluminium-Foam Visualization and Investigation Tool, in: W.C. de Leeuw, R. van Liere (Eds.) Data Visualization 2000: Proceedings of the Joint EUROGRAPHICS and IEEE TCVG Symposium on Visualization in Amsterdam, The Netherlands, May 29-30, 2000, Springer Vienna, Vienna, 2000, pp. 229-238.

[18] A.F. Bastawros, H. Bart-Smith, A.G. Evans, Experimental analysis of deformation mechanisms in a closed-cell aluminum alloy foam, Journal of the Mechanics and Physics of Solids, 48 (2000) 301-322.

[19] A.F. Bastawros, A.G. Evans, Deformation Heterogeneity in Cellular Al Alloys, Advanced Engineering Materials, 2 (2000) 210-214.

[20] Z. Zou, S.R. Reid, P.J. Tan, S. Li, J.J. Harrigan, Dynamic crushing of honeycombs and features of shock fronts, International Journal of Impact Engineering, 36 (2009) 165-176.

[21] Y. Sun, X. Zhang, Z. Shao, Q.M. Li, Image-based correlation between the meso-scale structure and deformation of closed-cell foam, Materials Science and Engineering: A, 688 (2017) 27-39.

[22] S. Liao, Z. Zheng, J. Yu, Dynamic crushing of 2D cellular structures: Local strain field and shock wave velocity, International Journal of Impact Engineering, 57 (2013) 7-16.

[23] S. Liao, Z. Zheng, J. Yu, On the local nature of the strain field calculation method for measuring heterogeneous deformation of cellular materials, International Journal of Solids and Structures, 51 (2014) 478-490.

[24] I. Jeon, T. Asahina, K.-J. Kang, S. Im, T.J. Lu, Finite element simulation of the plastic collapse of closed-cell aluminum foams with X-ray computed tomography, Mechanics of Materials, 42 (2010) 227-236.

[25] Y. Sun, Q.M. Li, S.A. McDonald, P.J. Withers, Determination of the constitutive relation and critical condition for the shock compression of cellular solids, Mechanics of Materials, 99 (2016) 26-36. 
[26] Y. Sun, Q.M. Li, T. Lowe, S.A. McDonald, P.J. Withers, Investigation of strain-rate effect on the compressive behaviour of closed-cell aluminium foam by 3D image-based modelling, Materials \& Design, 89 (2016) 215-224.

[27] L.J. Gibson, The elastic and plastic behaviour of cellular materials, $\mathrm{PhD}$ thesis, University of Cambridge (1981).

[28] J.L. Yu, J.R. Li, S.S. Hu, Strain-rate effect and micro-structural optimization of cellular metals, Mechanics of Materials, 38 (2006) 160-170.

[29] R. Bouix, P. Viot, J.-L. Lataillade, Polypropylene foam behaviour under dynamic loadings: Strain rate, density and microstructure effects, International Journal of Impact Engineering, 36 (2009) 329-342.

[30] H. Yu, Z. Guo, B. Li, G. Yao, H. Luo, Y. Liu, Research into the effect of cell diameter of aluminum foam on its compressive and energy absorption properties, Materials Science and Engineering: A, 454-455 (2007) 542-546.

[31] H. Bart-Smith, A.F. Bastawros, D.R. Mumm, A.G. Evans, D.J. Sypeck, H.N.G. Wadley, Compressive deformation and yielding mechanisms in cellular $\mathrm{Al}$ alloys determined using X-ray tomography and surface strain mapping, Acta Materialia, 46 (1998) 3583-3592.

[32] A.E. Simone, L.J. Gibson, The effects of cell face curvature and corrugations on the stiffness and strength of metallic foams, Acta Materialia, 46 (1998) 3929-3935.

[33] A.E. Simone, L.J. Gibson, Effects of solid distribution on the stiffness and strength of metallic foams, Acta Materialia, 46 (1998) 2139-2150.

[34] Y. Sun, Q.M. Li, Effect of entrapped gas on the dynamic compressive behaviour of cellular solids, International Journal of Solids and Structures, 63 (2015) 50-67.

[35] L. Tang, X. Shi, L. Zhang, Z. Liu, Z. Jiang, Y. Liu, Effects of statistics of cell's size and shape irregularity on mechanical properties of 2D and 3D Voronoi foams, Acta Mechanica, 225 (2014) 1361-1372.

[36] V.S. Deshpande, M.F. Ashby, N.A. Fleck, Foam topology: bending versus stretching dominated arehitectures, Acta Materialia, 49 (2001) 1035-1040.

[37] N.A. Fleck, V.S. Deshpande, M.F. Ashby, Micro-architectured materials: past, present and future, Proceedings of the Royal Society A: Mathematical, Physical and Engineering Science, 466 (2010) 2495-2516.

[38] Y. Liu, X.-C. Zhang, The influence of cell micro-topology on the in-plane dynamic crushing of honeycombs, International Journal of Impact Engineering, 36 (2009) 98-109. 
[39] M. Alkhader, M. Vural, Mechanical response of cellular solids: Role of cellular topology and microstructural irregularity, International Journal of Engineering Science, 46 (2008) 1035-1051.

[40] C. Chen, T.J. Lu, N.A. Fleck, Effect of imperfections on the yielding of two-dimensional foams, Journal of the Mechanics and Physics of Solids, 47 (1999) 2235-2272.

[41] I. Jeon, T. Asahina, The effect of structural defects on the compressive behavior of closed-cell Al foam, Acta Materialia, 53 (2005) 3415-3423.

[42] A. Ajdari, H. Nayeb-Hashemi, A. Vaziri, Dynamic crushing and energy absorption of regular, irregular and functionally graded cellular structures, International Journal of Solids and Structures, 48 (2011) 506-516.

[43] K. Li, X.L. Gao, J. Wang, Dynamic crushing behavior of honeycomb structures with irregular cell shapes and non-uniform cell wall thickness, International Journal of Solids and Structures, 44 (2007) 5003-5026.

[44] X.-C. Zhang, Y. Liu, B. Wang, Z.-M. Zhang, Effects of defects on the in-plane dynamic crushing of metal honeycombs, International Journal of Mechanical Sciences, 52 (2010) 1290-1298.

[45] M.J. Silva, L.J. Gibson, The effects of non-periodic microstructure and defects on the compressive strength of two-dimensional cellular solids, International Journal of Mechanical Sciences, 39 (1997) 549-563.

[46] A. Ajdari, H. Nayeb-Hashemi, P. Canavan, G. Warner, Effect of defects on elastic-plastic behavior of cellular materials, Materials Science and Engineering: A, 487 (2008) 558-567.

[47] E. Wu, W.-S. Jiang, Axial crush of metallic honeycombs, International Journal of Impact Engineering, 19 (1997) 439-456.

[48] B. Hou, H. Zhao, S. Pattofatto, J.G. Liu, Y.L. Li, Inertia effects on the progressive crushing of aluminium honeycombs under impact loading, International Journal of Solids and Structures, 49 (2012) 2754-2762.

[49] D. Karagiozova, M. Alves, On the dynamic compression of cellular materials with local structural softening, International Journal of Impact Engineering, 108 (2017) 153-170.

[50] D. Karagiozova, Dynamic buckling of elastic-plastic square tubes under axial impact-I: stress wave propagation phenomenon, International Journal of Impact Engineering, 30 (2004) 143-166.

[51] D. Karagiozova, N. Jones, Dynamic buckling of elastic-plastic square tubes under axial impact-II: structural response, International Journal of Impact Engineering, 30 (2004) 167-192. 
[52] C.Q. Xi, Q.M. Li, Meso-scale mechanism of compaction shock propagation in cellular materials, International Journal of Impact Engineering, 109 (2017) 321-334.

[53] P. Wang, X. Wang, Z. Zheng, J. Yu, Stress Distribution in Graded Cellular Materials Under Dynamic Compression, Latin American Journal of Solids and Structures, 14 (2017) 1251-1272.

[54] P.J. Tan, S.R. Reid, J.J. Harrigan, Z. Zou, S. Li, Dynamic compressive strength properties of aluminium foams. Part I-experimental data and observations, Journal of the Mechanics and Physics of Solids, 53 (2005) 2174-2205.

[55] P.J. Tan, S.R. Reid, J.J. Harrigan, Z. Zou, S. Li, Dynamic compressive strength properties of aluminium foams. Part II-'shock' theory and comparison with experimental data and numerical models, Journal of the Mechanics and Physics of Solids, 53 (2005) 2206-2230.

[56] B.P. Neville, A. Rabiei, Composite metal foams processed through powder metallurgy, Materials \& Design, 29 (2008) 388-396.

[57] A. Rabiei, L.J. Vendra, A comparison of composite metal foam's properties and other comparable metal foams, Materials Letters, 63 (2009) 533-536.

[58] C.R. Calladine, R.W. English, Strain-rate and inertia effects in the collapse of two types of energy-absorbing structure, International Journal of Mechanical Sciences, 26 (1984) 689-701.

[59] Y.D. Liu, J.L. Yu, Z.J. Zheng, J.R. Li, A numerical study on the rate sensitivity of cellular metals, International Journal of Solids and Structures, 46 (2009) 3988-3998.

[60] V.S. Deshpande, N.A. Fleck, Isotropic constitutive models for metallic foams, Journal of the Mechanics and Physics of Solids, 48 (2000) 1253-1283.

[61] M.C. Shaw, T. Sata, The plastic behavior of cellular materials, International Journal of Mechanical Sciences, 8 (1966) 469-478.

[62] G, Gioux, T.M. McCormack, L.J. Gibson, Failure of aluminum foams under multiaxial loads, International Journal of Mechanical Sciences, 42 (2000) 1097-1117.

[63] H. Zhao, S. Abdennadher, On the strength enhancement under impact loading of square tubes made from rate insensitive metals, International Journal of Solids and Structures, 41 (2004) 6677-6697.

[64] V.S. Deshpande, N.A. Fleck, High strain rate compressive behaviour of aluminium alloy foams, International Journal of Impact Engineering, 24 (2000) 277-298. 
[65] H. Zhao, I. Elnasri, S. Abdennadher, An experimental study on the behaviour under impact loading of metallic cellular materials, International Journal of Mechanical Sciences, 47 (2005) 757-774.

[66] D. Ruan, G. Lu, B. Wang, T.X. Yu, In-plane dynamic crushing of honeycombs-a finite element study, International Journal of Impact Engineering, 28 (2003) 161-182.

[67] Z. Zheng, J. Yu, J. Li, Dynamic crushing of 2D cellular structures: A finite element study, International Journal of Impact Engineering, 32 (2005) 650-664.

[68] S. Lee, F. Barthelat, N. Moldovan, H.D. Espinosa, H.N.G. Wadley, Deformation rate effects on failure modes of open-cell Al foams and textile cellular materials, International Journal of Solids and Structures, 43 (2006) 53-73.

[69] A.T. Barnes, K. Ravi-Chandar, S. Kyriakides, S. Gaitanaros, Dynamic crushing of aluminum foams: Part I - Experiments, International Journal of Solids and Structures, 51 (2014) 1631-1645.

[70] G.W. Ma, Z.Q. Ye, Z.S. Shao, Modeling loading rate effect on crushing stress of metallic cellular materials, International Journal of Impact Engineering, 36 (2009) 775-782.

[71] L.-L. Wang, L.-M. Yang, Y.-Y. Ding, On the energy conservation and critical velocities for the propagation of a "steady-shock" wave in a bar made of cellular material, Acta Mechanica Sinica, (2013) 1-9.

[72] A.G. Hanssen, L. Enstock, M. Langseth, Close-range blast loading of aluminium foam panels, International Journal of Impact Engineering, 27 (2002) 593-618.

[73] Q.M. Li, H. Meng, Attenuation or enhancement - A one-dimensional analysis on shock transmission in the solid phase of a cellular material, International Journal of Impact Engineering, 27 (2002) 1049-1065.

[74] G. Ben-Dor, G. Mazor, O. Igra, S. Sorek, H. Onodera, Shock wave interaction with cellular materials Part II: open cell foams; experimental and numerical results, Shock Waves, 3 (1994) 167-179.

[75] O.E. Petel, S. Ouellet, A.J. Higgins, D.L. Frost, The elastic-plastic behaviour of foam under shock loading, Shock Waves, 23 (2013) 55-67.

[76] H. Zhou, Z. Zhao, G. Ma, Protection against blast load with cellular materials and structures, International Journal of Aerospace and Lightweight Structures 2(2012) 53-76.

[77] F. Zhu, C.C. Chou, K.H. Yang, Shock enhancement effect of lightweight composite structures and materials, Composites Part B: Engineering, 42 (2011) 1202-1211.

[78] M.F. Ashby, The properties of foams and lattices, Philosophical Transactions of the Royal Society of London. Series A, Mathematical and Physical Sciences, 364 (2006) 15-30. 
[79] T.A. Schaedler, W.B. Carter, Architected Cellular Materials, Annual Review of Materials Research, 46 (2016) 187-210.

[80] X.M. Qiu, J. Zhang, T.X. Yu, Collapse of periodic planar lattices under uniaxial compression, part I: Quasi-static strength predicted by limit analysis, International Journal of Impact Engineering, 36 (2009) 1223-1230.

[81] X.M. Qiu, J. Zhang, T.X. Yu, Collapse of periodic planar lattices under uniaxial compression, part II: Dynamic crushing based on finite element simulation, International Journal of Impact Engineering, 36 (2009) 1231-1241.

[82] S.-Y. He, Y. Zhang, G. Dai, J.-Q. Jiang, Preparation of density-graded aluminum foam, Materials Science and Engineering: A, 618 (2014) 496-499.

[83] J. Zheng, Q. Qin, T.J. Wang, Impact plastic crushing and design of density-graded cellular materials, Mechanics of Materials, 94 (2016) 66-78.

[84] F.R. Jr. Cichocki, K.P. Trumble, J. Rödel, Tailored Porosity Gradients via Colloidal Infiltration of Compression-Molded Sponges, J Am Ceram Soc, 81 (1998) 1661-1664.

[85] A. Ajdari, B.H. Jahromi, J. Papadopoulos, H. Nayeb-Hashemi, A. Vaziri, Hierarchical honeycombs with tailorable properties, International Journal of Solids and Structures, 49 (2012) 1413-1419.

[86] T. Yi, C.Q. Chen, The impact response of clamped sandwich beams with ordinary and hierarchical cellular cores, International Journal of Impact Engineering, 47 (2012) 14-23.

[87] X. Zheng, W. Smith, J Jackson, B. Moran, H. Cui, D. Chen, J. Ye, N. Fang, N. Rodriguez, T. Weisgraber, C.M. Spadaccini, Multiscale metallic metamaterials, Nature Materials, advance online publication (2016).

[88] B. Song, W. Chen, W.-Y. Lu, Compressive mechanical response of a low-density epoxy foam at various strain rates, Journal of Materials Science, 42 (2007) 7502-7507.

[89] C. Tekoğlu, L.J. Gibson, T. Pardoen, P.R. Onck, Size effects in foams: Experiments and modeling, Progress in Materials Science, 56 (2011) 109-138.

[90] E.W. Andrews, G. Gioux, P. Onck, L.J. Gibson, Size effects in ductile cellular solids. Part II: experimental results, International Journal of Mechanical Sciences, 43 (2001) $701-713$.

[91] P.R. Onck, E.W. Andrews, L.J. Gibson, Size effects in ductile cellular solids. Part I: modeling, International Journal of Mechanical Sciences, 43 (2001) 681-699.

[92] M. Vural, G. Ravichandran, Dynamic response and energy dissipation characteristics of balsa wood: experiment and analysis, International Journal of Solids and Structures, 40 (2003) 2147-2170. 
[93] Q.M. Li, R.A.W. Mines, Strain Measures for Rigid Crushable Foam in Uniaxial Compression, Strain, 38 (2002) 132-140.

[94] Q.M. Li, R.A.W. Mines, R.S. Birch, The crush behaviour of Rohacell-51WF structural foam, International Journal of Solids and Structures, 37 (2000) 6321-6341.

[95] E. de Sousa, C.R. Rambo, D. Hotza, A.P.N.d. Oliveira, T. Fey, P. Greil, Microstructure and properties of LZSA glass-ceramic foams, Materials Science and Engineering: A, 476 (2008) 89-97.

[96] Y. Sun, B. Amirrasouli, S.B. Razavi, Q.M. Li, T. Lowe, P.J. Withers, The variation in elastic modulus throughout the compression of foam materials, Acta Materialia, 110(2016) 161-174.

[97] A. Ahmad Zaidi, Q. LI, Investigation on penetration resistance of foamed concrete, Proceedings of the Institution of Civil Engineers. Structures and buildings, 162 (2009) 77-85.

[98] F. Triawan, K. Kishimoto, T. Adachi, K. Inaba, T. Nakamura, T. Hashimura, The elastic behavior of aluminum alloy foam under uniaxial loading and bending conditions, Acta Materialia, 60 (2012) 3084-3093.

[99] E.A. Flores-Johnson, Q.M. Li, R.A.W. Mines, Degradation of Elastic Modulus of Progressively Crushable Foams in uniaxial compression, Journal of Cellular Plastics, 44 (2008) 415-434.

[100] Q.M. Li, I. Magkiriadis, J.J. Harrigan, Compressive Strain at the Onset of Densification of Cellular Solids, Journal of Cellalar Plastics, 42 (2006) 371-392.

[101] P.J. Tan, J.J. Harrigan, S.R. Reid, Inertia effects in uniaxial dynamic compression of a closed cell aluminium alloy foam, Materials Science and Technology, 18 (2002) 480-488.

[102] E.A. Flores-Johnson, Q.M. Li, Indentation into polymeric foams, International Journal of Solids and Structures, 47 (2010) 1987-1995.

[103] M.I. Idris, T. Vodenitcharova, M. Hoffman, Mechanical behaviour and energy absorption of closed-cell aluminium foam panels in uniaxial compression, Materials Science and Engineering: A, 517 (2009) 37-45.

[104] Z. Wang, J. Shen, G. Lu, L. Zhao, Compressive behavior of closed-cell aluminum alloy foams at medium strain rates, Materials Science and Engineering: A, 528 (2011) 2326-2330.

[105] Z. Li, J. Zhang, J. Fan, Z. Wang, L. Zhao, On crushing response of the three-dimensional closed-cell foam based on Voronoi model, Mechanics of Materials, 68 (2014) 85-94. 
[106] Z. Zheng, J. Yu, C. Wang, S. Liao, Y. Liu, Dynamic crushing of cellular materials: A unified framework of plastic shock wave models, International Journal of Impact Engineering, 53 (2013) 29-43.

[107] J. Shen, G. Lu, D. Ruan, Compressive behaviour of closed-cell aluminium foams at high strain rates, Composites Part B: Engineering, 41 (2010) 678-685.

[108] S. Xu, D. Ruan, G. Lu, Strength enhancement of aluminium foams and honeycombs by entrapped air under dynamic loadings, International Journal of Impact Engineering, 74 (2014) $120-125$.

[109] L. Wang, Y. Ding, L. Yang, Experimental investigation on dynamic constitutive behavior of aluminum foams by new inverse methods from wave propagation measurements, International Journal of Impact Engineering, 62 (2013) 48-59.

[110] D.-N. Fang, Y.-L. Li, H. Zhao, On the behaviour characterization of metallic cellular materials under impact loading, Acta Mechanica Sinica, 26 (2010) 837-846.

[111] M. Peroni, G. Solomos, V. Pizzinato, Impact behaviour testing of aluminium foam, International Journal of Impact Engineering, 53 (2013) 74-83.

[112] I. Irausquín, J.L. Pérez-Castellanos, V. Miranda, F. Teixeira-Dias, Evaluation of the effect of the strain rate on the compressive response of a closed-cell aluminium foam using the split Hopkinson pressure bar test, Materials \& Design, 47 (2013) 698-705.

[113] H. Zhao, G. Gary, J.R. Klepaczko, On the use of a viscoelastic split hopkinson pressure bar, International Journal of Impact Engineering, 19 (1997) 319-330.

[114] P. Wang, S. Xu, Z. Li, J. Yang, C. Zhang, H. Zheng, S. Hu, Experimental investigation on the strain-rate effect and inertia effect of closed-cell aluminum foam subjected to dynamic loading, Materials Science and Engineering: A, 620 (2015) 253-261.

[115] R. Montanini, Measurement of strain rate sensitivity of aluminium foams for energy dissipation, International Journal of Mechanical Sciences, 47 (2005) 26-42.

[116] R. Rajendran, A. Moorthi, S. Basu, Numerical simulation of drop weight impact behaviour of closed cell aluminium foam, Materials \& Design, 30 (2009) 2823-2830.

[117] L. Wang, J. Zhu, H. Lai, A New Method Combining Lagrangian Analysis with Hopkinson Pressure Bar Technique, Strain, 47 (2011) 173-182.

[118] B. Koohbor, A. Kidane, W.-Y. Lu, M.A. Sutton, Investigation of the dynamic stress-strain response of compressible polymeric foam using a non-parametric analysis, International Journal of Impact Engineering, 91 (2016) 170-182.

[119] U. Ramamurty, A. Paul, Variability in mechanical properties of a metal foam, Acta Materialia, 52 (2004) 869-876. 
[120] I. Duarte, M. Vesenjak, L. Krstulović-Opara, Variation of quasi-static and dynamic compressive properties in a single aluminium foam block, Materials Science and Engineering: A, 616 (2014) 171-182.

[121] A. Nagy, W.L. Ko, U.S. Lindholm, Mechanical Behavior of Foamed Materials Under Dynamic Compression, Journal of Cellular Plastics, 10 (1974) 127-134.

[122] J. Zhang, N. Kikuchi, V. Li, A. Yee, G. Nusholtz, Constitutive modeling of polymeric foam material subjected to dynamic crash loading, International Journal of Impact Engineering, 21 (1998) 369-386.

[123] J.A. Sherwood, C.C. Frost, Constitutive modeling and simulation of energy absorbing polyurethane foam under impact loading, Polymer Engineering \& Science, 32 (1992) 1138-1146.

[124] S. Ouellet, D. Cronin, M. Worswick, Compressive response of polymeric foams under quasi-static, medium and high strain rate conditions, Polym Test, 25 (2006) 731-743.

[125] E. Kasparek, U. Zencker, R. Scheidemann, H. Volzke, K. Muller, Numerical and experimental studies of polyurethane foam under impact loading, Computational Materials Science, 50 (2011) 1353-1358.

[126] R.P. Merrett, G.S. Langdon, M.D. Theobald, The blast and impact loading of aluminium foam, Materials \& Design, 44 (2013) 311-319.

[127] V.L. Tagarielli, V.S. Deshpande, N.A. Fleck, The high strain rate response of PVC foams and end-grain balsa wood, Composites Part B: Engineering, 39 (2008) 83-91.

[128] C. Park, S.R. Nutt, Strain rate sensitivity and defects in steel foam, Materials Science and Engineering: A, 323 (2002) 358-366.

[129] U. Chakravarty, H. Mahfuz, M. Saha, S. Jeelani, Strain rate effects on sandwich core materials: An experimental and analytical investigation, Acta Materialia, 51 (2003) 1469-1479.

[130] T. Mukai,,H. Kanahashi, T. Miyoshi, M. Mabuchi, T. Nieh, K. Higashi, Experimental study of energy absorption in a close-celled aluminum foam under dynamic loading, Scripta Materialia, 40 (1999) 921-928.

[131] A. Paul, U. Ramamurty, Strain rate sensitivity of a closed-cell aluminum foam, Materials Science and Engineering: A, 281 (2000) 1-7.

[132] S. Ramachandra, P. Sudheer Kumar, U. Ramamurty, Impact energy absorption in an Al foam at low velocities, Scripta Materialia, 49 (2003) 741-745. 
[133] F. Yi, Z. Zhu, F. Zu, S. Hu, P. Yi, Strain rate effects on the compressive property and the energy-absorbing capacity of aluminum alloy foams, Materials Characterization, 47 (2001) 417-422.

[134] L. Peroni, M. Avalle, M. Peroni, The mechanical behaviour of aluminium foam structures in different loading conditions, International Journal of Impact Engineering, 35 (2008) 644-658.

[135] Z.Y. Dou, L.T. Jiang, G.H. Wu, Q. Zhang, Z.Y. Xiu, G.Q. Chen, High strain rate compression of cenosphere-pure aluminum syntactic foams, Scripta Materialia, 57 (2007) 945-948.

[136] I.W. Hall, M. Guden, C.J. Yu, Crushing of aluminum closed cell foams: density and strain rate effects, Scripta Materialia, 43 (2000) 515-521.

[137] S. Xu, J.H. Beynon, D. Ruan, G. Lu, Experimental study of the out-of-plane dynamic compression of hexagonal honeycombs, Composite Structures, 94 (2012) 2326-2336.

[138] S. Xu, J.H. Beynon, D. Ruan, T.X. Yu, Strength enhancement of aluminium honeycombs caused by entrapped air under dynamic out-of-plane compression, International Journal of Impact Engineering, 47 (2012) 1-13.

[139] H. Zhao, G. Gary, Crushing behaviour of aluminium honeycombs under impact loading, International Journal of Impact Engineering, 21 (1998) 827-836.

[140] S. Xu, J. Shen, J. Beynon, D. Ruan, G. Lu, High rate compressive behavior of aluminum foams by modified SHPB technique, in: 21st Australasian Conference on the Mechanics of Structures and Materials,(ACMSM21)-Incorporating Sustainable Practice in Mechanics of Structures and Materials, Crc Press, 2010, pp. 727-732.

[141] T. Mukai, T. Miyoshi, S. Nakano, H. Somekawa, K. Higashi, Compressive response of a closed-cell aluminum foam at high strain rate, Scripta Materialia, 54 (2006) 533-537.

[142] C.M. Cady, G.T. Gray Iii, C. Liu, M.L. Lovato, T. Mukai, Compressive properties of a closed-cell aluminum foam as a function of strain rate and temperature, Materials Science and Engineering: A, 525 (2009) 1-6.

[143] K.A. Dannemann, J. Lankford Jr, High strain rate compression of closed-cell aluminium foams, Materials Science and Engineering: A, 293 (2000) 157-164.

[144] F. Han, H. Cheng, Q. Wang, Z. Li, The strain rate effect of an open cell aluminum foam, Metallurgical and Materials Transactions A, 36 (2005) 645-650.

[145] P.J. Tan, S.R. Reid, J.J. Harrigan, On the dynamic mechanical properties of open-cell metal foams - A re-assessment of the 'simple-shock theory', International Journal of Solids and Structures, 49 (2012) 2744-2753. 
[146] U.K. Chakravarty, An investigation on the dynamic response of polymeric, metallic, and biomaterial foams, Composite Structures, 92 (2010) 2339-2344.

[147] D.D. Radford, V.S. Deshpande, N.A. Fleck, The use of metal foam projectiles to simulate shock loading on a structure, International Journal of Impact Engineering, 31 (2005) 1152-1171.

[148] S.L. Lopatnikov, B.A. Gama, J. Haque, C. Krauthauser, J.W. Gillespie Jr, M. Guden, I.W. Hall, Dynamics of metal foam deformation during Taylor cylinder-Hopkinson bar impact experiment, Composite Structures, 61 (2003) 61-71.

[149] Z. Zheng, C. Wang, J. Yu, S.R. Reid, J.J. Harrigan, Dynamic stress-strain states for metal foams using a 3D cellular model, Journal of the Mechanics and Physics of Solids, 72 (2014) 93-114.

[150] L. Wang, Foundations of stress waves, Elsevier Science Ltd, Amsterdam, 2007.

[151] S.R. Reid, T.Y. Reddy, Experimental investigation of inertia effects in one-dimensional metal ring systems subjected to end impact - I. Fixed-ended systems, International Journal of Impact Engineering, 1 (1983) 85-106.

[152] S.R. Reid, W.W. Bell, R.A. Barr, Structural plastic shock model for one-dimensional ring systems, International Journal of Impact Engineering, 1 (1983) 175-191.

[153] S.R. Reid, C. Peng, Dynamic uniaxial crushing of wood, International Journal of Impact Engineering, 19 (1997) 531-570.

[154] J.J. Harrigan, S.R. Reid, P.J. Tan, T. Yella Reddy, High rate crushing of wood along the grain, International Journal of Mechanical Sciences, 47 (2005) 521-544.

[155] L. Davison, Fundamentals of shock wave propagation in solids, Springer, 2008.

[156] Y. Ding, S. Wang, Z. Zheng, L. Yang, J. Yu, Dynamic crushing of cellular materials: A unique dynamic stress-strain state curve, Mechanics of Materials, 100 (2016) 219-231.

[157] S. Wang, Y. Ding, C. Wang, Z. Zheng, J. Yu, Dynamic material parameters of closed-cell foams under high-velocity impact, International Journal of Impact Engineering, 99 (2017) $111+121$.

[158] S. Gaitanaros, S. Kyriakides, Dynamic crushing of aluminum foams: Part II - Analysis, International Journal of Solids and Structures, 51 (2014) 1646-1661.

[159] S. Gaitanaros, S. Kyriakides, On the effect of relative density on the crushing and energy absorption of open-cell foams under impact, International Journal of Impact Engineering, 82 (2015) 3-13. 
[160] C.E. Morris, Shock-wave equation-of-state studies at Los Alamos, Shock Waves, 1 (1991) 213-222.

[161] E. Zaretsky, Z. Asaf, E. Ran, F. Aizik, Impact response of high density flexible polyurethane foam, International Journal of Impact Engineering, 39 (2012) 1-7.

[162] M.A. Meyers, Dynamic behavior of materials, John Wiley \& Sons, 1994.

[163] L.L. Hu, T.X. Yu, Mechanical behavior of hexagonal honeycombs under low-velocity impact - theory and simulations, International Journal of Solids and Structures, 50 (2013) 3152-3165.

[164] C. Chen, R. Lakes, Dynamic wave dispersion and loss properties of conventional and negative Poisson's ratio polymeric cellular materials, Cellular Polymers, 8 (1989) 343-369.

[165] M.A. Kader, M.A. Islam, P.J. Hazell, J.P. Escobedo, M. Saadatfar,/A.D. Brown, G.J. Appleby-Thomas, Modelling and characterization of cell collapse in aluminium foams during dynamic loading, International Journal of Impact Engineering, 96 (2016) 78-88.

[166] H. Toda, M. Takata, T. Ohgaki, M. Kobayashi, T. Kobayashi, K. Uesugi, K. Makii, Y. Aruga, 3-D Image-Based Mechanical Simulation of Aluminium Foams: Effects of Internal Microstructure, Advanced Engineering Materials, 8 (2006) 459-467.

[167] Q.M. Li, S.R. Reid, About one-dimensional shock propagation in a cellular material, International Journal of Impact Engineering, 32 (2006) 1898-1906.

[168] E. Andrews, W. Sanders, L.J. Gibson, Compressive and tensile behaviour of aluminum foams, Materials Science and Engineering A, 270 (1999) 113-124.

[169] Z. Zheng, Y. Lia, J. Yu, S.R. Reid, Dynamic crushing of cellular materials: Continuum-based wave models for the transitional and shock modes, International Journal of Impact Engineering, 42 (2012) 66-79.

[170] L. Li, P. Xue, G. Luo, A numerical study on deformation mode and strength enhancement of metal foam under dynamic loading, Materials \& Design, 110 (2016) 72-79.

[171] J.J. Harrigan, S.R. Reid, A. Seyed Yaghoubi, The correct analysis of shocks in a cellular material, International Journal of Impact Engineering, 37 (2010) 918-927.

[172] D. Karagiozova, G.S. Langdon, G.N. Nurick, Propagation of compaction waves in metal foams exhibiting strain hardening, International Journal of Solids and Structures, 49 (2012) 2763-2777.

[173] S.A. McDonald, P.M. Mummery, G. Johnson, P.J. Withers, Characterization of the three-dimensional structure of a metallic foam during compressive deformation, Journal of Microscopy, 223 (2006) 150-158. 
[174] A. Hönig, W.J. Stronge, In-plane dynamic crushing of honeycomb. Part I: crush band initiation and wave trapping, International Journal of Mechanical Sciences, 44 (2002) 1665-1696.

[175] A. Hönig, W.J. Stronge, In-plane dynamic crushing of honeycomb. Part II: application to impact, International Journal of Mechanical Sciences, 44 (2002) 1697-1714.

[176] H.J. Frost, M.F. Ashby, Deformation mechanism maps: the plasticity and creep of metals and ceramics, (1982).

[177] N. Jones, Structural impact, Cambridge university press, 2011.

[178] S. Walley, J. Field, Strain rate sensitivity of polymers in compression from low to high rates, DYMAT J, 1 (1994) 211-227.

[179] C.H. Karnes, E.A. Ripperger, Strain rate effects in cold worked high-purity Aluminium, Journal of the Mechanics and Physics of Solids, 14 (1966) 75-88.

[180] A.S. Khan, S. Huang, Experimental and theoretical study of mechanical behavior of 1100 aluminum in the strain rate range $10-5-104 \mathrm{~s}-1$, International Journal of Plasticity, 8 (1992) 397-424.

[181] K. Kitazono, Y. Takiguchi, Strain rate sensitivity and energy absorption of Zn-22Al foams, Scripta Materialia, 55 (2006) 501-504.

[182] T. Miyoshi, M. Itoh, S. Akiyama, A. Kitahara, ALPORAS aluminum foam: production process, properties, and applications, Advanced Engineering Materials, 2 (2000) 179-183.

[183] M.A. Islam, M.A. Kader, P.J. Hazell, A.D. Brown, M. Saadatfar, M.Z. Quadir, J.P. Escobedo, Investigation of microstructural and mechanical properties of cell walls of closed-cell aluminium alloy foams, Materials Science and Engineering: A, 666 (2016) 245-256.

[184] J. Zhou, S. Allameh, W.O. Soboyejo, Microscale testing of the strut in open cell aluminum foams, Journal of Materials Science, 40 (2005) 429-439.

[185] A.E. Markaki, T.W. Clyne, The effect of cell wall microstructure on the deformation and fracture of aluminium-based foams, Acta Materialia, 49 (2001) 1677-1686.

[186] G.W. Ma, X.J. Wang, Q.M. Li, Modeling Strain Rate Effect of Heterogeneous Materials Using SPH Method, Rock Mechanics and Rock Engineering, 43 (2010) 763-776.

[187] H. Toda, T. Kobayashi, M. Niinomi, T. Ohgaki, M. Kobayashi, N. Kuroda, T. Akahori, K. Uesugi, K. Makii, Y. Aruga, Quantitative assessment of microstructure and its effects on compression behavior of aluminum foams via high-resolution synchrotron X-ray tomography, Metallurgical and Materials Transactions A, 37 (2006) 1211-1219. 
[188] B. Koohbor, S. Ravindran, A. Kidane, Effects of cell-wall instability and local failure on the response of closed-cell polymeric foams subjected to dynamic loading, Mechanics of Materials, (2017).

[189] E. Maire, P.J. Withers, Quantitative X-ray tomography, International Materials Reviews, 59 (2014) 1-43.

[190] M. Vesenjak, C. Veyhl, T. Fiedler, Analysis of anisotropy and strain rate sensitivity of open-cell metal foam, Materials Science and Engineering: A, 541 (2012) 105-109.

[191] A.H. Benouali, L. Froyen, T. Dillard, S. Forest, F. N'guyen, Investigation on the influence of cell shape anisotropy on the mechanical performance of closed cell aluminium foams using micro-computed tomography, Journal of Materials Seience, 40 (2005) $5801-5811$.

[192] P. Wang, S. Xu, Z. Li, J. Yang, H. Zheng, S. Hu, Temperature effects on the mechanical behavior of aluminum foam under dynamic loading, Materials Science and Engineering: A, 599 (2014) 174-179.

[193] I. Jeon, K. Katou, T. Sonoda, T. Asahina, K.-J. Kang, Cell wall mechanical properties of closed-cell Al foam, Mechanics of Materials, 41 (2009) 60-73.

[194] A. Hönig, W.J. Stronge, Dynamic buckling of an imperfect elastic, visco-plastic plate, International Journal of Impact Engineering, 24 (2000) 907-923.

[195] X.Y. Su, T.X. Yu, S.R. Reid, Inertia-sensitive impact energy-absorbing structures part I: Effects of inertia and elasticity, International Journal of Impact Engineering, 16 (1995) 651-672.

[196] X.Y. Su, T.X. Yu, S.R. Reid, Inertia-sensitive impact energy-absorbing structures part II: Effect of strain rate, International Journal of Impact Engineering, 16 (1995) 673-689.

[197] T.G. Zhang, T.X. Yu, A note on a 'velocity sensitive' energy-absorbing structure, International Journal of Impact Engineering, 8 (1989) 43-51.

[198] Z.Y. Gad, T.X. Yu, G. Lu, A study on type II structures. Part I: a modified one-dimensional mass-spring model, International Journal of Impact Engineering, 31 (2005) 895-910.

[199] Z. Zou, P.J. Tan, S.R. Reid, S. Li, J.J. Harrigan, Dynamic crushing of a one-dimensional chain of type II structures, International Journal of Impact Engineering, 34 (2007) 303-328.

[200] J.C. Maxwell, On the calculation of the equilibrium and stiffness of frames, Philosophical Magazine, 27 (1864) 294-299. 
[201] S.D. Papka, S. Kyriakides, Experiments and full-scale numerical simulations of in-plane crushing of a honeycomb, Acta Materialia, 46 (1998) 2765-2776.

[202] A. Wilbert, W.Y. Jang, S. Kyriakides, J.F. Floccari, Buckling and progressive crushing of laterally loaded honeycomb, International Journal of Solids and Structures, 48 (2011) 803-816.

[203] D. Mohr, M. Doyoyo, Nucleation and propagation of plastic collapse bands in aluminum honeycomb, Journal of Applied Physics, 94 (2003) 2262-2270.

[204] A. Da Silva, S. Kyriakides, Compressive response and failure of balsa wood, International Journal of Solids and Structures, 44 (2007) 8685-8717.

[205] J. Zhou, P. Shrotriya, W.O. Soboyejo, Mechanisms and mechanics of compressive deformation in open-cell Al foams, Mechanics of Materials, 36 (2004) 781-797.

[206] W.-Y. Jang, S. Kyriakides, On the crushing of aluminum open-cell foams: Part I. Experiments, International Journal of Solids and Structures, 46 (2009) 617-634.

[207] W.-Y. Jang, S. Kyriakides, On the crushing of aluminum open-cell foams: Part II analysis, International Journal of Solids and Structures, 46 (2009) 635-650.

[208] P.J. Tan, S.R. Reid, J.J. Harrigan, Discussion: 'The Resistance of Clamped Sandwich Beams to Shock Loading" (Fleck, N. A., and Deshpande, V. S., 2004, ASME J. Appl. Mech., [bold 71], pp. 386--401), Journal of Applied Mechanics, 72 (2005) 978-979.

[209] N.A. Fleck, V.S. Deshpande, Closure to 'Discussion of 'The Resistance of Clamped Sandwich Beams to Shock Loading' " (2005, ASME J. Appl. Mech., [bold 72], pp. 978--979), Journal of Applied Mechanics, 72 (2005) 980.

[210] S. Pattofatto, I. Elnasri, H. Zhao, H. Tsitsiris, F. Hild, Y. Girard, Shock enhancement of cellular structures under impact loading: Part II analysis, Journal of the Mechanics and Physics of Solids, 55 (2007) 2672-2686.

[211] R. Edwin Raj, V. Parameswaran, B.S.S. Daniel, Comparison of quasi-static and dynamic compression behavior of closed-cell aluminum foam, Materials Science and Engineering: A, 526 (2009) 11-15.

[212] K. Kitazono, E. Sato, K. Kuribayashi, Application of mean-field approximation to elastic-plastic behavior for closed-cell metal foams, Acta Materialia, 51 (2003) 4823-4836.

[213] A. Öchsner, G. Mishuris, Modelling of the multiaxial elasto-plastic behaviour of porous metals with internal gas pressure, Finite Elements in Analysis and Design, 45 (2009) 104-112.

[214] W. Zhang, Z. Xu, T.J. Wang, X. Chen, Effect of inner gas pressure on the elastoplastic behavior of porous materials: A second-order moment micromechanics model, International Journal of Plasticity, 25 (2009) 1231-1252. 
[215] Z.M. Xu, W.X. Zhang, T.J. Wang, Deformation of closed-cell foams incorporating the effect of inner gas pressure, International Journal of Applied Mechanics, 02 (2010) 489-513.

[216] M.W. Schraad, F.H. Harlow, A multi-field approach to modeling the dynamic response of cellular materials, International Journal of Mechanical Sciences, 48 (2006) 85-106.

[217] X.W. Zhang, T.X. Yu, Energy absorption of pressurized thin-walled circular tubes under axial crushing, International Journal of Mechanical Sciences, 51 (2009) 335-349.

[218] O. Sadot, O. Ram, I. Anteby, S. Gruntman, G. Ben-Dor, The trapped gas effect on the dynamic compressive strength of light aluminum foams, Materials Science and Engineering: A, 659 (2016) 278-286.

[219] B. Banerjee, A. Bhawalkar, Numerical simulation of the dynamic compression of a 6061-T6 aluminum metallic foam, in, 7th World Conference on Computational Mechanics, July 2006.

[220] Q. Fang, J. Zhang, Y. Zhang, Z. Gong, H. Wu, A 3D mesoscopic model for the closed-cell metallic foams subjected to static and dynamic loadings, International Journal of Impact Engineering, 82 (2015) 103-112.

[221] Y. Alvandi-Tabrizi, D.A. Whisler, H. Kim, A. Rabiei, High strain rate behavior of composite metal foams, Materials Science and Engineering: A, 631 (2015) 248-257.

[222] W.J. Stronge, V.P.W. Shim, Microdynamics of Crushing in Cellular Solids, Journal of Engineering Materials and Technology, 110 (1988) 185-190.

[223] V.P.W. Shim, B.Y. Tay, W.J. Stronge, Dynamic Crushing of Strain-Softening Cellular Structures---A One-Dimensional Analysis, Journal of Engineering Materials and Technology, 112 (1990) 398-405.

[224] V.P.W. Shim, K.Y. Yáp, W.J. Stronge, Effects of nonhomogeneity, cell damage and strain-rate on impact crushing of a strain-softening cellular chain, International Journal of Impact Engineering, 12 (1992) 585-602.

[225] Z. Wang, Y. Zhang, H. Ren, L. Zhao, A study on compressive shock wave propagation in metallic foams, Science China Physics, Mechanics and Astronomy, 53 (2010) 279-287.

[226] T. Daxner, H.J. Böhm, F.G. Rammerstorfer, Mesoscopic simulation of inhomogeneous metallic foams with respect to energy absorption, Computational Materials Science, 16 (1999) 61-69.

[227] A.P. Roberts, E.J. Garboczi, Elastic moduli of model random three-dimensional closed-cell cellular solids, Acta Materialia, 49 (2001) 189-197. 
[228] L. Li, P. Xue, Y. Chen, H.S.U. Butt, Insight into cell size effects on quasi-static and dynamic compressive properties of 3D foams, Materials Science and Engineering: A, 636 (2015) 60-69.

[229] L. Hu, F. You, T. Yu, Analyses on the dynamic strength of honeycombs under the y-directional crushing, Materials \& Design, 53 (2014) 293-301.

[230] L.L. Hu, T.X. Yu, Dynamic crushing strength of hexagonal honeycombs, International Journal of Impact Engineering, 37 (2010) 467-474.

[231] L.L. Hu, L. Hu, D.Y. Cai, Undulation of the honeycombs' plateau stress under impact and the dynamic enhancement-Theoretical analysis, Materials Science and Engineering: A, 598 (2014) 190-196.

[232] T. Daxner, Finite Element Modeling of Cellular Materials, Cellulár and Porous Materials in Structures and Processes, 521 (2010) 47-106.

[233] C. Petit, S. Meille, E. Maire, Cellular solids studied by x-ray tomography and finite element modeling - a review, Journal of Materials Research, 28 (2013) 2191-2201.

[234] E. Maire, X-ray tomography applied to the characterization of highly porous materials, Annual Review of Materials Research, 42 (2012) 163-178.

[235] O. Caty, E. Maire, S. Youssef, R. Bouchet, Modeling the properties of closed-cell cellular materials from tomography images using finite shell elements, Acta Materialia, 56 (2008) 5524-5534.

[236] S.G. Bardenhagen, A.D. Brydon, J.E. Guilkey, Insight into the physics of foam densification via numerical simulation, Journal of the Mechanics and Physics of Solids, 53 (2005) 597-617.

[237] A.D. Brydon, S.G. Bardenhagen, E.A. Miller, G.T. Seidler, Simulation of the densification of real open-celled foam microstructures, Journal of the Mechanics and Physics of Solids, 53 (2005) 2638-2660.

[238] C. Veyhl, I.V. Belova, G.E. Murch, T. Fiedler, Finite element analysis of the mechanical properties of cellular aluminium based on micro-computed tomography, Materials Science and Engineering: A, 528 (2011) 4550-4555.

[239] Y. Chen, R. Das, M. Battley, Finite element analysis of the compressive and shear responses of structural foams using computed tomography, Composite Structures, 159 (2017) 784-799.

[240] Y.L. Sun, T. Lowe, S.A. McDonald, Q.M. Li, P.J. Withers, In situ investigation and image-based modelling of aluminium foam compression using micro X-Ray computed tomography, in: Visual Computing: Scientific Visualization and Imaging Systems, Springer, 2014, pp. 189-197. 
[241] Y. Hangai, R. Yamaguchi, S. Takahashi, T. Utsunomiya, O. Kuwazuru, N. Yoshikawa, Deformation Behavior Estimation of Aluminum Foam by X-ray CT Image-based Finite Element Analysis, Metallurgical and Materials Transactions A, 44 (2013) 1880-1886.

[242] Y. Sun, Q. Li, P. Withers, Strain-rate sensitivity of foam materials: A numerical study using 3D image-based finite element model, in: EPJ Web of Conferences, EDP Sciences, 2015, pp. 04022.

[243] C. Veyhl, T. Fiedler, U. Jehring, O. Andersen, T. Bernthaler, I.V. Belova, G.E. Murch, On the mechanical properties of sintered metallic fibre structures, Materials Science and Engineering: A, 562 (2013) 83-88.

[244] D. Sun, W. Zhang, Y. Zhao, G. Li, Y. Xing, G. Gong, In-plane crushing and energy absorption performance of multi-layer regularly arranged circular honeycombs, Composite Structures, 96 (2013) 726-735.

[245] Y. Song, Z. Wang, L. Zhao, J. Luo, Dynamic crushing behavior of 3D closed-cell foams based on Voronoi random model, Materials \& Design, 31 (2010) 4281-4289.

[246] A.G. Hanssen, O.S. Hopperstad, M. Langseth, H. Ilstad, Validation of constitutive models applicable to aluminium foams, International Journal of Mechanical Sciences, 44 (2002) 359-406.

[247] D. Ruan, G. Lu, F.L. Chen, E. Siores, Compressive behaviour of aluminium foams at low and medium strain rates, Composite Structures, 57 (2002) 331-336.

[248] Simulia, ABAQUS Analysis User's Manual, ABAQUS v6.12 Documentation, (Dassault Systémes, V6.12).

[249] R. Darvizeh, K. Davey, A transport approach for analysis of shock waves in cellular materials, International Journal of Impact Engineering, 82 (2015) 59-73.

[250] G. Lu, T. Yu, Energy absorption of structures and materials, Woodhead Publishing Limited, 2003.

[251] N.A. Fleck, V.S. Deshpande, The Resistance of Clamped Sandwich Beams to Shock Loading, Journal of Applied Mechanics, 71 (2004) 386-401.

[252] H. Ousji, B. Belkassem, M.A. Louar, B. Reymen, J. Martino, D. Lecompte, L. Pyl, J. Vantomme, Air-blast response of sacrificial cladding using low density foams: Experimental and analytical approach, International Journal of Mechanical Sciences, 128 (2017) 459-474.

[253] Y. Ding, S. Wang, K. Zhao, Z. Zheng, L. Yang, J. Yu, Blast Alleviation of Cellular Sacrificial Cladding: A Nonlinear Plastic Shock Model, International Journal of Applied Mechanics, 08 (2016) 1650057. 
[254] S.L. Lopatnikov, B.A. Gama, M.J. Haque, C. Krauthauser, J.W. Gillespie Jr, High-velocity plate impact of metal foams, International Journal of Impact Engineering, 30 (2004) 421-445.

[255] D. Karagiozova, G.S. Langdon, G.N. Nurick, Compaction of metal foam subjected to an impact by a low-density deformable projectile, International Journal of Impact Engineering, 62 (2013) 196-209.

[256] G.W. Ma, Z.Q. Ye, Analysis of foam claddings for blast alleviation, International Journal of Impact Engineering, 34 (2007) 60-70.

[257] G.W. Ma, Z.Q. Ye, Energy absorption of double-layer foam cladding for blast alleviation, International Journal of Impact Engineering, 34 (2007) 329-347.

[258] M. Aleyaasin, J.J. Harrigan, S.R. Reid, Air-blast response of cellular material with a face plate: An analytical-numerical approach, International Journal of Mechanical Sciences, 91 (2015) 64-70.

[259] H. Zhou, Z. Zhao, G. Ma, Mitigating Ground Shocks with Cellular Solids, Journal of Engineering Mechanics, 139 (2013) 1362-1371.

[260] D. Karagiozova, G.S. Langdon, G.N. Nurick, Blast attenuation in Cymat foam core sacrificial claddings, International Journal of Mechanical Sciences, 52 (2010) 758-776.

[261] D. Karagiozova, M. Alves, Compaction of a double-layered metal foam block impacting a rigid wall, International Journal of Solids and Structures, 51 (2014) 2424-2438.

[262] D. Karagiozova, M. Alves, Primary and Reflected Compaction Waves in a Foam Rod Due to an Axial Impact by a Small Mass, Latin American Journal of Solids and Structures, 12 (2015) 905-924.

[263] H. Askes, E.C. Aifantis, Gradient elasticity in statics and dynamics: An overview of formulations, length scale identification procedures, finite element implementations and new results, International Journal of Solids and Structures, 48 (2011) 1962-1990.

[264] A.R. Hadjesfandiari, G.F. Dargush, Couple stress theory for solids, International Journal of Solids and Structures, 48 (2011) 2496-2510.

[265] R.S. Kumar, D.L. McDowell, Generalized continuum modeling of 2-D periodic cellular solids, International Journal of Solids and Structures, 41 (2004) 7399-7422.

[266] A. Berezovski, J. Engelbrecht, A. Salupere, K. Tamm, T. Peets, M. Berezovski, Dispersive waves in microstructured solids, International Journal of Solids and Structures, 50 (2013). 
[267] L.J. Gibson, M.F. Ashby, J. Zhang, T.C. Triantafillou, Failure surfaces for cellular materials under multiaxial loads-I.Modelling, International Journal of Mechanical Sciences, 31 (1989) 635-663.

[268] S. Abrate, Criteria for yielding or failure of cellular materials, Journal of Sandwich Structures and Materials, 10 (2008) 5-51.

[269] D.C. Drucker, W. Prager, Soil mechanics and plastic analysis or limit design, Q Appl Math, 10 (1952) 157-165.

[270] A. Maji, H. Schreyer, S. Donald, Q. Zuo, D. Satpathi, Mechanical Properties of Polyurethane-Foam Impact Limiters, Journal of engineering mechanics, 121 (1995) 528-540.

[271] M. Doyoyo, T. Wierzbicki, Experimental studies on the yield behavior of ductile and brittle aluminum foams, International Journal of Plasticity, 19 (2003) 1195-1214.

[272] J. Zhang, Z. Lin, A. Wong, N. Kikuchi, V.C. Li, A. Yee, G. Nusholtz, Constitutive modeling and material characterization of polymeric foams, Journal of Engineering Materials and Technology, 119 (1997) 284.

[273] R.E. Miller, A continuum plasticity model for the constitutive and indentation behaviour of foamed metals, International Journal of Mechanical Sciences, 42 (2000) 729-754.

[274] Z. Zhou, Z. Wang, L. Zhao, X. Shu, Loading rate effect on yield surface of aluminum alloy foams, Materials Science and Engineering: A, 543 (2012) 193-199.

[275] T.C. Triantafillou, J. Zhang, T.L. Shercliff, L.J. Gibson, M.F. Ashby, Failure surfaces for cellular materials under multiaxial loads-II. Comparison of models with experiment, International Journal of Mechanical Sciences, 31 (1989) 665-678.

[276] J. Hallquist, ANSYS/LS-DYNA Theoretical Manual [M], Livermore Software Technology Corporation, USA, (2005).

[277] Q.H. Qin, TJ. Wang, An analytical solution for the large deflections of a slender sandwich beam/ with a metallic foam core under transverse loading by a flat punch, Composite Structures, 88 (2009) 509-518.

[278] Q.H. Qin, T.J. Wang, S.Z. Zhao, Large deflections of metallic sandwich and monolithic beams under locally impulsive loading, International Journal of Mechanical Sciences, 51 (2009) $752-773$.

[279] Q.H. Qin, T.J. Wang, A theoretical analysis of the dynamic response of metallic sandwich beam under impulsive loading, European Journal of Mechanics - A/Solids, 28 (2009) 1014-1025. 
[280] Q.H. Qin, T.J. Wang, Low-velocity heavy-mass impact response of slender metal foam core sandwich beam, Composite Structures, 93 (2011) 1526-1537.

[281] I. Ivañez, C. Santiuste, S. Sanchez-Saez, FEM analysis of dynamic flexural behaviour of composite sandwich beams with foam core, Composite Structures, 92 (2010) 2285-2291.

[282] A. Rajaneesh, I. Sridhar, S. Rajendran, Impact modeling of foam cored sandwich plates with ductile or brittle faceplates, Composite Structures, 94 (2012) 1745-1754.

[283] J. Zhang, Q. Qin, C. Xiang, Z. Wang, T.J. Wang, A theoretical study of low-velocity impact of geometrically asymmetric sandwich beams, International Journal of Impact Engineering, 96 (2016) 35-49.

[284] J. Zhang, Q. Qin, Z. Wang, W. Ai, T.J. Wang, A theoretical study of plastic analysis of fully clamped geometrical asymmetric sandwich beams with a metal foam core, International Journal of Mechanical Sciences, 99 (2015) 98-111.

[285] J. Zhang, Q. Qin, C. Xiang, T.J. Wang, Dynamic response of slender multilayer sandwich beams with metal foam cores subjected to low-velocity impact, Composite Structures, 153 (2016) 614-623.

[286] R. Rajendran, K. Prem Sai, B. Chandrasekar, A. Gokhale, S. Basu, Preliminary investigation of aluminium foam as an energy absorber for nuclear transportation cask, Materials \& Design, 29 (2008) 1732-1739.

[287] Q. Qin, T.J. Wang, Low-velocity impact response of fully clamped metal foam core sandwich beam incorporating local denting effect, Composite Structures, 96 (2013) 346-356.

[288] J. Wang, A.M. Waas, H. Wang, Experimental and numerical study on the low-velocity impact behavior of foam-core sandwich panels, Composite Structures, 96 (2013) 298-311.

[289] J. Chung, A.M. Wáas, Compressive response of circular cell polycarbonate honeycombs under inplane biaxial static and dynamic loading. Part I: experiments, International Journal of Impact Engineering, 27 (2002) 729-754.

[290] J. Chung, A.M. Waas, Compressive response of circular cell polycarbonate honeycombs under inplane biaxial static and dynamic loading-Part II: simulations, International Journal of Impact Engineering, 27 (2002) 1015-1047.

[291] B. Hou, A. Ono, S. Abdennadher, S. Pattofatto, Y.L. Li, H. Zhao, Impact behavior of honeycombs under combined shear-compression. Part I: Experiments, International Journal of Solids and Structures, 48 (2011) 687-697.

[292] B. Hou, S. Pattofatto, Y.L. Li, H. Zhao, Impact behavior of honeycombs under combined shear-compression. Part II: Analysis, International Journal of Solids and Structures, 48 (2011) 698-705. 
[293] H. Zhou, X. Wang, Z. Zhao, High velocity impact mitigation with gradient cellular solids, Composites Part B: Engineering, 85 (2016) 93-101.

[294] H. Zhou, Y. Wang, X. Wang, Z. Zhao, G. Ma, Energy absorption of graded foam subjected to blast: A theoretical approach, Materials \& Design, 84 (2015) 351-358.

[295] J. Marx, A. Rabiei, Overview of Composite Metal Foams and Their Properties and Performance, Advanced Engineering Materials, (2017) 1600776-n/a.

[296] B.G. Compton, J.A. Lewis, 3D-Printing of Lightweight Cellular Composites, Adv Mater, 26 (2014) 5930-5935.

[297] K.C. Cheung, N. Gershenfeld, Reversibly Assembled Cellular Composite Materials, Science, 341 (2013) 1219-1221. 